FLOODS IN THE NISHNABOTNA RIVER BASIN, IOWA

By David A. Eash and Albert J. Heinitz

U.S. GEOLOGICAL SURVEY

Open-File Report 91-171

Prepared in cooperation with the

Highway Research Advisory Board, Highway Division,

Iowa Department of Transportation

Research Project HR-140

Iowa City, Iowa

1991 


\section{U.S. DEPARTMENT OF THE INTERIOR}

\section{MANUEL LUJAN, JR., Secretary}

U.S. GEOLOGICAL SURVEY

Dallas L. Peck, Director

For additional information write to:

\section{U.S. Geological Survey}

Water Resources Division

Rm. 269, Federal Building

400 South Clinton Street

Iowa City, IA 52244
Copies of this report can be purchased from:

Books and Open-File Reports

U.S. Geological Survey

Federal Center

Box 25425

Denver, CO 80225 


\section{CONTENTS}

\section{Page}

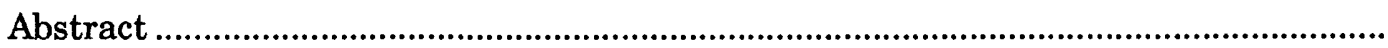



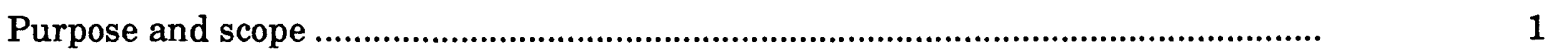

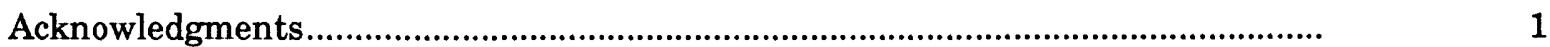

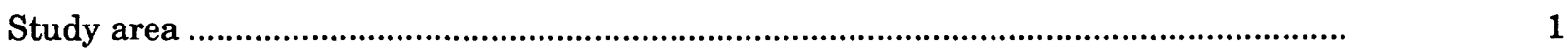

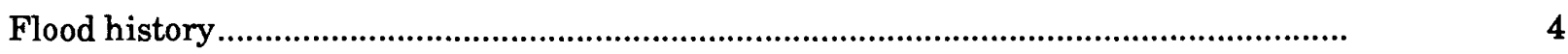

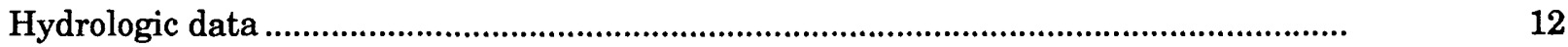

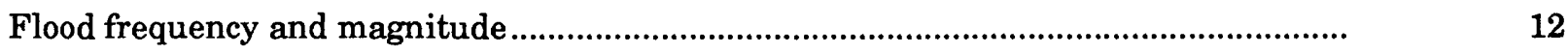

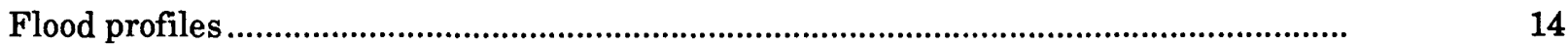








\section{ILLUSTRATIONS}

Figure 1. Map showing location of active and discontinued gaging stations in the Nishnabotna River basin

2. Map showing location of hydrologic regions and miscellaneous measurement sites in the Nishnabotna River basin...

3-28. Graphs showing:

3. Annual peak discharges for period of record for West Nishnabotna River at Hancock gaging station

4. Annual peak discharges for period of record for Indian Creek near Emerson crest-stage gaging station

5. Annual peak discharges for period of record for West Nishnabotna River at Randolph gaging station

6. Annual peak discharges for period of record for East Nishnabotna River near Atlantic gaging station

7. Annual peak discharges for period of record for East Nishnabotna River at Red Oak gaging station

8. Annual peak discharges for period of record for Nishnabotna River above Hamburg gaging station

9. Relation of maximum discharges and regional, 100-year flood discharges with drainage area for active and discontinued gaging stations, and for miscellaneous measurement sites in the Nishnabotna River basin

10. Relation of Bulletin 17B and region 2, 100-year flood discharges with drainage area for streams in the Nishnabotna River basin

11-19. Profiles of West Nishnabotna River

20-27. Profiles of East Nishnabotna River

28. Profile of Indian Creek 


\section{TABLES}

Page

Table 1. Selected flood-peak discharges at active and discontinued gaging stations in the Nishnabotna River basin ........................................................................

2. Flood-peak discharges at miscellaneous, indirect measurement sites in the Nishnabotna River basin.

3. Peak stages and discharges for gaging stations, Nishnabotna River basin, Iowa

4. Discharge and frequency of flood flows for active and discontinued gaging stations in the Nishnabotna River basin..

5. Temporary bench marks, Nishnabotna River basin, Iowa 


\section{CONVERSION FACTORS AND VERTICAL DATUM}

Multiply

inch (in)

foot (ft)

mile (mi)

square foot $\left(\mathrm{ft}^{2}\right)$

acre

acre

square mile $\left(\mathrm{mi}^{2}\right)$

cubic foot per second $\left(\mathrm{ft}^{3} / \mathrm{s}\right)$

cubic foot per second per

square mile $\left[\left(\mathrm{ft}^{3} / \mathrm{s}\right) / \mathrm{mi}^{2}\right]$
$\underline{B y}$

Length

25.4

0.3048

1.609

$\underline{\text { Area }}$

0.09290
4,047
0.4047
2.590

Volume

0.02832

0.01093

$\underline{\text { Mass }}$

ton, short $(2,000 \mathrm{lb})$

ton per acre

0.9072

2.242 $\underline{\text { To obtain }}$

millimeter

meter

kilometer

square meter

square meter

square hectometer

square kilometer

cubic meter per second

cubic meter per second per square

kilometer

megagram

Sea Level: In this report, "sea level" refers to the National Geodetic Vertical Datum of 1929 -a geodetic datum derived from a general adjustment of the first-order level nets of the United States and Canada, formerly called Sea Level Datum of 1929. 


\title{
FLOODS IN THE NISHNABOTNA RIVER BASIN, IOWA
}

\author{
By David A. Eash and Albert J. Heinitz
}

\begin{abstract}
Flood-elevation profiles and flood-peak discharges for floods during 1972, 1982, and 1987 in the Nishnabotna River basin are given in this report. The profiles are for the 1972 flood on the West and East Nishnabotna Rivers, the 1982 flood on Indian Creek, and the 1987 flood on the lower West Nishnabotna River. A flood history describes rainfall conditions and reported damages for floods occurring during 1947, 1958, 1972, 1982, and 1987. Discharge for the 1982 flood on Indian Creek is 1.1 times larger than the 100 -year recurrence interval discharge.
\end{abstract}

\section{INTRODUCTION}

Evaluation of flood hazards, and the planning, design, and operation of various structures on flood plains require information about floods. Flood reports supply specific information for selected floods, and are used by planners and engineers to evaluate the magnitude and frequency of floods in a river basin.

\section{Purpose and Scope}

This report provides information on flood stages and discharges, flood magnitude and frequency, and bench-mark data for the Nishnabotna River basin. It also presents flood-elevation profiles for the 1972, 1982, and 1987 floods. The 1972 profiles are for 107 miles on the West Nishnabotna River, 84 miles on the East Nishnabotna River, and 4 miles on the Nishnabotna River. The 1982 profile is for 9 miles on Indian Creek. The 1987 profiles are for 47 miles on the lower West Nishnabotna River and 4 miles on the Nishnabotna River. A flood history describes rainfall conditions and reported damages for floods occurring during 1947, 1958, 1972, 1982, and 1987.

\section{Acknowledgments}

This report is the thirteenth in a series prepared in cooperation with the Highway Research Advisory Board, Highway Division, Iowa Department of Transportation. Various Federal, State and local agencies cooperated in the collection of the streamflow records used in this report, acknowledgment of which is contained in the annual streamflow reports of the U.S. Geological Survey (USGS).

\section{STUDY AREA}

The Nishnabotna River basin is located in southwestern Iowa and drains into the Missouri River 10 miles south of the Iowa-Missouri border (figs. 1 and 2). The basin covers 2,819 square miles in Iowa and includes parts of 12 counties. The two major tributaries of the Nishnabotna River are the West Nishnabotna River and the East Nishnabotna River, with drainage areas of 1,649 and 1,148 square miles, respectively. The West and East Nishnabotna Rivers follow parallel, southerly courses for about 100 miles before joining at their confluence, 4 miles upstream of Hamburg, to form the Nishnabotna River.

The topography of the Nishnabotna River basin is characterized by broad, rolling uplands and wide valleys; an indication that the basin has reached a stage of geologic maturity. The soil cover of the basin generally consists of a mantle of loess deposited during the interglacial periods and following the 


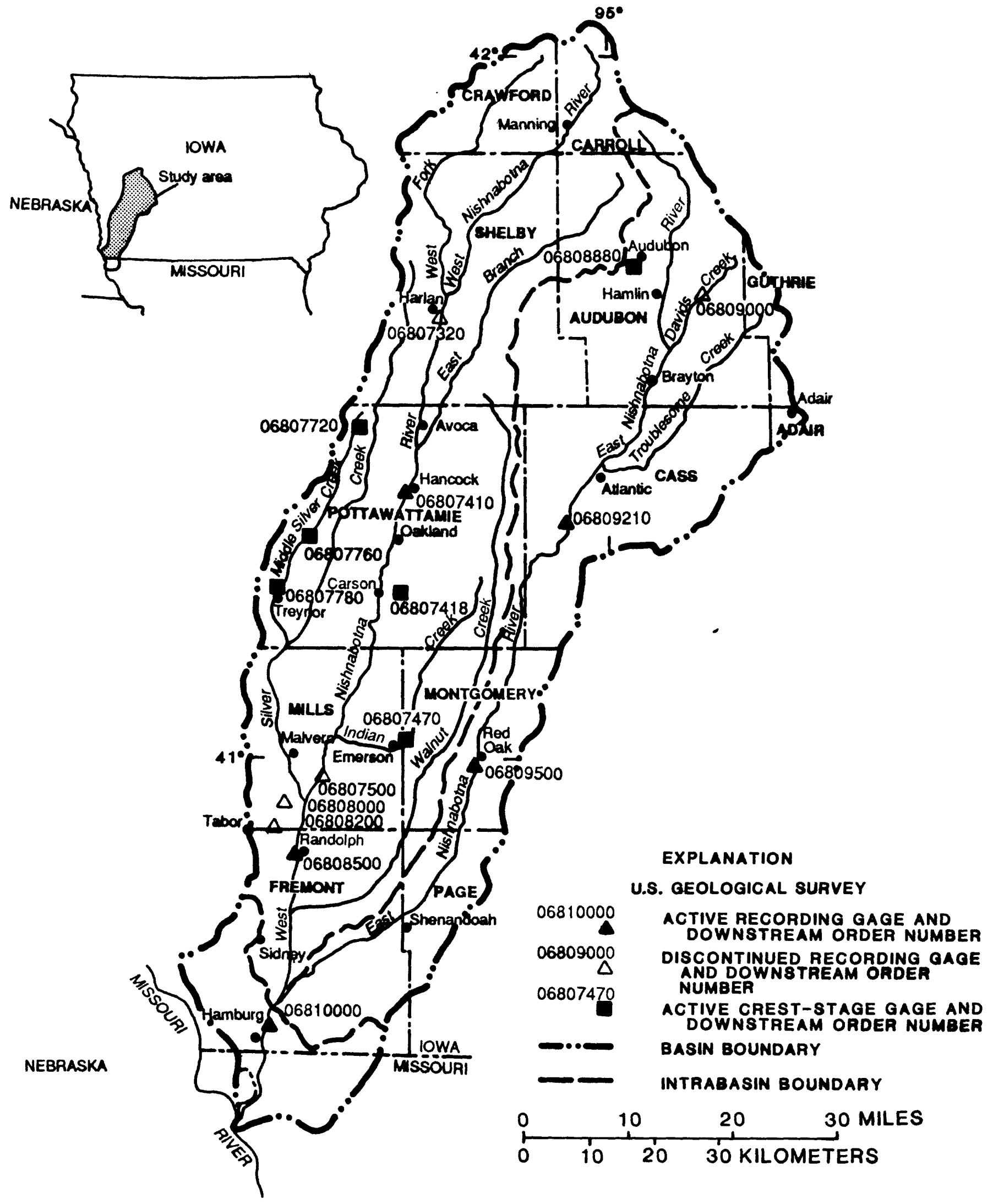

Figure 1.--Location of active and discontinued gaging stations in the Nishnabotna River basin. 




Figure 2.--Location of hydrologic regions and miscellaneous measurement sites in the Nishnabotna River basin. 
advance of ice sheets in Iowa. The loess is almost 100 feet thick in places near the western edge of the basin; it decreases in thickness from west to east until it is only 3 to 8 feet thick on the ridges in the northeast part of the basin (Iowa Natural Resources Council, 1955, p. 3).

Before the turn of the century, streams draining the Nishnabotna River basin were naturally sluggish and meandering, but extensive channel straightening during the early 1900 's altered the streamflow characteristics of the basin. Channel straightening occurred in about 90 percent of the lower 100 miles of the West Nishnabotna River, and in about 75 percent of the lower 100 miles of the East Nishnabotna River. Continuous levees were constructed along each bank upstream of Hamburg from the material excavated during this channelization work (Iowa Natural Resources Council, 1955, p. 3,37 ). Channelization and levee construction has continued since 1929 for local sections of streams in the basin (U.S. Army Corps of Engineers, 1974, p. 6-7). Land use in the basin is predominantly agricultural with some livestock operations.

Normal annual precipitation (1951-1980) for the area is about 33 inches, based on data from the National Oceanic and Atmospheric Administration. Records of the USGS show that the mean annual runoff is from 5.4 to 7.0 inches for the Nishnabotna River basin.

\section{FLOOD HISTORY}

Floods in the Nishnabotna River basin have been documented since 1918 in annual reports of the USGS (see references). The first gaging station established in the river basin was on the East Nishnabotna River at Red Oak (06809500) in May 1918.

The active and discontinued USGS gaging stations used in this report are shown in figure 1, and are listed in table 1. Selected discharges and recurrence intervals for each of the major floods described in this section, and maximum, known flood-peak discharges for each gaging station are listed in table 1. Known flood peaks at miscellaneous, USGS, indirect-measurement sites are listed in table 2 , and the locations of these sites are shown in figure 2. Graphs of the annual peak discharges for six gaging stations are shown in figures 3-8. The graphs illustrate that the 1947,1958,1972,1982, and 1987 flood peaks are among the largest on record for these selected gaging stations.

Historical information concerning the magnitude and severity of floods that occurred in the Nishnabotna River basin before 1912 is scant. A report by the U.S. Army Corps of Engineers (1940, p. 19-26) lists damage-causing floods that reportedly occurred during $1849,1851,1867,1881,1882,1883$, 1902, and 1903, and descriptions of 12 floods which occurred from 1912 to 1939. The floods which occurred from 1912 to 1939 caused considerable damage, but are not reported as being record-high floods. The peak discharge on the East Nishnabotna River at Red Oak (06809500), on June 7, 1917, is the fourth largest on record (fig. 7).

The severe flooding that affected much of Iowa during June 1947, also involved the Nishnabotna River basin. Frequent rains from the latter half of May through June saturated the soils in the basin. The rain from four intense thunderstorms during June 1947 caused four distinct flood peaks in the Nishnabotna River basin (Iowa Natural Resources Council, 1955, p. 28-35). The first flood occurred in the East Nishnabotna River basin and was caused primarily by rainfall over the eastern tributaries of the East Nishnabotna River on June 1-2. Rainfall on June 3-5 in the lower part of the Nishnabotna River basin caused the second flood to occur primarily south of the Cass and Pottawattamie County lines on the West and East Nishnabotna Rivers and other tributaries. An intense thunderstorm on June 12-13 was so widespread, that it caused the third flood to be severe throughout the entire basin on the main streams and most tributaries along their entire lengths. The Iowa Natural Resources Council (1955) reports that two deaths were caused by this third flood in Red Oak. The combined effects of the antecedent moisture conditions and another storm on June 21-22 caused the fourth flood, which produced the maximum discharge for the period of record on the Nishnabotna River above Hamburg (06810000). A discharge of $55,500 \mathrm{ft}^{3} / \mathrm{s}$ (cubic feet per second) was 


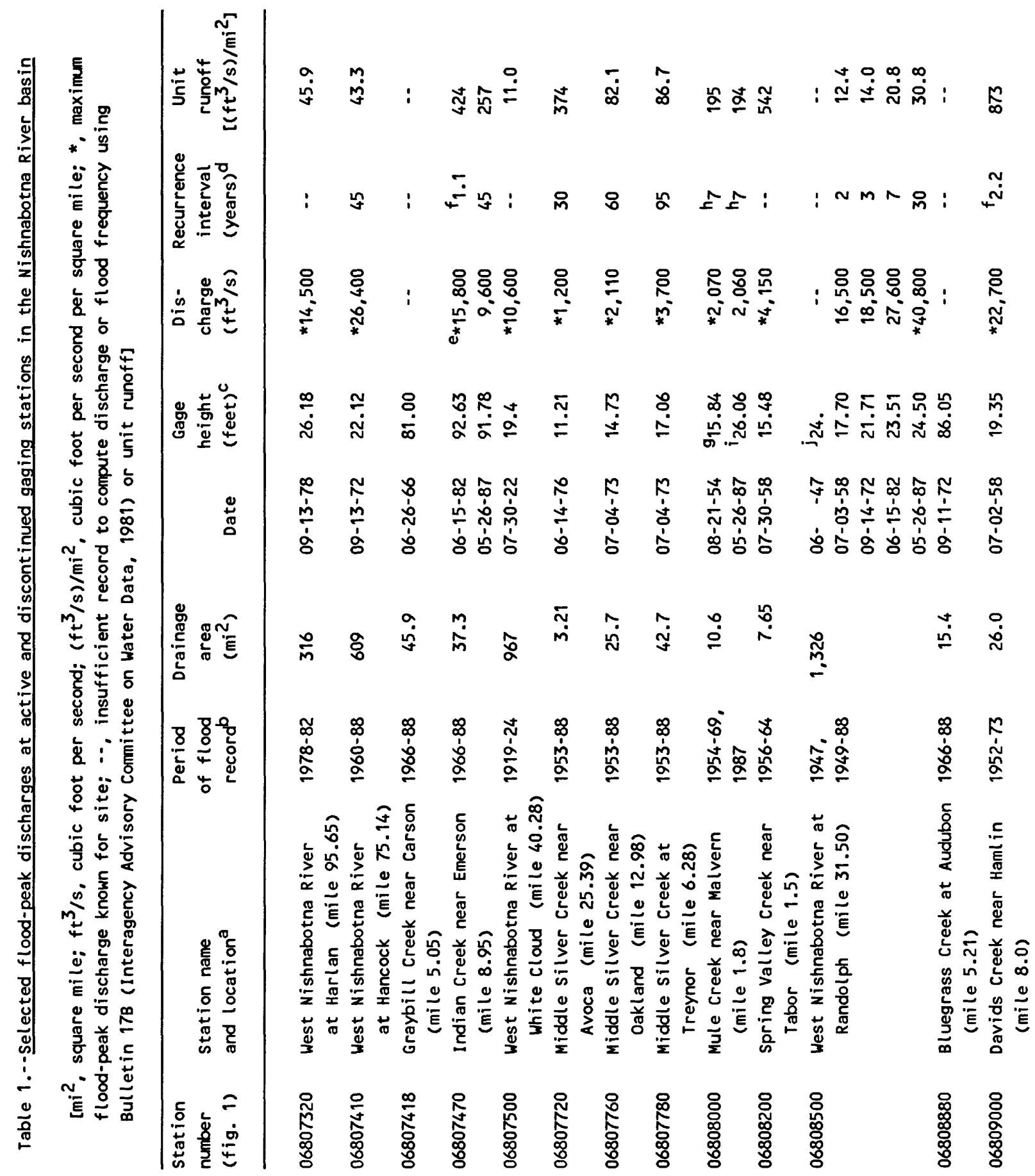




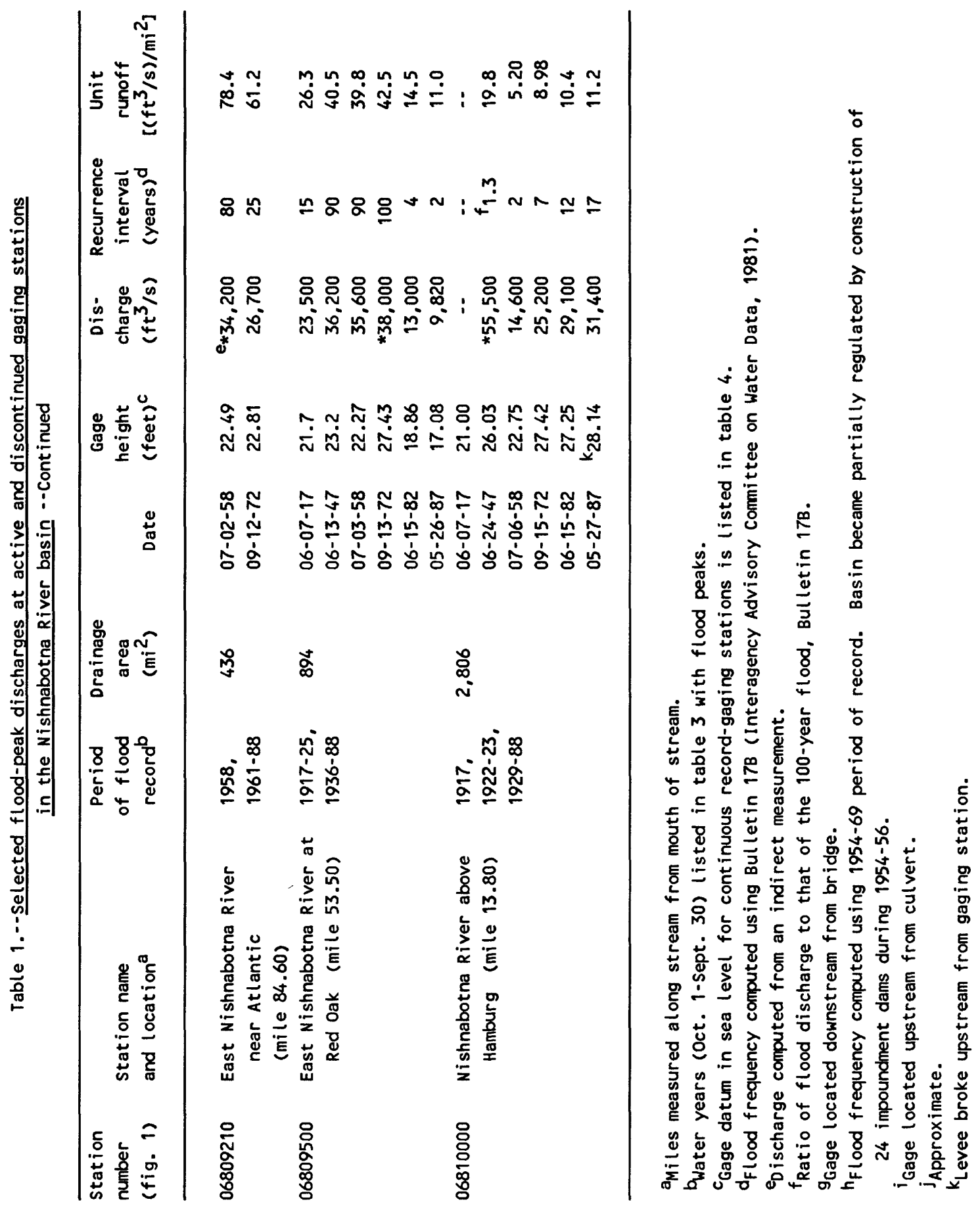




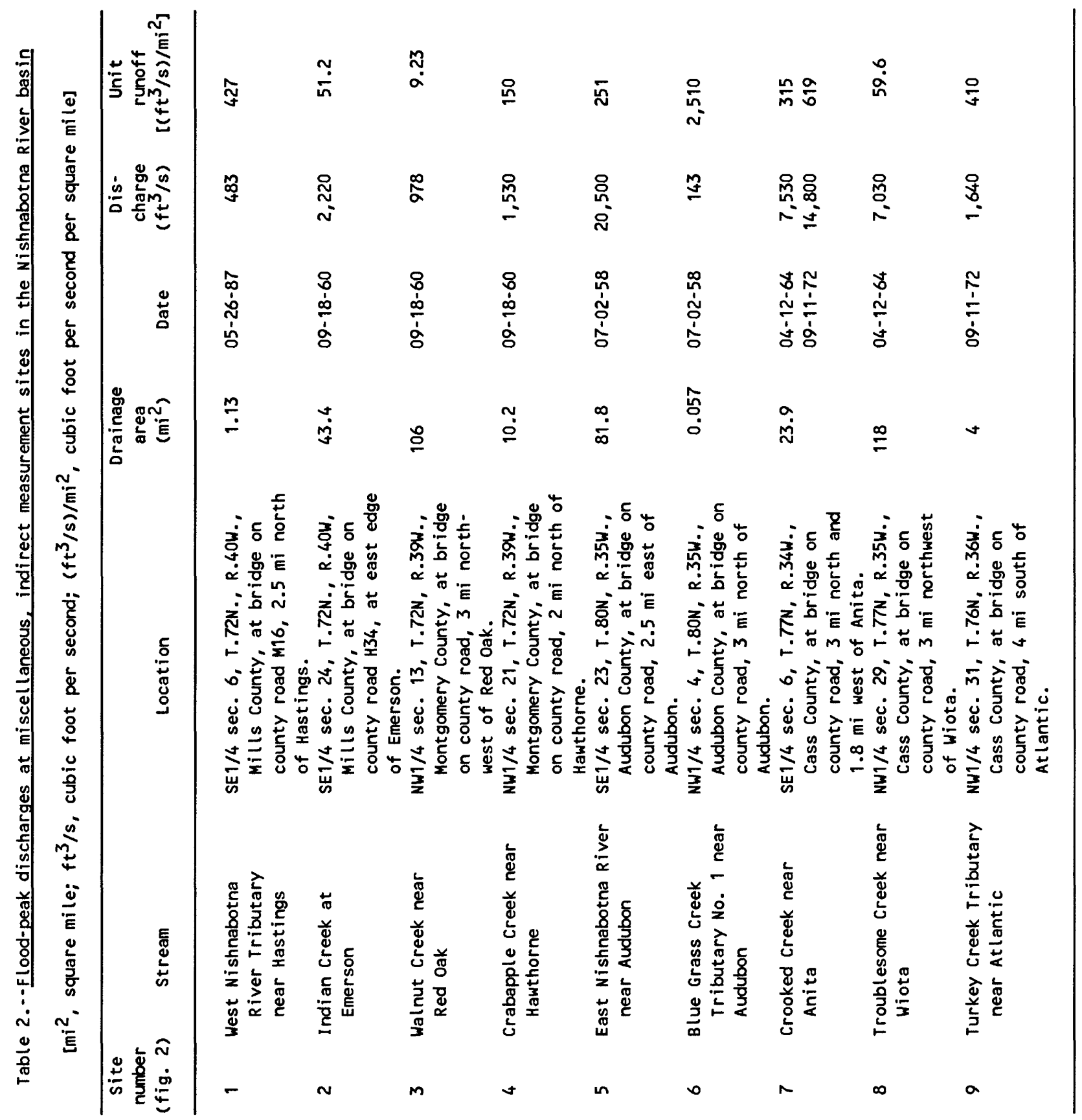








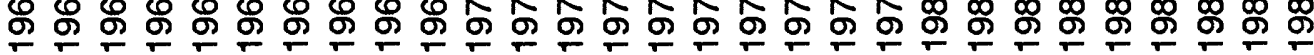

WATER YEAR

Figure 3.-- Annual peak discharges for period of record

for West Nishnabotna River at Hancock gaging station.



\section{WATER YEAR}

Figure 4.-- Annual peak discharges for period of record

for Indian Creek near Emerson crest-stage gaging station. 


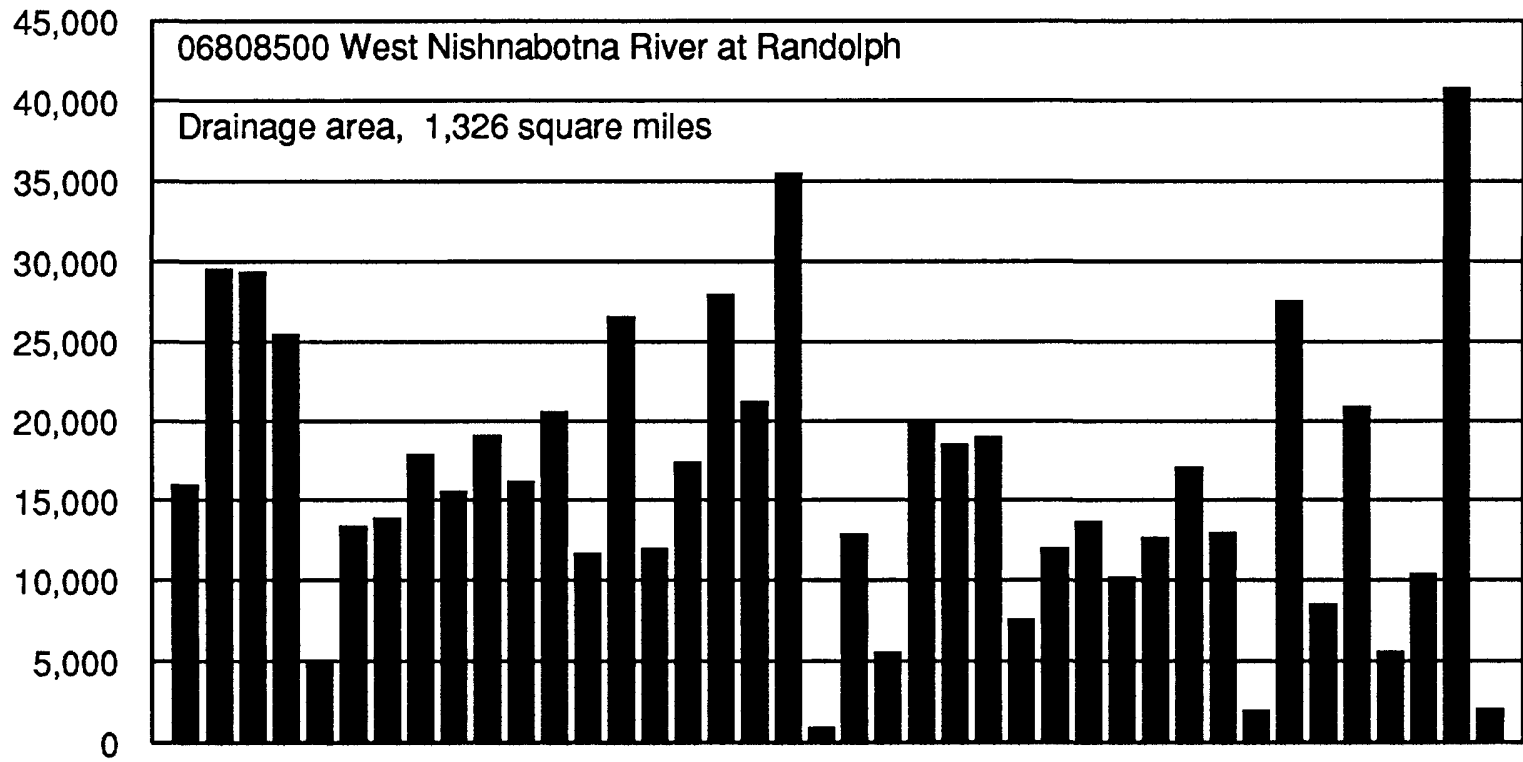

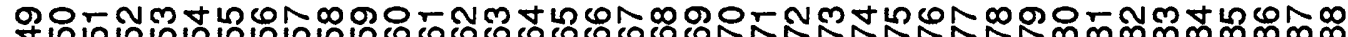



WATER YEAR

Figure 5.-- Annual peak discharges for period of record

for West Nishnabotna River at Randolph gaging station.

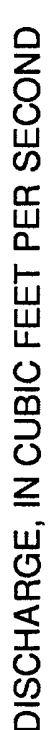

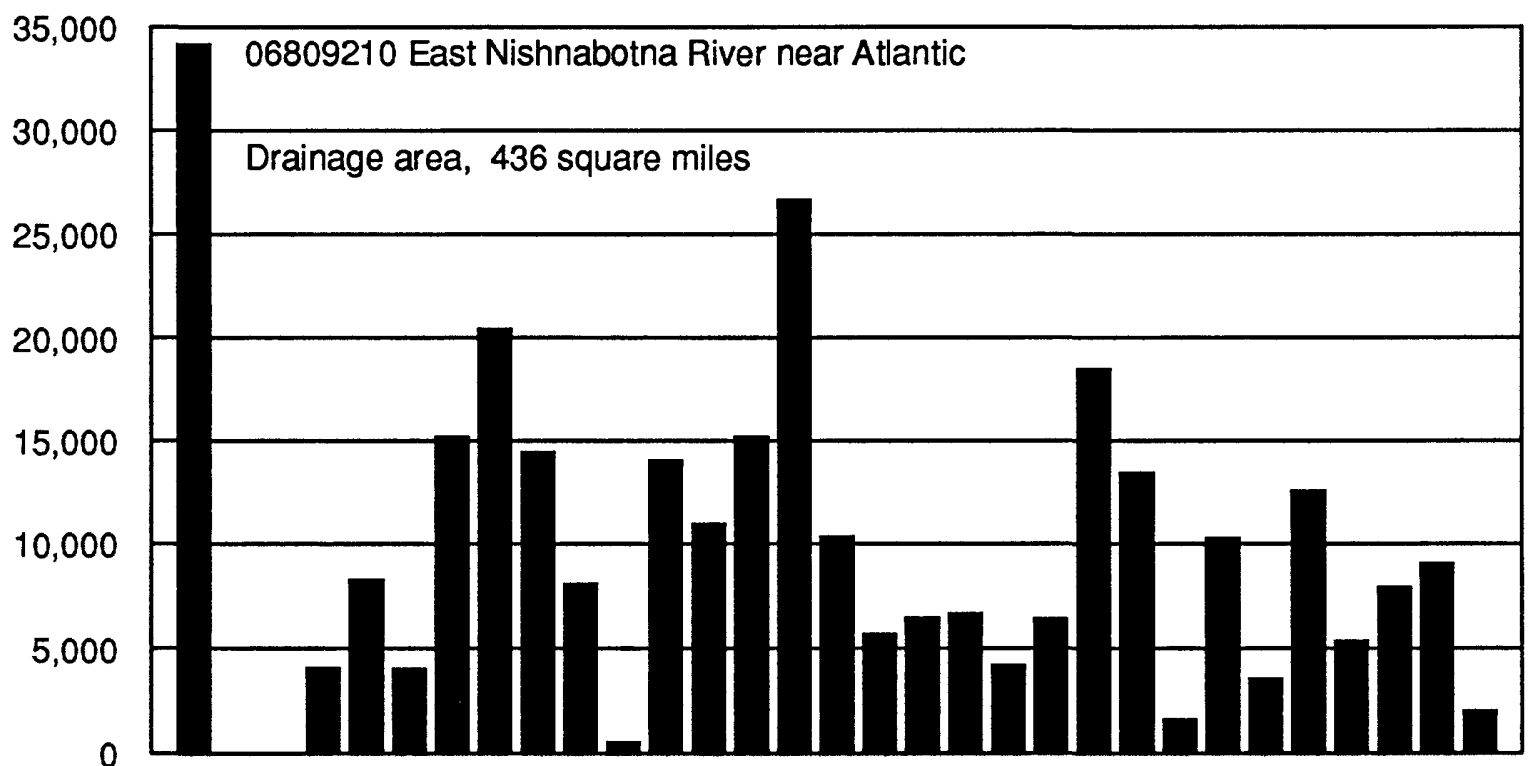

舟

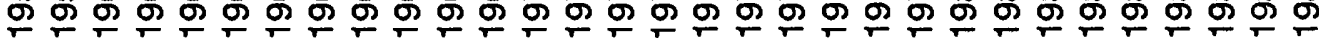

WATER YEAR

Figure 6.-- Annual peak discharges for period of record

for East Nishnabotna River near Atlantic gaging station. 


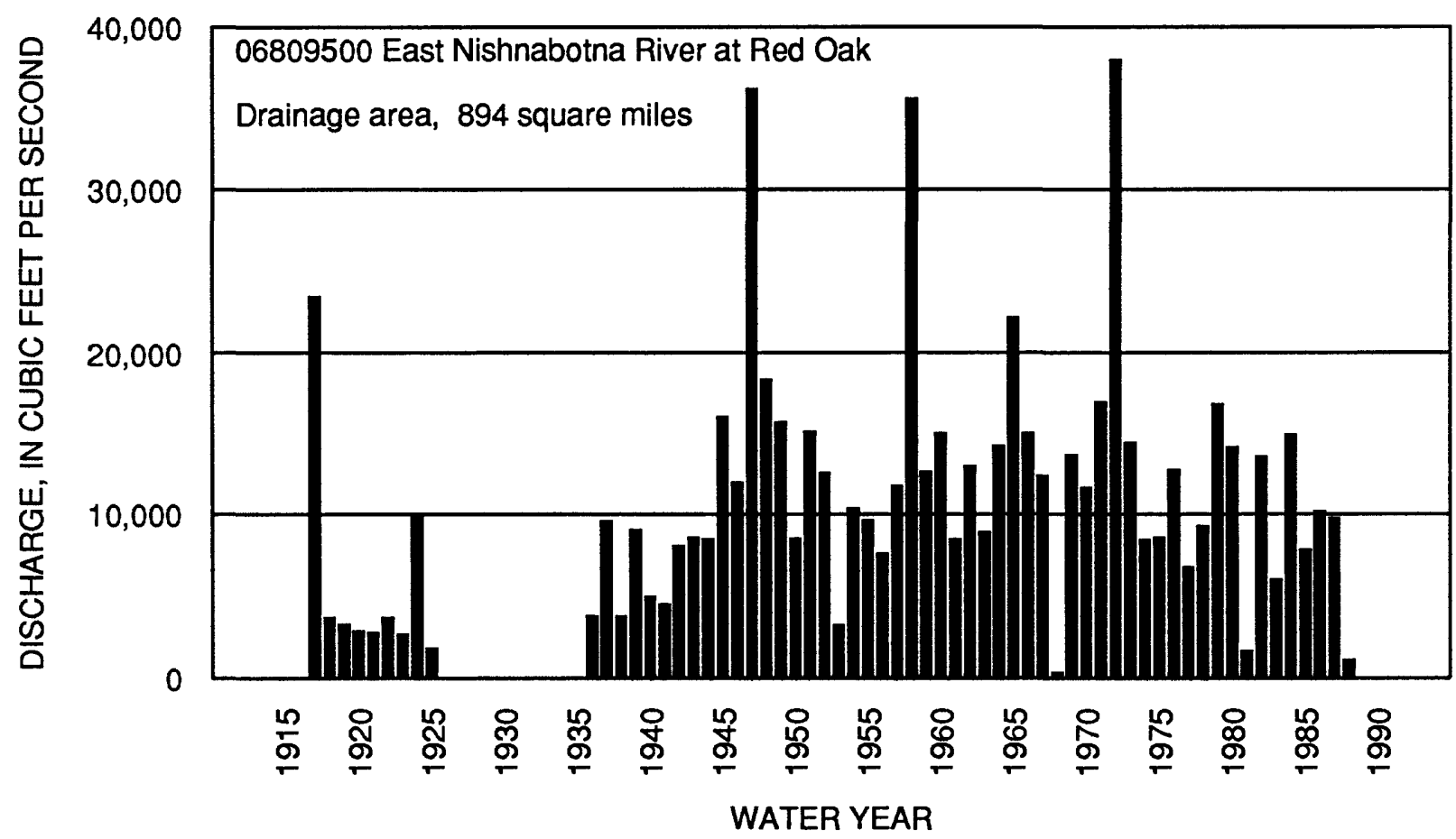

Figure 7.-- Annual peak discharges for period of record

for East Nishnabotna River at Red Oak gaging station.



Figure 8.-- Annual peak discharges for period of record

for Nishnabotna River above Hamburg gaging station. 
recorded for this site on June 24,1947 , which is 1.3 times larger than the Bulletin 17B, 100-year recurrence interval discharge (fig. 8). A discussion of usage and methodologies for determining the 100year recurrence interval follows in the "Flood Frequency and Magnitude" section. Damages in Fremont and Montgomery Counties from the June 1947 floods totaled \$2.4 million (U.S. Department of Commerce and Iowa Department of Agriculture, July 1947, p. 85).

The flood of July 1-3, 1958, in the East Nishnabotna River basin was caused by an intense thunderstorm centered above the headwaters of the basin during the night of July 1 . At the city of Audubon, 12.53 inches of rainfall were recorded during 24 hours on July 2; the second largest official amount of precipitation on record (1844-1981) for Iowa (Waite and Jaeger, 1981, p. 18-20, 40). This 24hour rainfall is almost twice the 100-year recurrence interval of 6.7 inches for the Audubon area. An unofficial rainfall of 13.23 inches also was reported by Waite and Jaeger for another Audubon site for the night of July 1 . The resulting flash flood was so sudden that 19 people were killed before protective measures could be taken (U.S. Army Corps of Engineers, 1959, p. 8-14). The Corps (1959) reported that total damages in the Nishnabotna River basin from the July 1-3, 1958, flood were $\$ 5.7$ million. At a gaging station near the area of greatest precipitation, Davids Creek near Hamlin (06809000), a peak discharge of $22,700 \mathrm{ft}^{3} / \mathrm{s}$ was recorded on July 2,1958 . This peak discharge is 2.2 times larger than the Bulletin 17B, 100-year recurrence interval discharge of $10,300 \mathrm{ft}^{3} / \mathrm{s}$, and it is one of the largest unit runoffs ever recorded in Iowa (873 cubic feet per second per square mile). At the gaging station, East Nishnabotna River near Atlantic (06809210), the peak discharge on July 2, 1958, is the maximum discharge for the period of record (fig. 6).

Severe flooding in the Nishnabotna River basin during September 10-15, 1972, resulted from an intense thunderstorm system over the upper part of the basin on September 10-12. At a site near Harlan, in Shelby County, 12.49 inches of rainfall was recorded on September 11, and the 3-day rainfall total was 20 inches (National Oceanic and Atmospheric Administration, September 1972, p. 136). The approximate 100-year, 3-day rainfall recurrence interval for Shelby County is 8.2 inches. Near Adair, in Adair County, 6.6 inches of rain fell in 4 hours during the evening of September 10 (U.S. Army Corps of Engineers 1974, p. 8-32). The Corps (1974) reported that total damages in the Nishnabotna River basin from the September $10-15,1972$, flood were $\$ 11.4$ million, and that two deaths were caused by washed-out bridges near Brayton. The flooding occurred in the latter part of the growing season, and crop losses were excessive. The Corps (1974) also reported crop damage estimates of $\$ 5.3$ million for the 108,000 acres affected by the flooding. Peak discharges from the September 1972 flood on the West Nishnabotna River at Hancock (06807410) and on the East Nishnabotna River at Red Oak (06809500) are the maximum discharges for the periods of record for these gaging stations (figs. 3 and 7).

During the night of June 14, 1982, an intense thunderstorm caused a devastating flood on Indian Creek in Mills and Montgomery Counties. Unofficial rainfalls of up to 8 inches in the headwaters of Indian Creek are reported by Heinitz (1985, p. 8), and unofficial rainfalls of up to 11 inches in the headwaters of both the West and East Nishnabotna Rivers are reported by the National Oceanic and Atmospheric Administration (June 1982, p. 26). The National Oceanic and Atmospheric Administration (June 1982) also reported that soil erosion from the storm exceeded 20 tons per acre in five southwestern Iowa counties, and that one death occurred during the night of June 14 when an Amtrak passenger train derailed at a wash out near Emerson. At the crest-stage gaging station, Indian Creek near Emerson (06807470), the peak discharge on June 15, 1982, is the maximum discharge for the period of record (fig. 4). This discharge of $15,800 \mathrm{ft}^{3} / \mathrm{s}$, is 1.1 times larger than the Bulletin $17 \mathrm{~B}$, 100-year recurrence interval discharge.

Intense rainfall on May 26, 1987, caused flooding to occur primarily in the lower West Nishnabotna River basin. The National Oceanic and Atmospheric Administration (May 1987, p. 27) reports that up to 10 inches of rain fell between 2 a.m. and nightfall on May 26 in parts of Mills and Montgomery Counties. Damages from the May 26-28, 1987, flood were \$5.5 million (Dennis Olson, Agriculture Stabilization and Conservation Service, oral commun., April 1988). The intense rainfall and flooding caused severe soil erosion, and damage to farm terraces and levees. Mills, Montgomery, 
Fremont, and Page Counties were declared Presidential and State disaster areas. The May 26, 1987, peak discharge on the West Nishnabotna River at Randolph (06808500) is the maximum discharge for the period of record (fig. 5).

\section{HYDROLOGIC DATA}

Gaging-station records are the primary source of data for analyzing and understanding the flood hydrology of a river basin. Flood information is obtained from complete-record gaging stations which provide a continuous chronology of streamflow, and from partial-record, crest-stage gaging stations which provide a chronology of annual peak flows. The locations of the active and discontinued USGS gaging stations used in this report are shown on the basin map in figure 1. Specific locations, annual peak stages and discharges, and other information pertaining to these gaging stations are given in table 3 (at end of report). Supplemental flood information is also obtained from miscellaneous measurement sites which provide streamflow data at sites where gaged data are not available. The locations of the miscellaneous measurement sites used in this report are shown in figure 2. Specific locations for these sites and flood-peak discharges, computed from indirect streamflow measurements, are given in table 2. Discharge records for these gaging stations and miscellaneous measurement sites are published in the annual streamflow reports of the USGS (see references).

The computation of discharge records at a gaging station is dependent upon the development of a relation between water surface elevations (stages) and the corresponding flow rates (discharges). The high-water portion of the stage-discharge relation, or rating curve as it is sometimes called, generally remains stable if the channel downstream from the gaging site remains unchanged. Changes in the stage-discharge relation occur from time to time, either gradually or abruptly, due to changes in the river channel that result from scour, deposition, or the growth of vegetation (Rantz and others, 1982, p. 328-360).

\section{FLOOD FREQUENCY AND MAGNITUDE}

A flood event of a magnitude which is expected to be equalled or exceeded once on the average during any 100-year period (recurrence interval) has been commonly used as a standard against which flood peaks are measured. This event, commonly termed the 100-year flood, has a 1 percent chance of being equalled or exceeded during any year. Although the recurrence interval represents the long-term average period between floods of a specific magnitude, rare floods could occur at short intervals or even within the same year.

The methodology for determining flood-flow frequency discharges is outlined by the United States Water Resources Council (Interagency Advisory Committee on Water Data, 1981, p. 1-28). The Water Resources Council recommends using the Pearson type III distribution with log transformation of the data as a base method for determining flood-flow frequency discharges. In this report, this methodology for determining flood-flow frequency discharges is referred to as "Bulletin 17B".

Another method for determining flood-flow frequency discharges at sites in Iowa, including those not gaged, is described by Lara (1987, p. 2-19). Lara used the physiographic characteristics of Iowa as a guide in defining the boundaries of five hydrologic regions. Regional equations were developed by using the annual flood-peak discharges for all gaged stations in a hydrological, homogeneous area, thereby reducing potential errors associated with nonrepresentative, short-term stations. For this reason, regional analysis also may produce improved estimates of the flood characteristics at gaged sites. Lara also used the Pearson type III distribution with log transformation as the base method for developing the regional equations.

The relation of maximum discharges and regional, 100-year flood discharges with drainage area for active and discontinued gaging stations, and for miscellaneous measurement sites in the Nishnabotna River basin are shown in figure 9. The curves shown in figure 9 represent the 100-year, 





flood-frequency equations developed by Lara for the three hydrologic regions delineated in figure 2 . The maximum discharges shown in figure 9 represent the five floods discussed in the "Flood History" section, in addition to other flood events listed in tables 1 and 2.

The Bulletin 17B and regional flood-peak discharges for selected recurrence intervals for the gaging stations in the Nishnabotna River basin are listed in table 4. Lara (1987, p. 13-15) used separately defined flood-peak discharges for the main stem stations on the West and East Nishnabotna Rivers (discharges are based on Bulletin 17B, flood-frequency computations through the 1984 water year).

The Nishnabotna River basin above the Hamburg gaging station (06810000) has parts of its drainage area in three of the hydrologic regions defined by Lara (1987); approximately 12 percent of the drainage area is in region 1, 78.5 percent is in region 2, and 9.5 percent is in region 3 (figs. 1 and 2). Therefore, regional flood-frequency estimates for intra-basin sites in the Nishnabotna River basin may necessitate the use of more than one regional, flood-frequency equation. For stations listed in table 4 with basins situated in more than one hydrologic region, weighted averages were used based on drainage-area ratios, to compute the regional flood-peak discharges.

The relation of Bulletin 17B and region 2, 100-year flood discharges with drainage area for the majority of streams in the Nishnabotna River basin are shown in figure 10. The estimated 100-year flood peaks for the main stems of the West and East Nishnabotna Rivers, defined separately by Lara, also are shown in figure 10. Data in table 4 can be used to plot comparisons of the regional 2-, 5-, 10-, 25- and 50-year flood discharges to those defined using Bulletin 17B, as was done for the 100-year discharges shown in figure 10.

Several Bulletin 17B estimates of 100-year discharges plot significantly above or below the region 2, regression-equation curve for 100-year discharges in figure 10. These variations between Bulletin 17B and region 2 estimates of 100-year discharges probably are because of flood attenuation, length of gaging station record, or differences in basin topography.

\section{FLOOD PROFILES}

Elevation profiles for the 1972 and 1987 floods on the West and East Nishnabotna Rivers are shown in figures 11-27 (follow references). The 1972 profiles are for 107 miles on the West Nishnabotna River, 84 miles on the East Nishnabotna River, and 4 miles on the Nishnabotna River. The 1987 profiles are for 47 miles on the lower West Nishnabotna River and 4 miles on the Nishnabotna River. A 9-mile profile of the 1982 flood on Indian Creek is shown in figure 28 (follow references). The profiles were defined by field data obtained by the U.S. Geological Survey. High-water marks located both upstream and downstream from bridges were identified within a few days of passage of the flood peaks and were referenced to a common datum by leveling. Profiles between the bridges are straight-line interpolations, which only provide an approximation of water-surface elevations which occurred between the bridges.

A 1989 low-water profile also is shown in figures 11-27 to indicate the approximate range of stage that can occur within the profiled reaches. The 1989 low-water profile closely parallels a lowwater profile made during a slightly larger discharge period on November 1-2, 1978. The 1978 lowwater profile, which is not shown in figures 11-27, plots slightly above the 1989 low-water profile throughout most of the profiled reaches.

The June 15, 1982, flood profile for Indian Creek, shown in figure 28, extends from the U.S. Highway 34 bridge downstream to near the mouth. As shown on the profile, floodwaters within the town of Emerson were virtually pooled for about one-half mile along the upstream side of the railroad grade. A low-water profile taken on September 14, 1982, closely approximates the channel bottom configuration and can be used to estimate the depths of the water for the June 15, 1982, flood. 
Table 4.-- $D$ ischarge and frequency of flood flows for active and discontinued gaging stations in the Nishnabotna River basin

[17B, Bulletin 17B (Interagency Advisory Committee on Water Data, 1981); --, insufficient record to compute flood frequency using Bulletin 17B; Lara, flood-frequency equations, regions 1-3 (Lara, 1987, p. 28). For stations with basins situated in more than one hydrologic region, weighted averages were used based on drainage-area ratios. Separately defined flood-frequency discharges were used for the main stem stations on the West and East Nishnabotna Rivers.]

\begin{tabular}{|c|c|c|c|c|c|c|c|c|}
\hline \multirow{2}{*}{$\begin{array}{l}\text { Station } \\
\text { number }\end{array}$} & \multirow[b]{2}{*}{ Station name } & \multirow[b]{2}{*}{ Method } & \multicolumn{6}{|c|}{$\begin{array}{l}\text { Discharge, in cubic feet per second, } \\
\text { for indicated recurrence interval, in years }\end{array}$} \\
\hline & & & 2 & 5 & 10 & 25 & 50 & 100 \\
\hline 06807320 & $\begin{array}{c}\text { W. Nishnabotna R. } \\
\text { at Harlan }\end{array}$ & $\begin{array}{l}178 \\
\text { Lara }\end{array}$ & 4,860 & 8,640 & $\begin{array}{c}\cdots \\
11,700\end{array}$ & 15,500 & 18,300 & 21,600 \\
\hline 06807410 & $\begin{array}{l}\text { W. Ni Shnabotna R. } \\
\text { at Hancock }\end{array}$ & $\begin{array}{l}17 \mathrm{~B} \\
\text { Lara }\end{array}$ & $\begin{array}{l}8,950 \\
9,460\end{array}$ & $\begin{array}{l}14,800 \\
15,500\end{array}$ & $\begin{array}{l}18,600 \\
19,500\end{array}$ & $\begin{array}{l}23,400 \\
24,300\end{array}$ & $\begin{array}{l}26,800 \\
27,700\end{array}$ & $\begin{array}{l}30,000 \\
30,900\end{array}$ \\
\hline 06807418 & $\begin{array}{c}\text { Graybill Creek } \\
\text { near Carson }\end{array}$ & $\begin{array}{l}178 \\
\text { Lara }\end{array}$ & 1,740 & 3,300 & 4,680 & 6,480 & 7,750 & 9,350 \\
\hline 06807470 & $\begin{array}{l}\text { Indian Creek } \\
\text { near Emerson }\end{array}$ & $\begin{array}{l}\text { a } 17 \mathrm{~B} \\
\text { Lara }\end{array}$ & $\begin{array}{r}783 \\
1,540\end{array}$ & $\begin{array}{l}2,200 \\
2,940\end{array}$ & $\begin{array}{l}3,790 \\
4,170\end{array}$ & $\begin{array}{l}6,790 \\
5,800\end{array}$ & $\begin{array}{l}9,940 \\
6,940\end{array}$ & $\begin{array}{r}14,000 \\
8,370\end{array}$ \\
\hline 06807500 & $\begin{array}{l}\text { W. Nishnabotna R. } \\
\text { at White Cloud }\end{array}$ & $\begin{array}{l}17 \mathrm{~B} \\
\text { Lara }\end{array}$ & $9, \overline{410}$ & 16,600 & 22,900 & 30,500 & 35,400 & 42,300 \\
\hline 06807720 & $\begin{array}{c}\text { Middle Silver } \mathrm{Cr} \text {. } \\
\text { near Avoca }\end{array}$ & $\begin{array}{l}b_{178} \\
\text { Lara }\end{array}$ & $\begin{array}{l}387 \\
381\end{array}$ & $\begin{array}{l}679 \\
763\end{array}$ & $\begin{array}{r}879 \\
1,080\end{array}$ & $\begin{array}{l}1,130 \\
1,540\end{array}$ & $\begin{array}{l}1,310 \\
1,890\end{array}$ & $\begin{array}{l}1,480 \\
2,280\end{array}$ \\
\hline 06807760 & $\begin{array}{c}\text { Middle Silver } \mathrm{Cr} \text {. } \\
\text { near Oakland }\end{array}$ & $\begin{array}{l}17 B \\
\text { Lara }\end{array}$ & $\begin{array}{r}870 \\
1,250\end{array}$ & $\begin{array}{l}1,260 \\
2,400\end{array}$ & $\begin{array}{l}1,520 \\
3,400\end{array}$ & $\begin{array}{l}1,850 \\
4,740\end{array}$ & $\begin{array}{l}2,090 \\
5,700\end{array}$ & $\begin{array}{l}2,320 \\
6,870\end{array}$ \\
\hline 06807780 & $\begin{array}{c}\text { Middle Silver } \mathrm{Cr} \text {. } \\
\text { at Treynor }\end{array}$ & $\begin{array}{l}\text { b }_{17 B} \\
\text { Lara }\end{array}$ & $\begin{array}{l}1,340 \\
1,780\end{array}$ & $\begin{array}{l}1,960 \\
3,550\end{array}$ & $\begin{array}{l}2,380 \\
5,140\end{array}$ & $\begin{array}{l}2,930 \\
7,050\end{array}$ & $\begin{array}{l}3,340 \\
9,070\end{array}$ & $\begin{array}{r}3,760 \\
11,100\end{array}$ \\
\hline 06808000 & $\begin{array}{l}\text { Mule Creek } \\
\text { near Malvern }\end{array}$ & $\begin{array}{l}c_{17 B} \\
\text { Lara }\end{array}$ & $\begin{array}{l}762 \\
912\end{array}$ & $\begin{array}{l}1,840 \\
2,070\end{array}$ & $\begin{array}{l}2,730 \\
3,120\end{array}$ & $\begin{array}{l}3,980 \\
4,380\end{array}$ & $\begin{array}{l}4,950 \\
6,180\end{array}$ & $\begin{array}{l}5,930 \\
7,750\end{array}$ \\
\hline 06808200 & $\begin{array}{l}\text { Spring Valley } \mathrm{Cr} \text {. } \\
\text { near Tabor }\end{array}$ & $\begin{array}{l}17 \mathrm{~B} \\
\text { Lara }\end{array}$ & $\begin{array}{l}\cdots \\
745\end{array}$ & 1,700 & 2,570 & 3,640 & 5,080 & 6,370 \\
\hline 06808500 & $\begin{array}{c}\text { W. Nishnabotna R. } \\
\text { at Randolph }\end{array}$ & $\begin{array}{l}\text { 17B } \\
\text { Lara }\end{array}$ & $\begin{array}{l}14,900 \\
15,900\end{array}$ & $\begin{array}{l}25,000 \\
23,100\end{array}$ & $\begin{array}{l}31,500 \\
27,600\end{array}$ & $\begin{array}{l}39,000 \\
32,700\end{array}$ & $\begin{array}{l}44,100 \\
36,200\end{array}$ & $\begin{array}{l}48,800 \\
39,400\end{array}$ \\
\hline
\end{tabular}


Table 4.--Discharge and frequency of flood flows for active and discontinued gaging stations gaging stations in the Nishnabotna River basin --Continued

\begin{tabular}{|c|c|c|c|c|c|c|c|c|}
\hline \multirow{2}{*}{$\begin{array}{l}\text { Station } \\
\text { number }\end{array}$} & \multirow[b]{2}{*}{ Station name } & \multirow[b]{2}{*}{ Method } & \multicolumn{6}{|c|}{$\begin{array}{l}\text { Discharge, in cubic feet per second, } \\
\text { for indicated recurrence interval, in years }\end{array}$} \\
\hline & & & 2 & 5 & 10 & 25 & 50 & 100 \\
\hline \multirow[t]{2}{*}{06808880} & Bluegrass Creek & $17 \mathrm{~B}$ & - & -- & -. & -- & -- & -- \\
\hline & at Audubon & Lara & 891 & 1,720 & 2,430 & 3,400 & 4,100 & 4,950 \\
\hline \multirow[t]{2}{*}{06809000} & Davids Creek & $17 \mathrm{~B}$ & 892 & 2,140 & 3,400 & 5,590 & 7,710 & 10,300 \\
\hline & near Haml in & Lara & 1,040 & 1,960 & 2,680 & 3,680 & 4,440 & 5,300 \\
\hline \multirow[t]{2}{*}{06809210} & E. Nishnabotna R. & $17 B$ & 8,600 & 15,400 & 20,300 & 26,800 & 31,800 & 36,700 \\
\hline & near Atlantic & Lara & 8,830 & 15,200 & 19,500 & 25,000 & 28,900 & 32,800 \\
\hline \multirow[t]{2}{*}{06809500} & E. Nishnabotna R. & $17 \mathrm{~B}$ & 9,400 & 16,700 & 21,700 & 28,000 & 32,600 & 37,100 \\
\hline & at Red Oak & Lara & 9,590 & 16,800 & 21,900 & 28,500 & 33,400 & 38,200 \\
\hline \multirow[t]{2}{*}{06810000} & Nishnabotna River & $17 \mathrm{~B}$ & 15,500 & 23,600 & 28,600 & 34,300 & 38,200 & 41,800 \\
\hline & above Hamburg & Lara & 15,600 & 23,500 & 28,200 & 33,700 & 37,400 & 40,800 \\
\hline
\end{tabular}

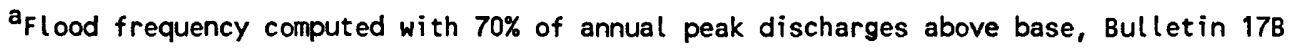
recommends the use of $75 \%$ of annual peak discharges above base.

$b_{F l o o d}$ frequency computed using annual peak discharges through the 1986 water year.

$c_{F l o o d}$ frequency computed using 1954-69 period of record. Basin became partially regulated by construction of 24 impoundment dams during 1954-56. 


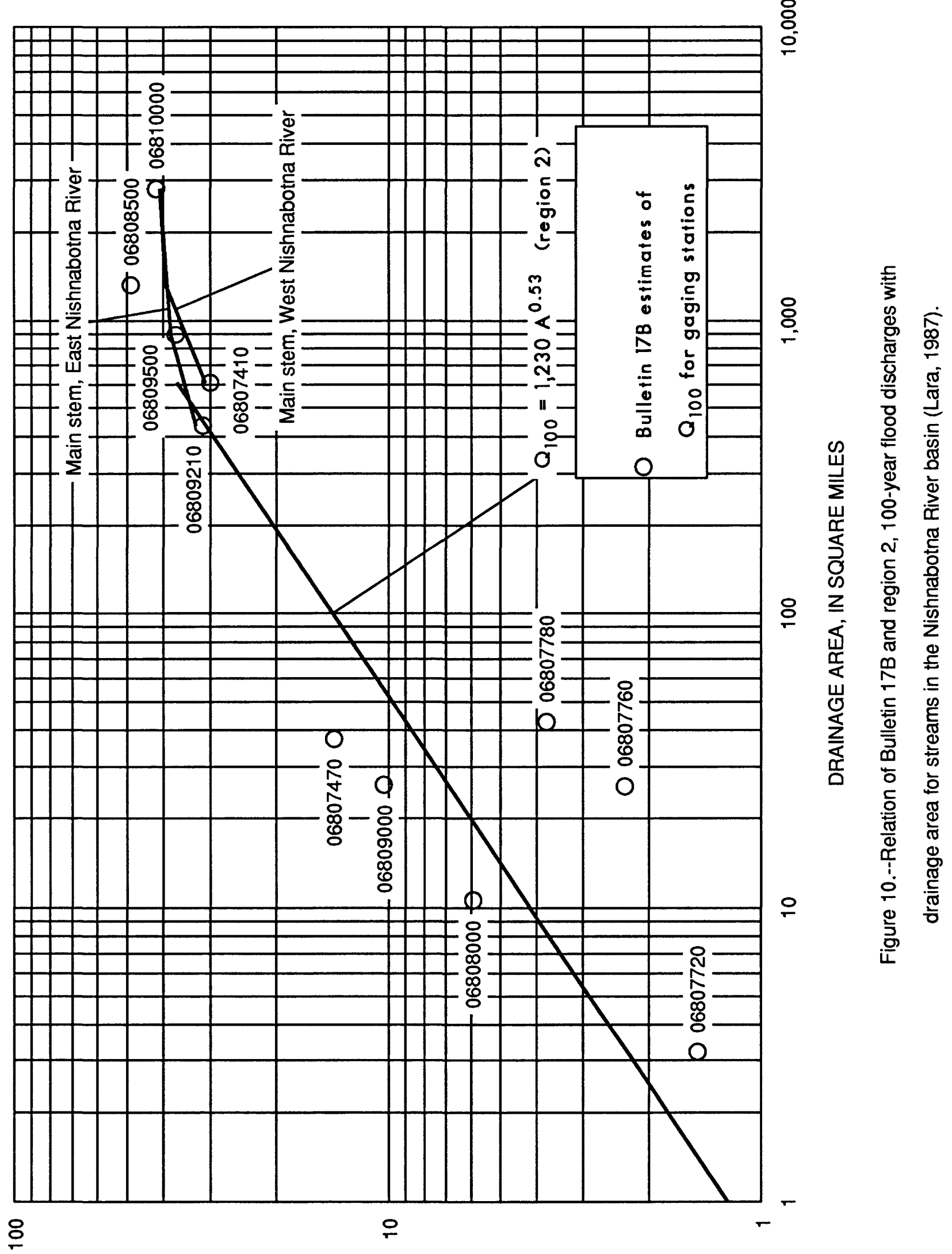


Bridge deck and low-bridge chord elevations are shown in figures 11-28 to indicate the relation between the elevation of the bridges and the elevations of the profiled floods and the low-water profiles. For sloping bridges, the profiled bridge deck and low-bridge chord elevations represent the lower ends of the bridges. Selected gaging-station discharges and recurrence intervals for the floods profiled in figures $11-28$ are listed in table 1.

In order to reference all the points along the profiles to a common datum, extensive leveling work was performed. At least one bench mark and one reference point were established at each bridge in the profiled reaches. Bench mark and reference point descriptions and elevations are listed in table 5 (at end of report).

River mileages, determined from the best available Geological Survey maps, are referenced to the mouth of the Nishnabotna River. Bridges, and a few other points, are designated by an index number that helps to identify their location. For example, 7241-24NW refers to a location in township 72 north, range 41 west, northwest $1 / 4$ section 24 .

\section{DISCUSSION}

The user of this report is cautioned that the stage-discharge data presented herein are representative of the physical conditions of the basin at the time of the flood events shown. Changes in the basin can alter the flood magnitude for a given frequency. Examples of these changes include, but are not limited to, extensive urbanization, implementation of agricultural conservation practices, installation of drainage systems, and construction of reservoirs. Changes in the channel conditions immediately downstream from a site can materially affect the stage-discharge relation. Examples of such changes include the construction of dams, bridges, or levees; changes in the flood-plain vegetative cover; straightening of the channel; and natural scour and fill. Temporary changes can be caused by ice and debris jams which produce backwater conditions and may cause the flood elevations to plot higher than the normal profile. 


\section{REFERENCES}

Heinitz, A.J., 1985, Floods of June - July, 1982, in Iowa: U.S. Geological Survey Open-File Report 85$151,18 \mathrm{p}$.

Interagency Advisory Committee on Water Data, 1981, Guidelines for determining flood flow frequency (2d ed., revised) [editorial corrections made March 1982]: Reston, Va., U.S. Geological Survey Office of Water Data Coordination, Hydrology Subcommittee Bulletin 17B, appendices 1-14, 28 p.

Iowa Natural Resources Council, 1955, An inventory of water resources and water problems, Nishnabotna River basin, Iowa: Des Moines, Iowa, Iowa Natural Resources Council Bulletin no. $2,61 \mathrm{p}$.

Lara, O.G., 1987, Method for estimating the magnitude and frequency of floods at ungaged sites on unregulated rural streams in Iowa: Iowa City, Iowa, U.S. Geological Survey Water-Resources Investigation Report 87-4132, $34 \mathrm{p}$.

Novak, C.E., 1985 edition, WRD data reports: preparation guide: U.S. Geological Survey, Water Resources Division, 325 p.

Rantz, S.E. and others, 1982, Measurement and computation of streamflow: volume 2. Computation of discharge: Washington, U.S. Geological Survey Water-Supply Paper 2175, p. 285-631.

U.S. Army Corps of Engineers, 1940, Survey report on flood control, Nishnabotna River basin, Iowa and Missouri: Examinations of Rivers and Harbors, 78th Congress, 1st Session, Jan. 6-Dec. 21, 1943: Washington, U.S. Government Printing Office, House Document No. 253, 35 p.

1959, Report on the flood of July 1958 in the Nishnabotna River basin, Iowa: Omaha, Nebr., 30 p.

1974, Nishnabotna River basin, Iowa: Report on flood of September 1972: Omaha, Nebr., 79 p.

National Oceanic and Atmospheric Administration, 1982, 1982, and 1987, Climatological data, Iowa: Asheville, N.C., National Climatic Center, monthly summaries, v. 83, no. 9., p. 133-150; v. 93, no. $6 ., 30$ p.; v. 98 , no. $5 ., 30$ p.

U.S. Department of Commerce, Weather Bureau, and Iowa Department of Agriculture, 1947, Climatological data, Iowa: Des Moines, monthly summary, v. 58, no. 7, p. 73--88.

U.S. Geological Survey, issued annually to 1960, Surface-water supply of the United States, part 6-B, Missouri River basin below Sioux City, Iowa, U.S. Geological Survey Water-Supply Papers.

U.S. Geological Survey,issued annually since 1961, Water Resources data for Iowa, U.S. Geological Survey Open-File Reports, Iowa City, Iowa, U.S. Geological Survey.

Waite, P., and Jaeger, P., 1981, Iowa's greatest 24-hour precipitation and related rain storm data: Des Moines, Iowa, Iowa Department of Agriculture, State Climatology Office, Climatology of Iowa Series 3, $45 \mathrm{p}$. 


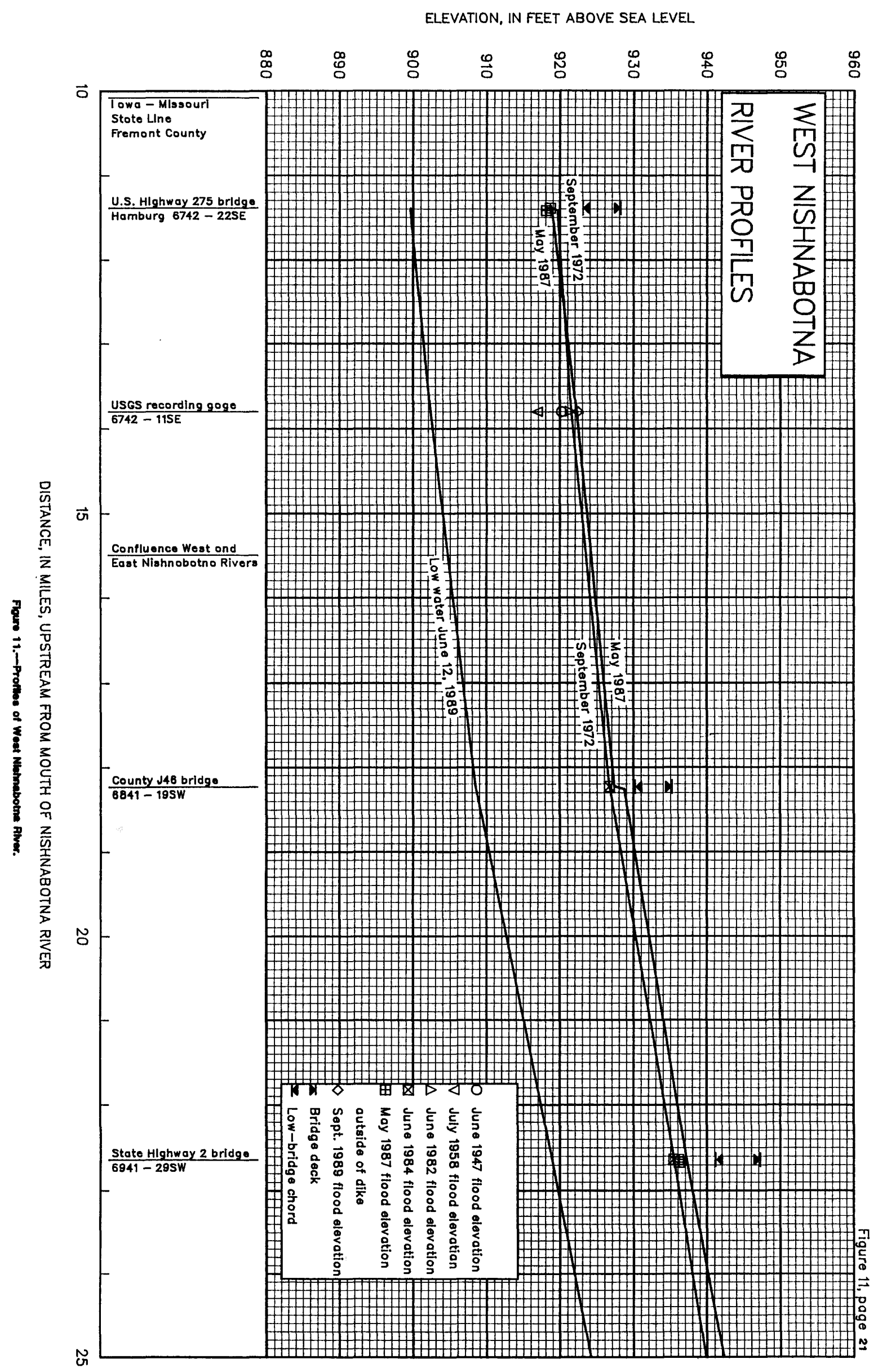


ELEVATION, IN FEET ABOVE SEA LEVEL

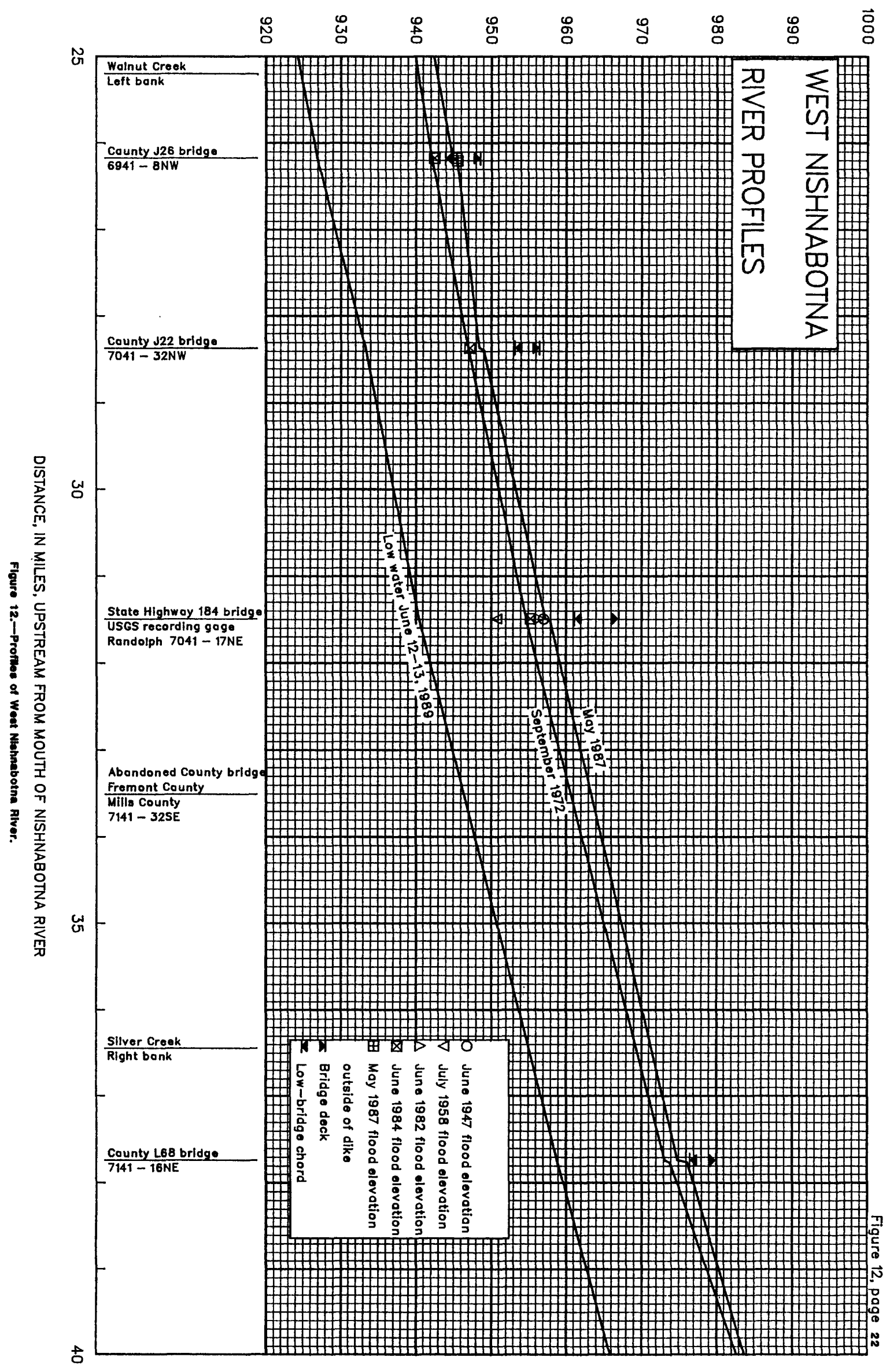


ELEVATION, IN FEET ABOVE SEA LEVEL

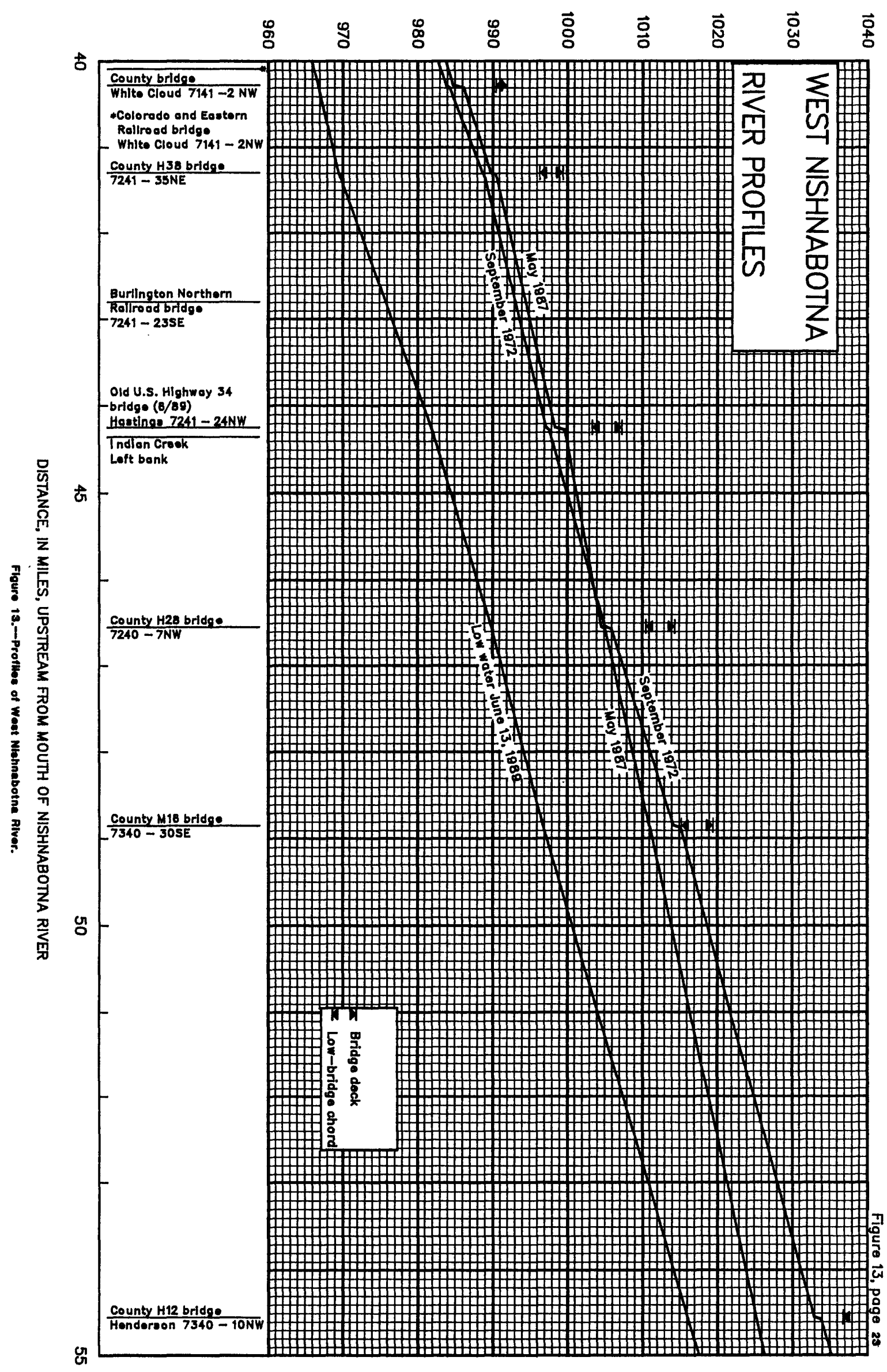




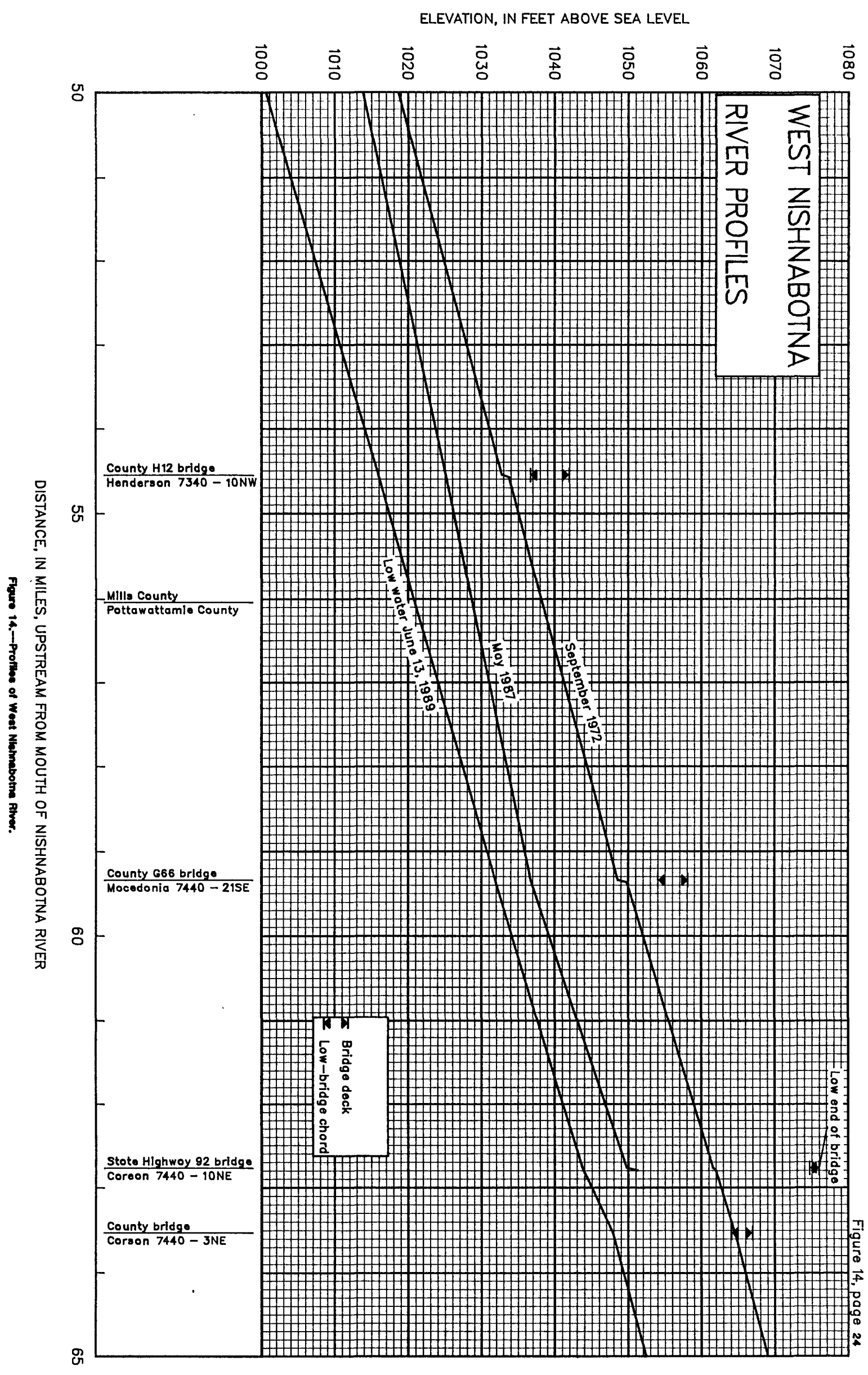




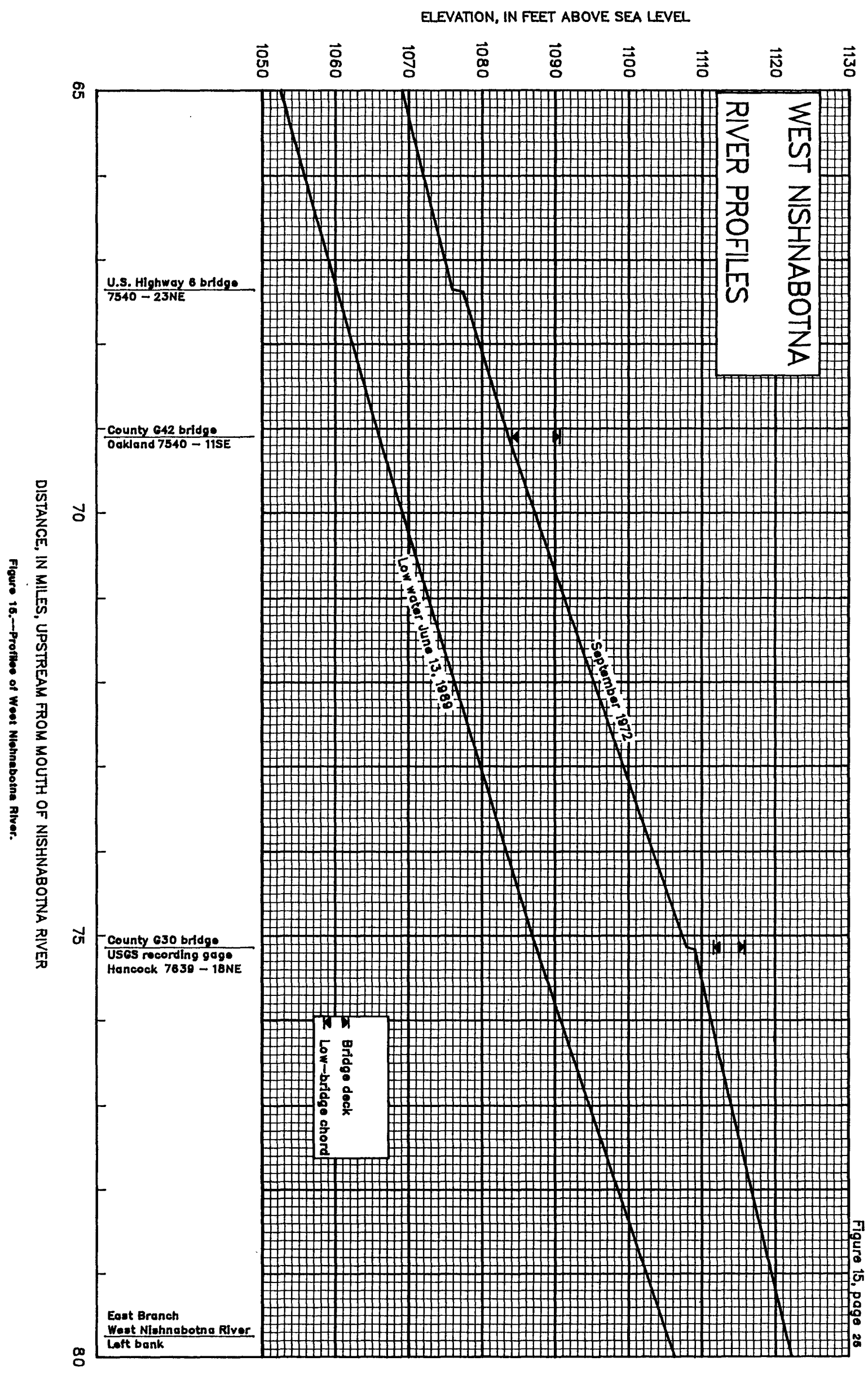




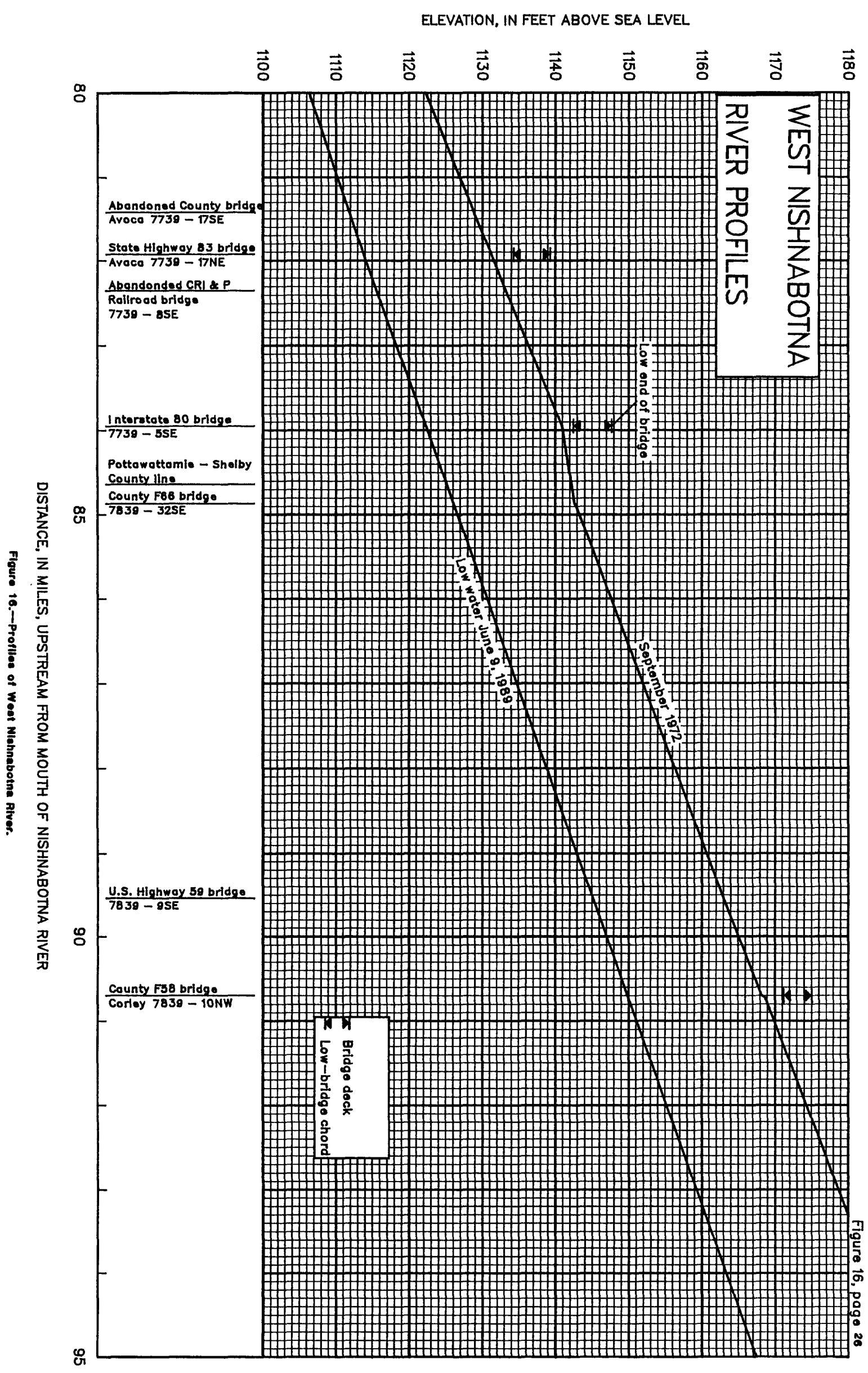




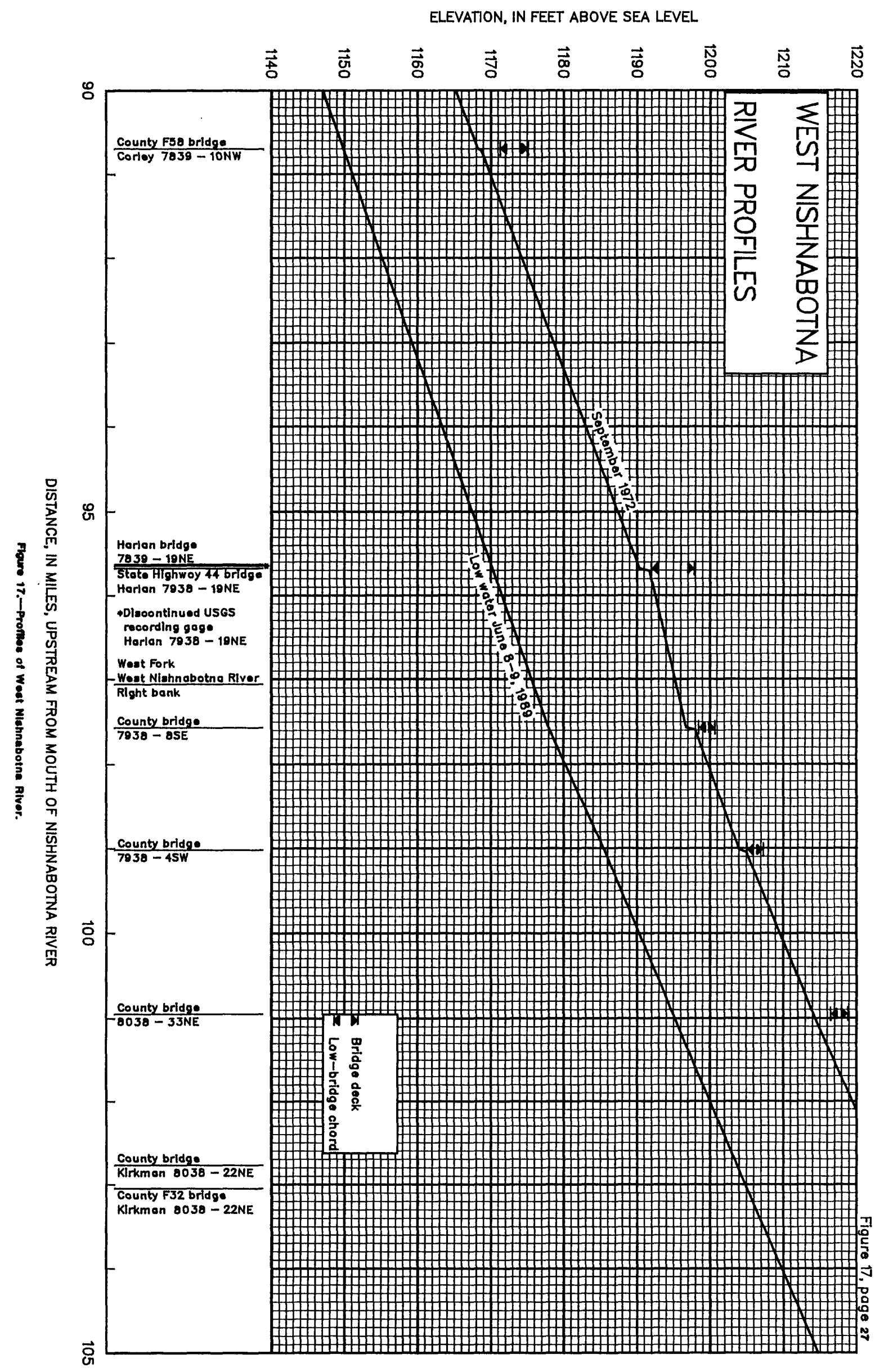


ELEVATION, IN FEET ABOVE SEA LEVEL

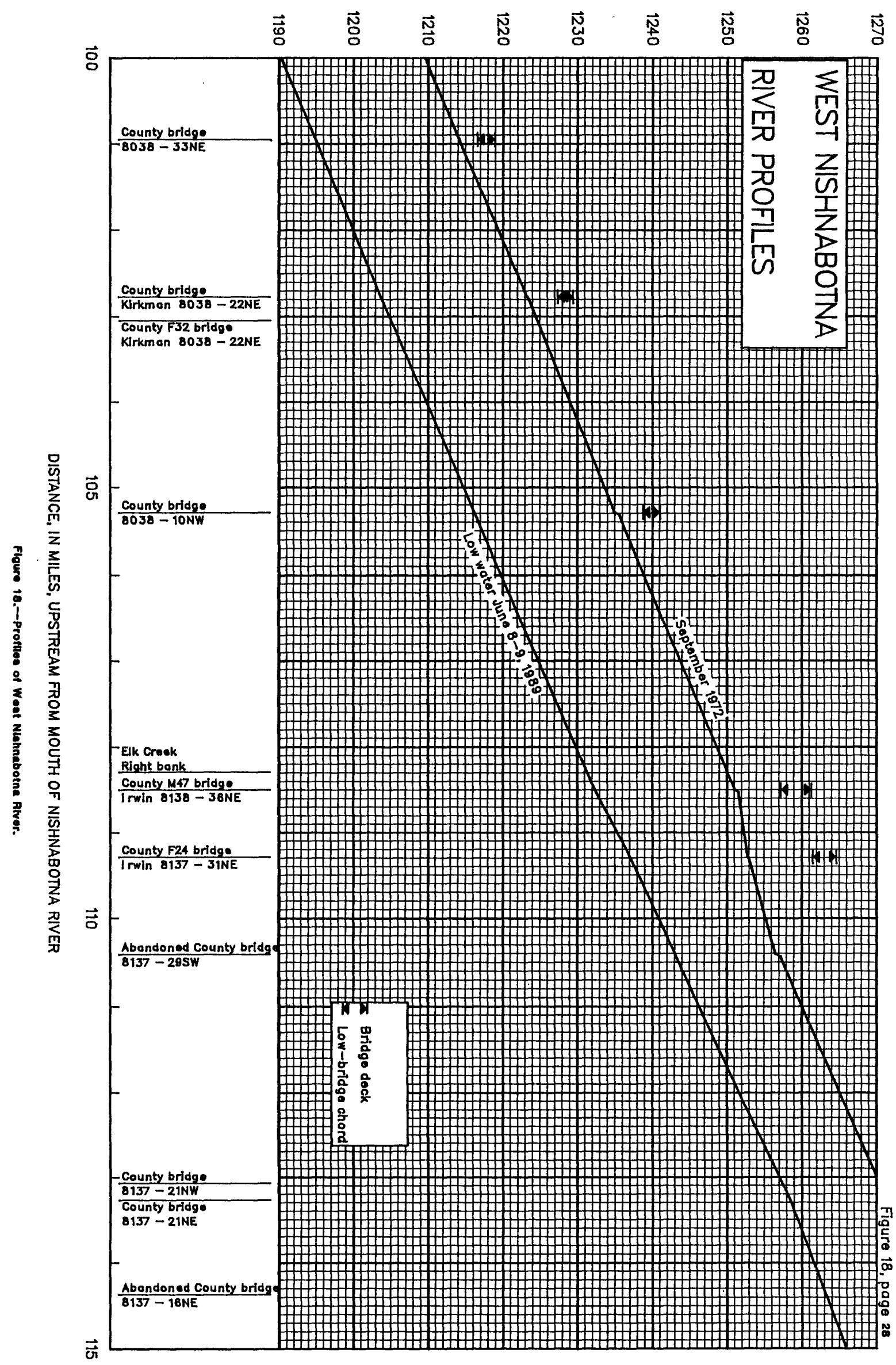


ELEVATION, IN FEET ABOVE SEA LEVEL

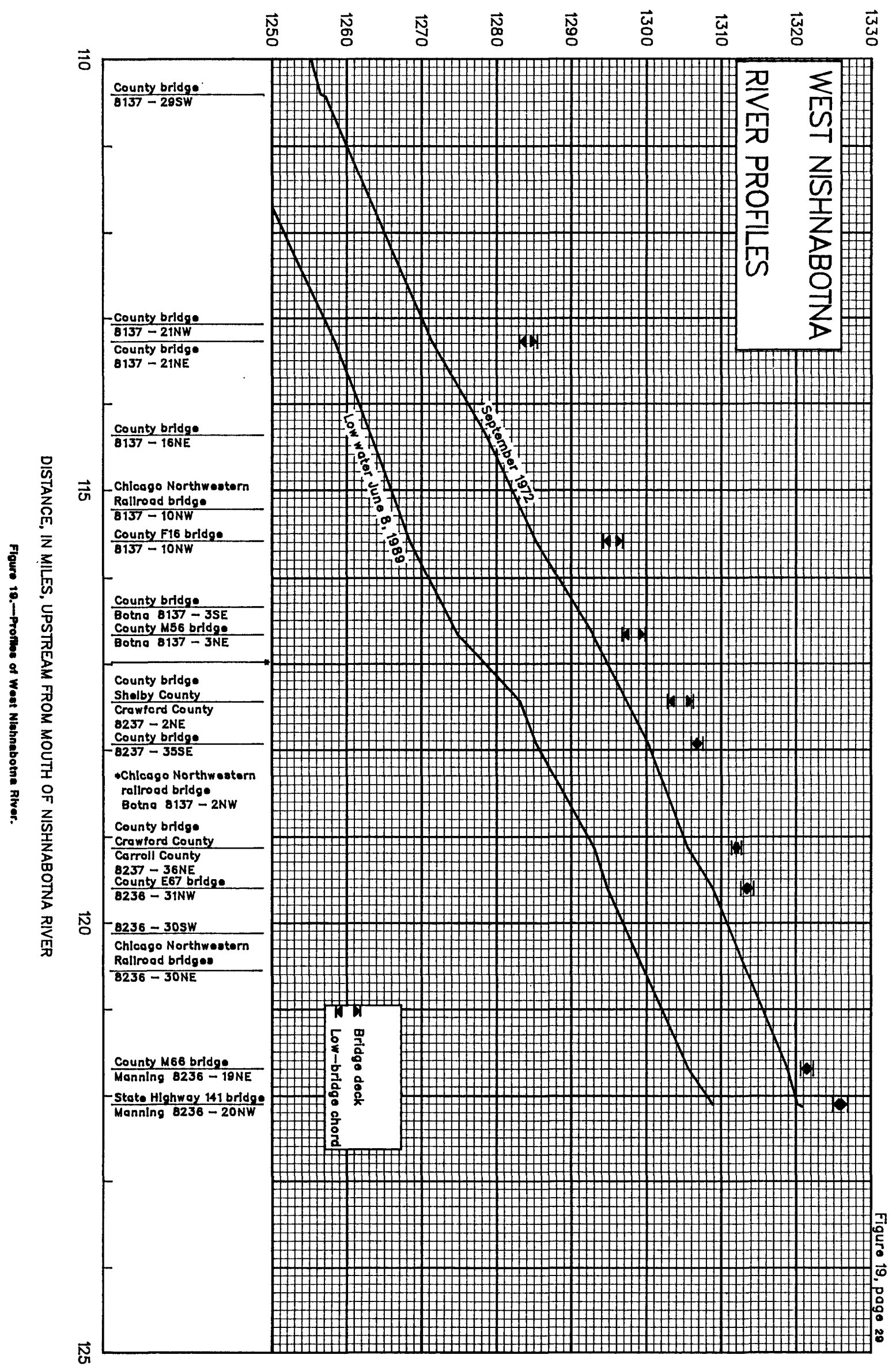


ELEVATION, IN FEET ABOVE SEA LEVEL

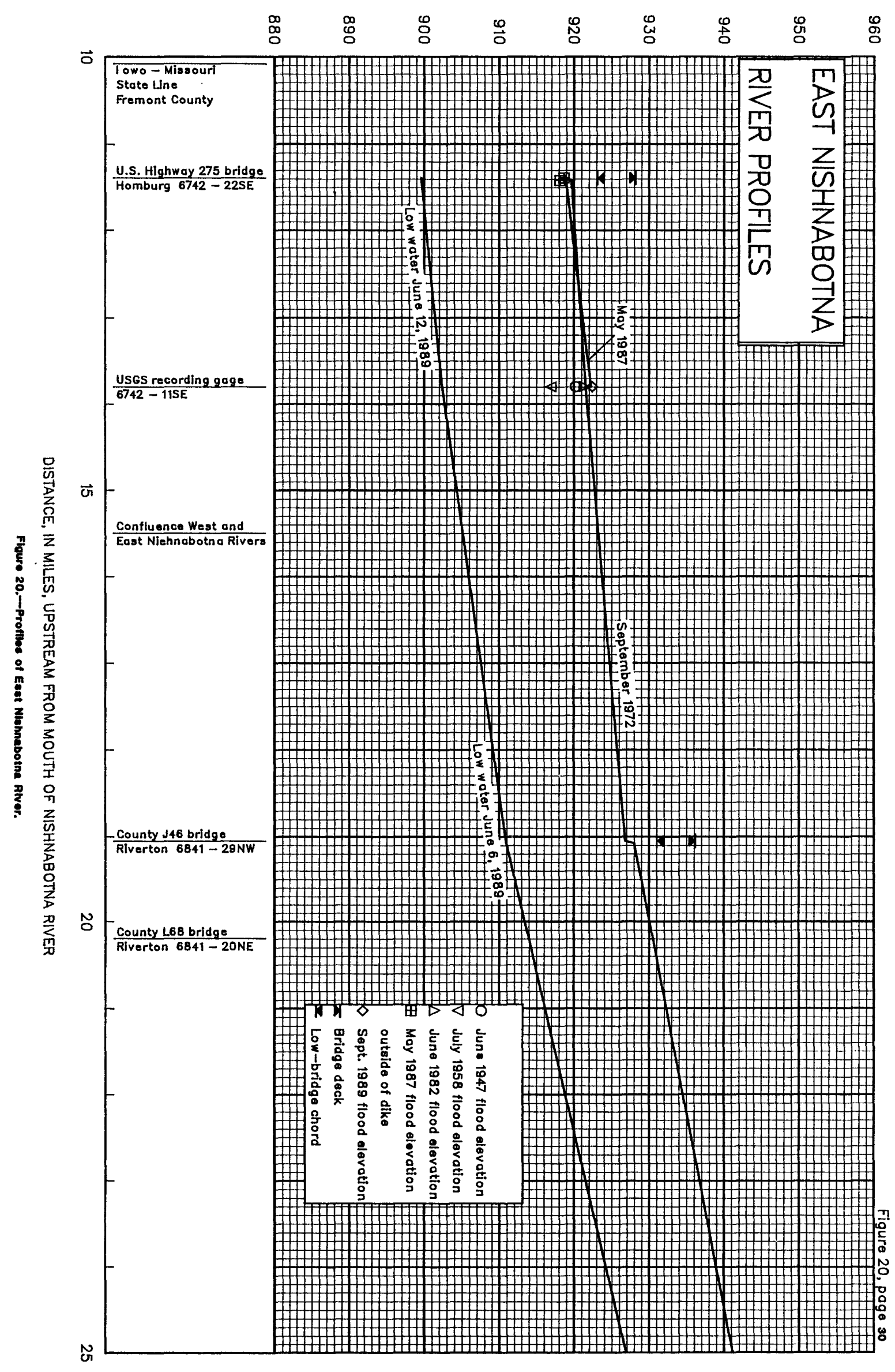


ELEVATION, IN FEET ABOVE SEA LEVEL

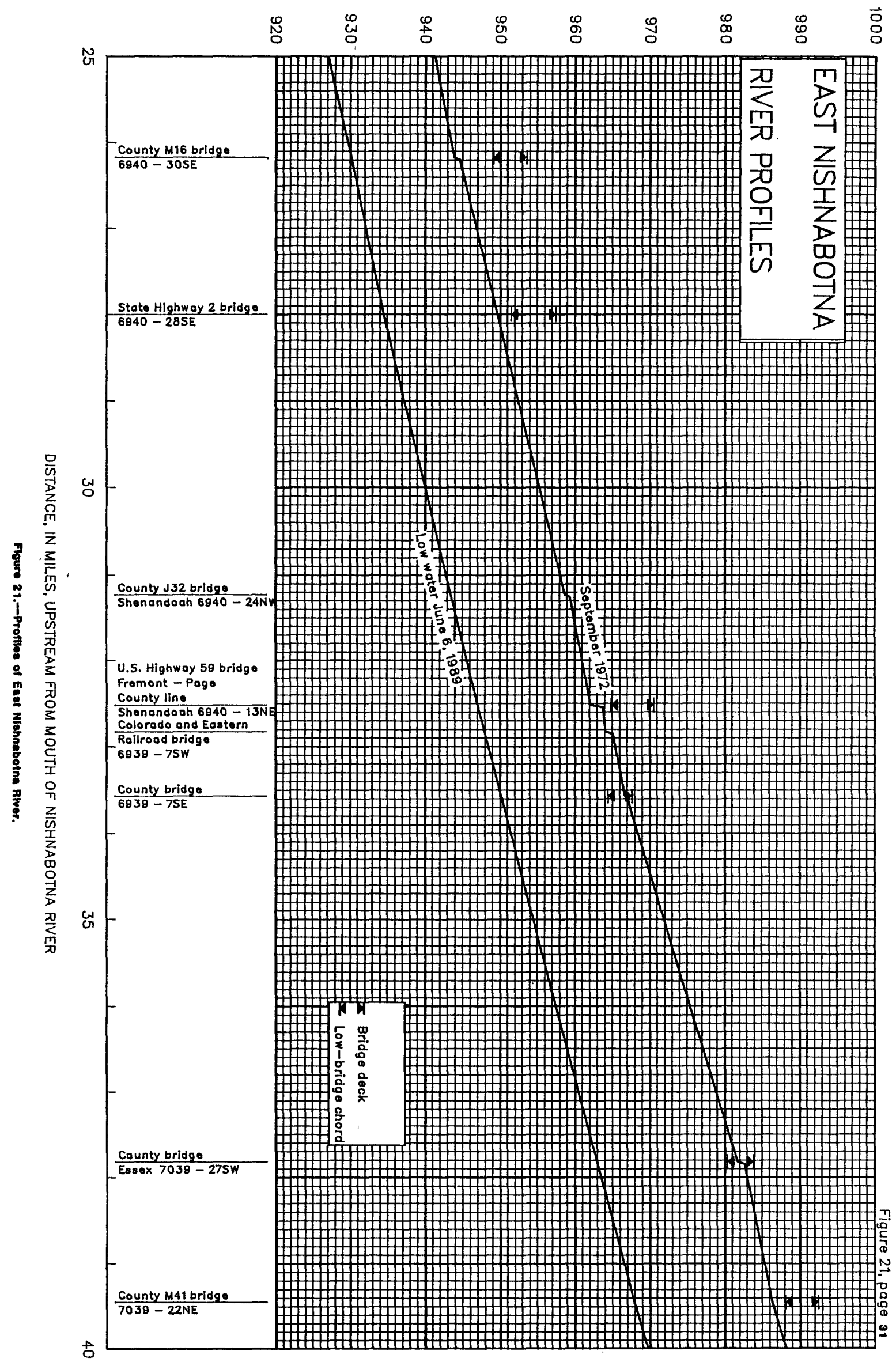


EL.EVATION, IN FEET ABOVE SEA LEVEL

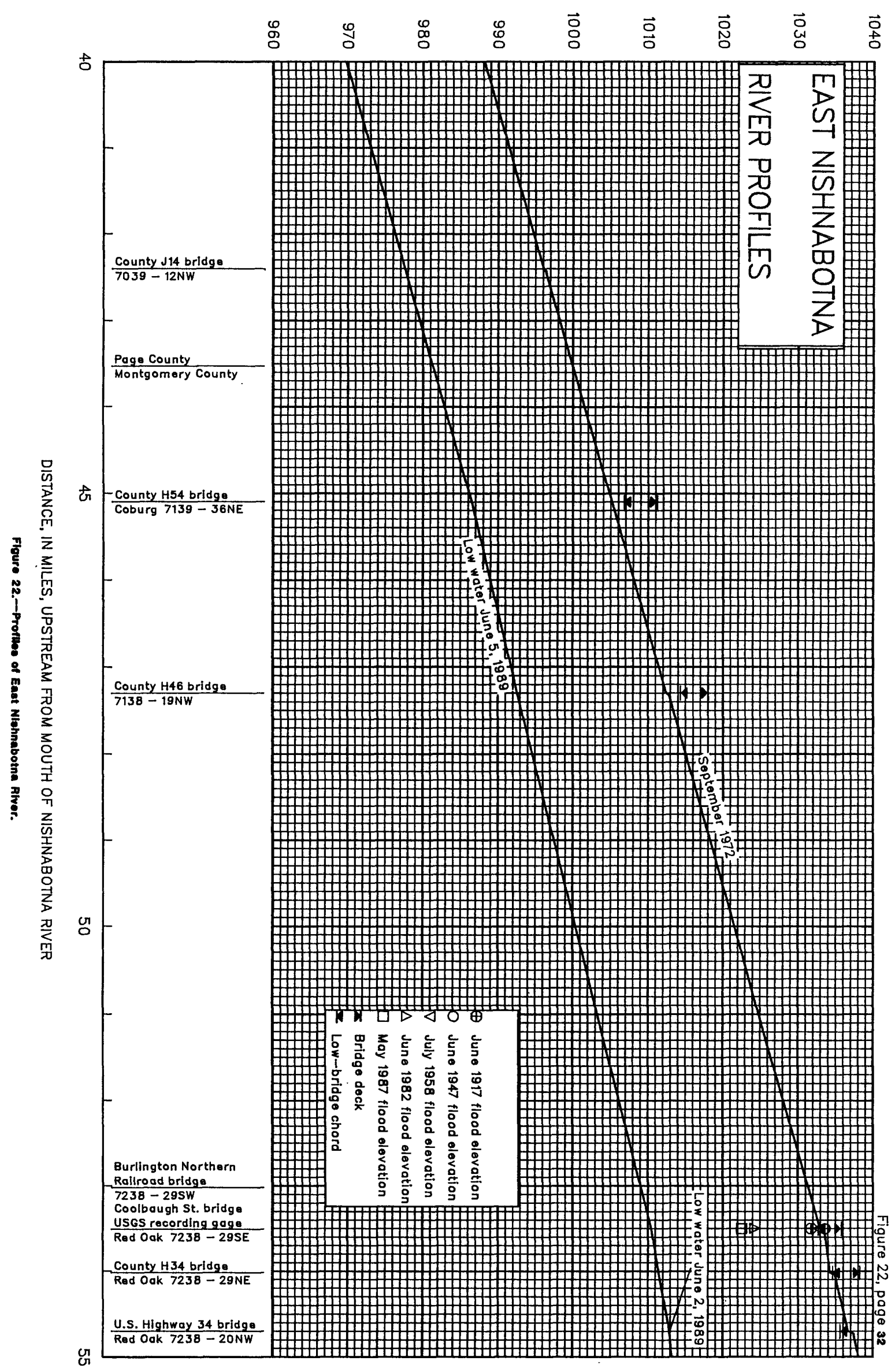


ELEVATION, IN FEET ABOVE SEA LEVEL

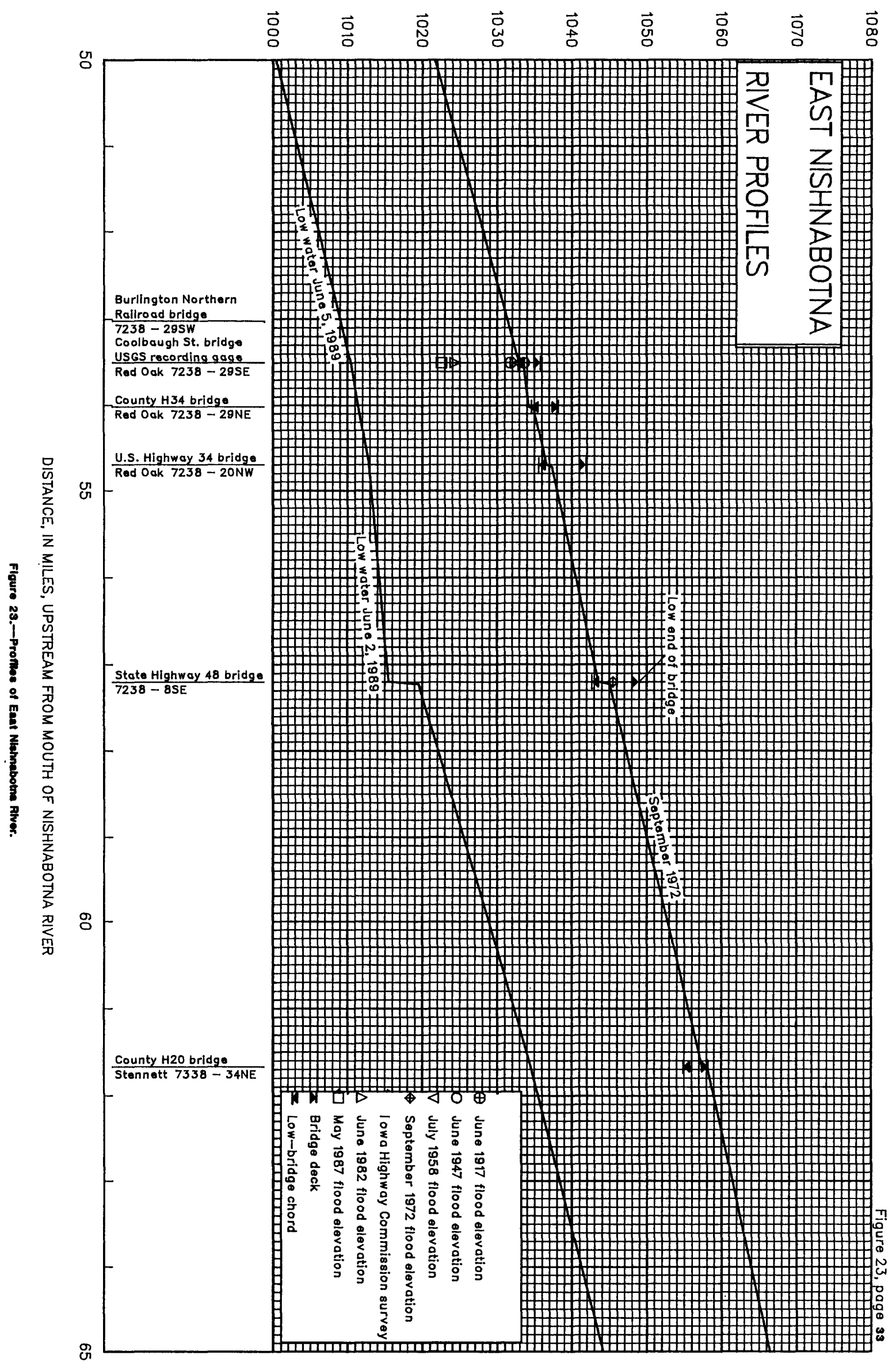




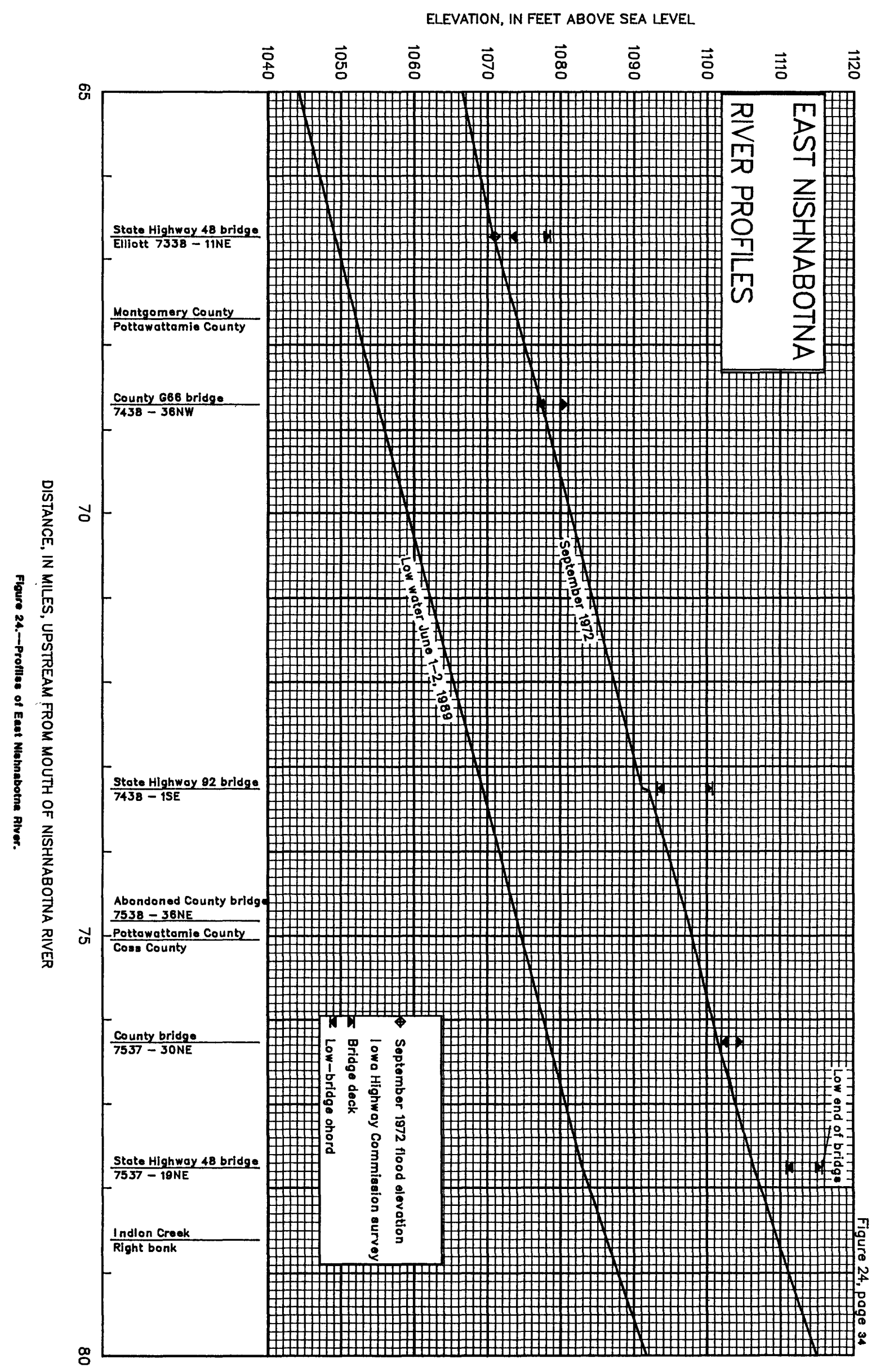


ELEVATION, IN FEET ABOVE SEA LEVEL

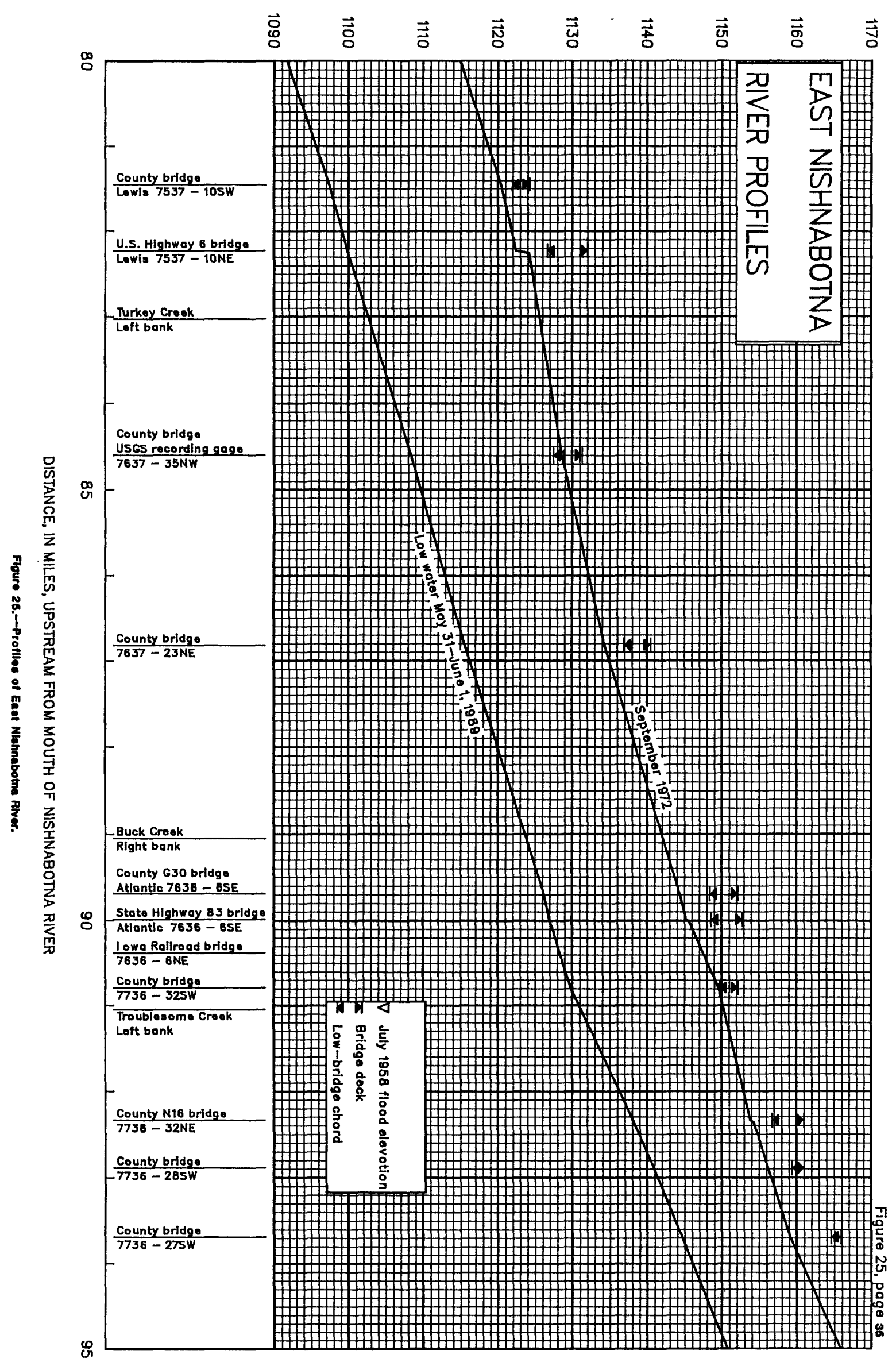




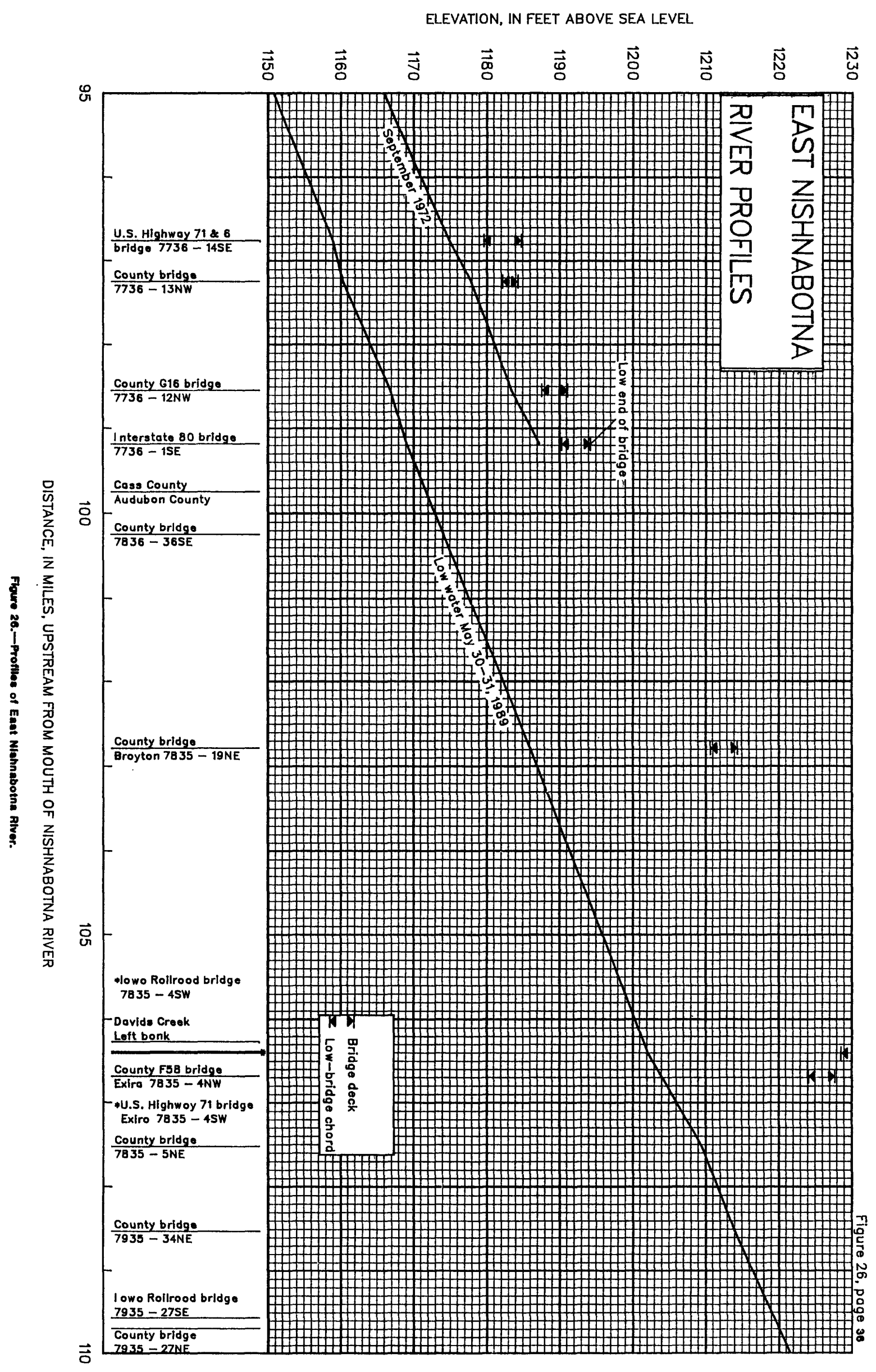


ELEVATION, IN FEET ABOVE SEA LEVEL

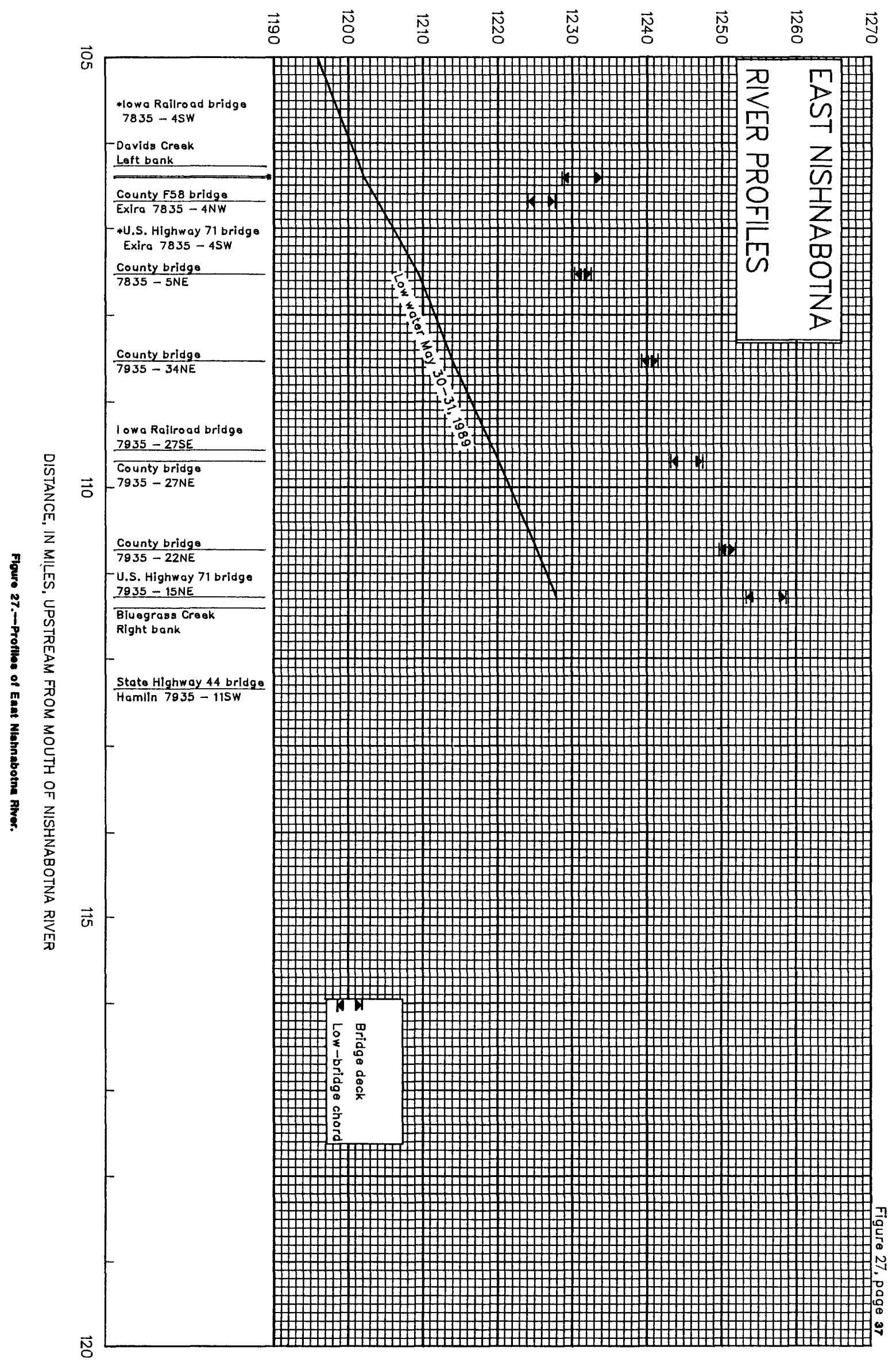


ELEVATION, IN FEET ABOVE SEA LEVEL.

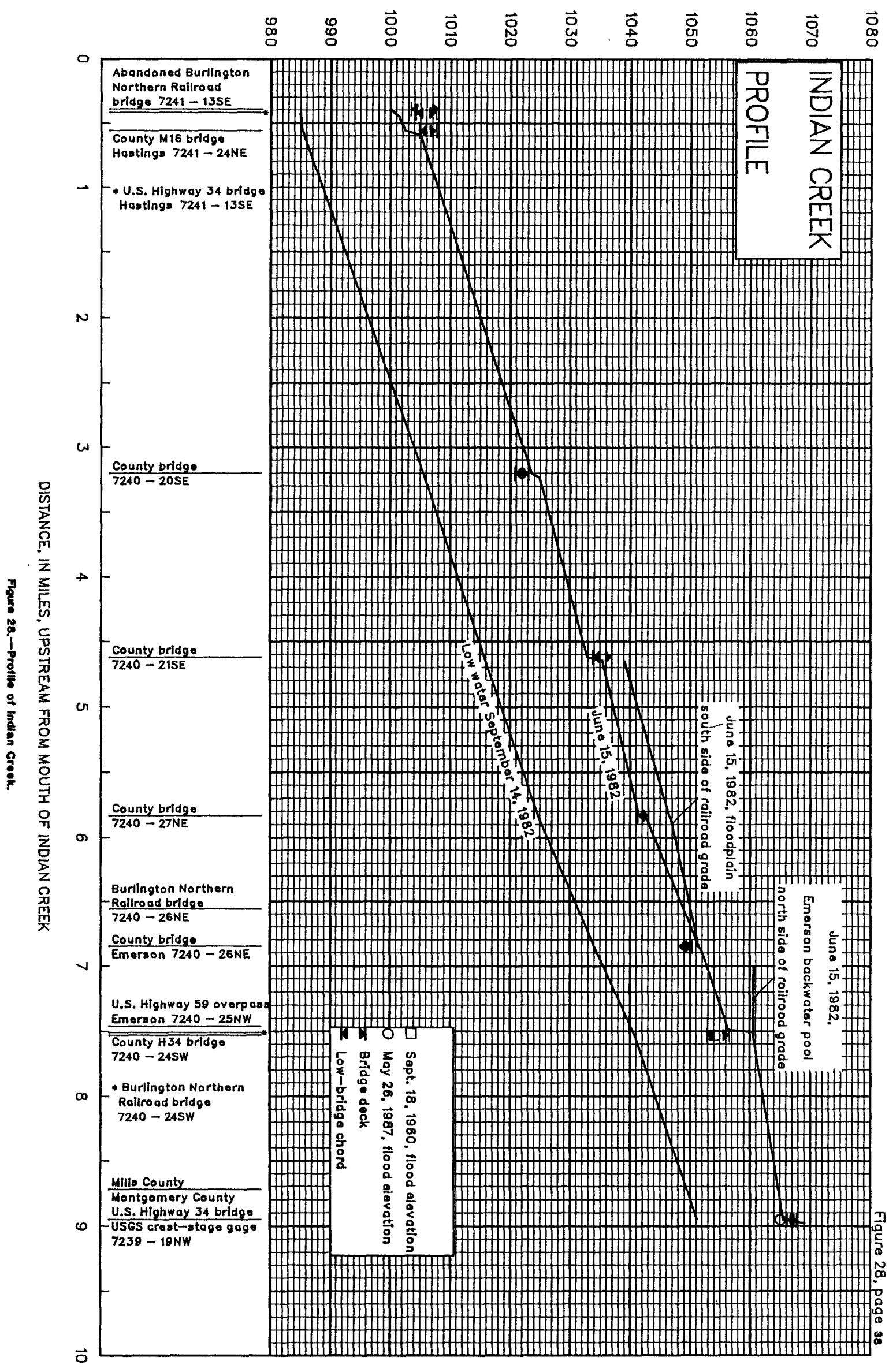


Table 3.--Peak stages and discharges for gaging stations,

\author{
Nishnabotna River basin, Iowa
}

The peak stage and discharge data for this report were compiled through september 30, 1988, for the 16 active and discontinued gaging stations located in the Nishnabotna River basin. The flood events, designated by calendar date, are in chronological order and grouped by water year (year ending September 30). In general, independent flood peaks above a pre-selected base (partial-duration series) are listed for the continuous record-gaging stations. The magnitude of the selected base discharge, given in the "Remarks" section of the headnote, was determined so that it would be equaled or exceeded on an average of about three times per year. Two flood peaks are considered independent if a plot of the recorded stages indicates a well-defined trough between the peaks, and if the instantaneous discharge of the trough is 25 percent or more below that of the lower peak (Novak, 1985, p. 93). Only the annual flood peaks are listed for the crest-stage gaging stations.

The gaging-station records are arranged in downstream order as explained in the annual streamflow reports of the U.S. Gelogical Survey (see references). Each gaging station is identified by a permanent number that is also used in figure 1 and in tables 1 and 4. The datum of the gage, when given, is sea level, formerly called sea Level Datum of 1929. Flood stage, as determined by the National Weather Service, is the stage at which overflow of the natural banks of the stream begins to cause damage in the reach in which the elevation is measured.

The following notations are used in the gaging station records:

1. A line in the "water year" column denotes a break or gap in the record of peaks.

2. A line beginning at the "date" column and continuing through the "discharge" column indicates a change in site and datum.

3. A line in the "date" and "discharge" columns indicates a change in site without a change in datum.

4. A line in the "gage height" column denotes a change in datum only.

The remainder of the information given is self-explanatory. 


\section{West Nishnabotna River at Harlan, Iowa (Discontinued September 30, 1982)}

Location.--Lat $41^{\circ} 38^{\prime} 41^{\prime \prime}$, long $95^{\circ} 18^{\prime} 50^{\prime \prime}$, in NW1/4 NE1/4 sec.19, T.79 N., R. $38 \mathrm{~W}$, Shelby County, Hydrologic Unit 10240002, in southeast part of City of Harlan, in city owned brick pumphouse on right bank, $50 \mathrm{ft}$ landward of levee, $250 \mathrm{ft}$ downstream from State Highway 44, $1.4 \mathrm{mi}$ downstream from mouth of West Fork, $80.1 \mathrm{mi}$ upstream from confluence with East Nishnabotna River, and $95.6 \mathrm{mi}$ upstream from mouth of Nishnabotna River.

Drainage area. --316 $\mathrm{mi}^{2}$.

Gage.--Water-stage recorder. Datum of gage is $1,162.89 \mathrm{ft}$ above sea level.

Stage-discharge relation.--Defined by current-meter measurements.

Flood stage.--Not determined.

Remarks.--Base for partial-duration series $1,500 \mathrm{ft}^{3} / \mathrm{s}$.

Peak stages and discharges

\begin{tabular}{|c|c|c|c|}
\hline $\begin{array}{l}\text { Water } \\
\text { year }\end{array}$ & Date & $\begin{array}{l}\text { Gage } \\
\text { height } \\
\text { (feet) }\end{array}$ & $\begin{array}{c}\text { Discharge } \\
\left(\mathrm{ft} \mathrm{t}^{3} / \mathrm{s}\right)\end{array}$ \\
\hline 1978 & $\begin{array}{lr}\text { Nov. } 9, & 1977 \\
\text { Mar. 21, } & 1978 \\
\text { May } 29, & 1978 \\
\text { Sept.13, } & 1978\end{array}$ & $\begin{array}{l}10.47 \\
18.88 \\
12.00 \\
26.18\end{array}$ & $\begin{array}{r}3,340 \\
7,790 \\
2,130 \\
14,500\end{array}$ \\
\hline 1979 & $\begin{array}{ll}\text { Mar. } 18, & 1979 \\
\text { Mar. } 23, & 1979 \\
\text { Mar. 30, } 1979 \\
\text { June 19, } 1979 \\
\text { Ju1y 30, } 1979 \\
\text { Sept. 6, } 1979\end{array}$ & $\begin{array}{l}24.03 \\
14.72 \\
11.74 \\
14.66 \\
16.59 a \\
10.60\end{array}$ & $\begin{array}{r}12,200 \\
4,040 \\
2,090 \\
4,000 \\
5,520 \\
1,500\end{array}$ \\
\hline 1980 & $\begin{array}{lr}\text { June } 6, & 1980 \\
\text { June } 15, & 1980\end{array}$ & $\begin{array}{l}17.02 \\
13.46\end{array}$ & $\begin{array}{l}5,870 \\
3,150\end{array}$ \\
\hline 1981 & Aug. $\quad 2,1981$ & 11.69 & 2,060 \\
\hline 1982 & $\begin{array}{l}\text { Feb. } 20,1982 \\
\text { Mar. } 19,1982 \\
\text { Aug. } 30,1982\end{array}$ & $\begin{array}{l}20.32 \\
18.90 \\
10.81\end{array}$ & $\begin{array}{l}8,490 \\
7,210 \\
1,600\end{array}$ \\
\hline
\end{tabular}

Discontinued September 30, 1982

a From high water mark. 
Location.--Lat $41^{\circ} 23^{\prime} 24^{\prime \prime}$, long $95^{\circ} 22^{\prime} 17^{\prime \prime}$, in NW1/4 NEl/4 sec. 18 ,

T.76N, R.39 W., Pottawattamie County, Hydrologic Unit 10240002 on right bank at upstream side of bridge on county road G30, $0.6 \mathrm{mi}$ west of Hancock school, $3.0 \mathrm{mi}$ downstream from Jim Creek, $59.6 \mathrm{mi}$ upstream from confluence with East Nishnabotna River, and $75.1 \mathrm{mi}$ upstream from mouth of Nishnabotna River.

Drainage area. $--609 \mathrm{mi}^{2}$.

Gage.--Water-stage encoder. Datum of gage is $1,085.83 \mathrm{ft}$ above sea leve1. Prior to Sept. 15, 1980, on downstream end of right pier at same datum.

Stage-discharge relation.--Defined by current-meter measurements .

Flood stage.--8 feet.

Remarks.--Base for partial-duration series, 4,000 $\mathrm{ft}^{3} / \mathrm{s}$.

Peak stages and discharges

\begin{tabular}{|c|c|c|c|}
\hline $\begin{array}{l}\text { Water } \\
\text { year }\end{array}$ & Date & $\begin{array}{l}\text { Gage } \\
\text { height } \\
\text { (feet) }\end{array}$ & $\begin{array}{c}\text { Discharge } \\
\left(\mathrm{ft} \mathrm{t}^{3} / \mathrm{s}\right)\end{array}$ \\
\hline 1960 & $\begin{array}{lr}\text { Mar. } & 29,1960 \\
\text { May } & 6,1960 \\
\text { Aug. } & 7,1960\end{array}$ & $\begin{array}{r}13.64 \\
12.57 \\
9.70\end{array}$ & $\begin{array}{l}9,320 \\
8,120 \\
4,800\end{array}$ \\
\hline 1961 & $\begin{array}{ll}\text { Mar. } & 14,1961 \\
\text { June } 27, & 1961 \\
\text { July } 28, & 1961\end{array}$ & $\begin{array}{r}10.31 \\
10.01 \\
9.69\end{array}$ & $\begin{array}{l}5,430 \\
4,460 \\
4,160\end{array}$ \\
\hline 1962 & $\begin{array}{lll}\text { Mar. } & 22, & 1962 \\
\text { May } & 21, & 1962 \\
\text { May } 26, & 1962 \\
\text { May } & 29, & 1962 \\
\text { June } & 10, & 1962\end{array}$ & $\begin{array}{l}10.90 \\
12.10 \\
11.58 \\
13.50 \\
11.18\end{array}$ & $\begin{array}{l}6,680 \\
7,420 \\
6,800 \\
9,240 \\
6,200\end{array}$ \\
\hline 1963 & $\begin{array}{ll}\text { Mar. } 11, & 1963 \\
\text { Mar. } 14, & 1963\end{array}$ & $\begin{array}{l}9.75 \\
8.98\end{array}$ & $\begin{array}{l}4,860 \\
4,100\end{array}$ \\
\hline 1964 & $\begin{array}{lrl}\text { Apr. } & 2, & 1964 \\
\text { May } & 8, & 1964 \\
\text { May } & 26, & 1964 \\
\text { June } & 22, & 1964 \\
\text { July } & 3, & 1964 \\
\text { July } & 7, & 1964 \\
\text { July } & 11, & 1964\end{array}$ & $\begin{array}{r}9.20 \\
9.55 \\
14.50 \\
10.92 \\
10.10 \\
9.65 \\
12.34\end{array}$ & $\begin{array}{r}4,010 \\
5,320 \\
10,500 \\
6,090 \\
5,210 \\
4,700 \\
7,760\end{array}$ \\
\hline
\end{tabular}


06807410 West Nishnabotna River at Hancock, Iowa--(Continued)

Peak stages and discharges

\begin{tabular}{|c|c|c|c|}
\hline $\begin{array}{l}\text { Water } \\
\text { year }\end{array}$ & Date & $\begin{array}{l}\text { Gage } \\
\text { height } \\
\text { (feet) }\end{array}$ & $\begin{array}{c}\text { Discharge } \\
\left(\mathrm{ft}^{3} / \mathrm{s}\right)\end{array}$ \\
\hline 1965 & $\begin{array}{lr}\text { Mar. 1, } 1965 \\
\text { Mar. 17, } 1965 \\
\text { Mar. 31, } 1965 \\
\text { Apr. } 5,1965 \\
\text { May 26, } 1965 \\
\text { Sept. 28, } 1965\end{array}$ & $\begin{array}{r}20.44 \text { a } \\
15.39 \\
13.94 \\
10.91 \\
11.49 \\
8.85\end{array}$ & $\begin{array}{l}18,000 \mathrm{~b} \\
12,000 \\
13,100 \\
8,070 \\
8,940 \\
5,160\end{array}$ \\
\hline 1966 & June 12, 1966 & 9.07 & 4,170 \\
\hline 1967 & $\begin{array}{l}\text { June } 7,1967 \\
\text { June } 12,1967 \\
\text { June 16, } 1967 \\
\text { June 19, } 1967 \\
\text { June 24, } 1967 \\
\text { June 28, } 1967\end{array}$ & $\begin{array}{r}11.05 \\
9.09 \\
14.16 \\
8.88 \\
8.71 \\
8.58\end{array}$ & $\begin{array}{r}6,260 \\
4,830 \\
10,900 \\
4,620 \\
4,450 \\
4,320\end{array}$ \\
\hline 1968 & June 25,1968 & 6.41 & 2,380 \\
\hline 1969 & $\begin{array}{lrr}\text { Mar. } & 18, & 1969 \\
\text { Mar. } & 24, & 1969 \\
\text { July } & 9, & 1969\end{array}$ & $\begin{array}{r}15.02 \\
9.98 \\
11.38\end{array}$ & $\begin{array}{r}12,600 \\
5,040 \\
6,830\end{array}$ \\
\hline 1970 & 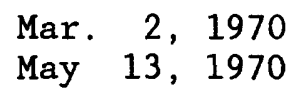 & $\begin{array}{l}9.56 \\
9.89\end{array}$ & $\begin{array}{l}4,580 \\
4,930\end{array}$ \\
\hline 1971 & $\begin{array}{lrl}\text { Feb. } & 19, & 1971 \\
\text { Mar. } & 14, & 1971 \\
\text { June } & 6, & 1971\end{array}$ & $\begin{array}{l}18.56 \\
10.98 \\
10.28\end{array}$ & $\begin{array}{r}17,700 \\
6,730 \\
5,610\end{array}$ \\
\hline 1972 & $\begin{array}{l}\text { Feb. 29, } 1972 \\
\text { Sept.13, } 1972\end{array}$ & $\begin{array}{r}8.81 \\
22.12\end{array}$ & $\begin{array}{r}4,210 \\
26,400\end{array}$ \\
\hline 1973 & $\begin{array}{lr}\text { Dec. } 30, & 1972 \\
\text { Jan. } 17,1973 \\
\text { Feb. 24, } 1973 \\
\text { Mar. 1, } 1973 \\
\text { May } 7,1973 \\
\text { July 4, } 1973 \\
\text { Sept. 26, } 1973\end{array}$ & $\begin{array}{r}9.47 \\
11.86 \\
8.63 \\
9.43 \\
12.07 \\
11.90 \\
16.54\end{array}$ & $\begin{array}{r}5,070 \\
7,270 \\
4,120 \\
4,920 \\
7,800 \\
7,780 \\
14,300\end{array}$ \\
\hline 1974 & $\begin{array}{lll}\text { Oct. } & 11, & 1973 \\
\text { May } & 16, & 1974 \\
\text { May } & 19, & 1974\end{array}$ & $\begin{array}{r}9.13 \\
9.10 \\
12.81\end{array}$ & $\begin{array}{l}4,730 \\
4,700 \\
8,950\end{array}$ \\
\hline
\end{tabular}


06807410 West Nishnabotna River at Hancock, Iowa--(Continued)

Peak stages and discharges

\begin{tabular}{|c|c|c|c|}
\hline $\begin{array}{l}\text { Water } \\
\text { year }\end{array}$ & Date & $\begin{array}{l}\text { Gage } \\
\text { height } \\
\text { (feet) }\end{array}$ & $\begin{array}{c}\text { Discharge } \\
\left(\mathrm{ft}^{3} / \mathrm{s}\right)\end{array}$ \\
\hline 1975 & $\begin{array}{cl}\text { Apr. } 28, & 1975 \\
\text { June } 23, & 1975 \\
\text { June } 25, & 1975 \\
\text { Aug. } 29, & 1975\end{array}$ & $\begin{array}{l}14.13 \\
10.42 \\
10.85 \\
10.47\end{array}$ & $\begin{array}{r}10,700 \\
6,110 \\
6,580 \\
6,170\end{array}$ \\
\hline 1976 & June 13, 1976 & 12.70 & 8,810 \\
\hline 1977 & Sept. 1, 1977 & 4.70 & 1,110 \\
\hline 1978 & $\begin{array}{ll}\text { Mar. 21, } & 1978 \\
\text { Apr. 8, } & 1978 \\
\text { Sept. 13, } & 1978\end{array}$ & $\begin{array}{r}13.82 \\
8.57 \\
17.85\end{array}$ & $\begin{array}{r}10,500 \\
4,330 \\
16,800\end{array}$ \\
\hline 1979 & $\begin{array}{ll}\text { Mar. } 13,1979 \\
\text { Mar. } 18,1979 \\
\text { Mar. 23, } 1979 \\
\text { July } 30,1979\end{array}$ & $\begin{array}{r}10.44 \\
19.54 \\
9.45 \\
10.73\end{array}$ & $\begin{array}{r}6,130 \\
18,300 \\
5,000 \\
6,210\end{array}$ \\
\hline 1980 & $\begin{array}{l}\text { June } 6,1980 \\
\text { June } 15,1980\end{array}$ & $\begin{array}{l}11.22 \\
14.01\end{array}$ & $\begin{array}{l}6,630 \\
9,880\end{array}$ \\
\hline 1981 & May $\quad 4,1981$ & 6.75 & 2,510 \\
\hline 1982 & $\begin{array}{ll}\text { Feb. } 21, & 1982 \\
\text { Mar. } 19, & 1982\end{array}$ & $\begin{array}{l}15.84 \\
13.94\end{array}$ & $\begin{array}{l}13,900 \\
11,200\end{array}$ \\
\hline 1983 & Feb. 20, 1983 & 11.70 & 8,310 \\
\hline 1984 & $\begin{array}{cc}\text { Apr. } & 30,1984 \\
\text { June } 13,1984 \\
\text { June } 15,1984 \\
\text { June } 16,1984\end{array}$ & $\begin{array}{r}10.55 \\
9.57 \\
14.52 \\
13.28\end{array}$ & $\begin{array}{r}6,840 \\
5,170 \\
12,000 \\
10,300\end{array}$ \\
\hline 1985 & $\begin{array}{ll}\text { Dec. } & 16,1984 \\
\text { Dec. } 28, & 1984 \\
\text { Feb. } 21, & 1985\end{array}$ & $\begin{array}{r}8.62 \\
9.48 \\
14.49 \mathrm{a}\end{array}$ & $\begin{array}{c}4,700 \\
5,610 \\
c\end{array}$ \\
\hline 1986 & $\begin{array}{lr}\text { Feb. } 26,1986 \\
\text { Mar. } 18,1986 \\
\text { May } 10,1986 \\
\text { June } 14,1986 \\
\text { July } 9,1986 \\
\text { July } 10,1986 \\
\text { July } 14,1986\end{array}$ & $\begin{array}{r}12.37 \\
11.55 \\
15.19 \\
12.15 \\
8.11 \\
8.26 \\
8.83\end{array}$ & $\begin{array}{r}9,100 \\
8,020 \\
13,000 \\
8,800 \\
4,140 \\
4,290 \\
4,910\end{array}$ \\
\hline
\end{tabular}


06807410 West Nishnabotna River at Hancock, Iowa--(Continued)

Peak stages and discharges

\begin{tabular}{|c|c|c|c|}
\hline $\begin{array}{l}\text { Water } \\
\text { year }\end{array}$ & Date & $\begin{array}{c}\text { Gage } \\
\text { height } \\
\text { (feet) }\end{array}$ & $\begin{array}{c}\text { Discharge } \\
\left(\mathrm{ft}^{3} / \mathrm{s}\right)\end{array}$ \\
\hline 1987 & $\begin{array}{l}\text { Oct. } 11,1986 \\
\text { Aug. } 26,1987\end{array}$ & $\begin{array}{r}12.39 \\
9.97\end{array}$ & $\begin{array}{l}7,510 \\
4,870\end{array}$ \\
\hline 1988 & $\begin{array}{lr}\text { Jan. } & 30,1988 \\
\text { June } & 8,1988\end{array}$ & $\begin{array}{r}10.63 \mathrm{a} \\
7.45\end{array}$ & $\begin{array}{c}c \\
2,730\end{array}$ \\
\hline
\end{tabular}


Location. - - Lat $41^{\circ} 13^{\prime} 57^{\prime \prime}$, long $95^{\circ} 22^{\prime} 51^{\prime \prime}$, in NW1/4 sec. 7, T.74 N., R. 39 W., Pottawattamie County, at bridge on State Highway 92, 2 mi east of Carson.

Drainage area. $--45.9 \mathrm{mi}^{2}$.

Gage.--Crest-stage gage.

Stage-discharge relation.--Undefined due to channel degradation.

Remarks.--Only annual peaks are shown.

Peak stages and discharges

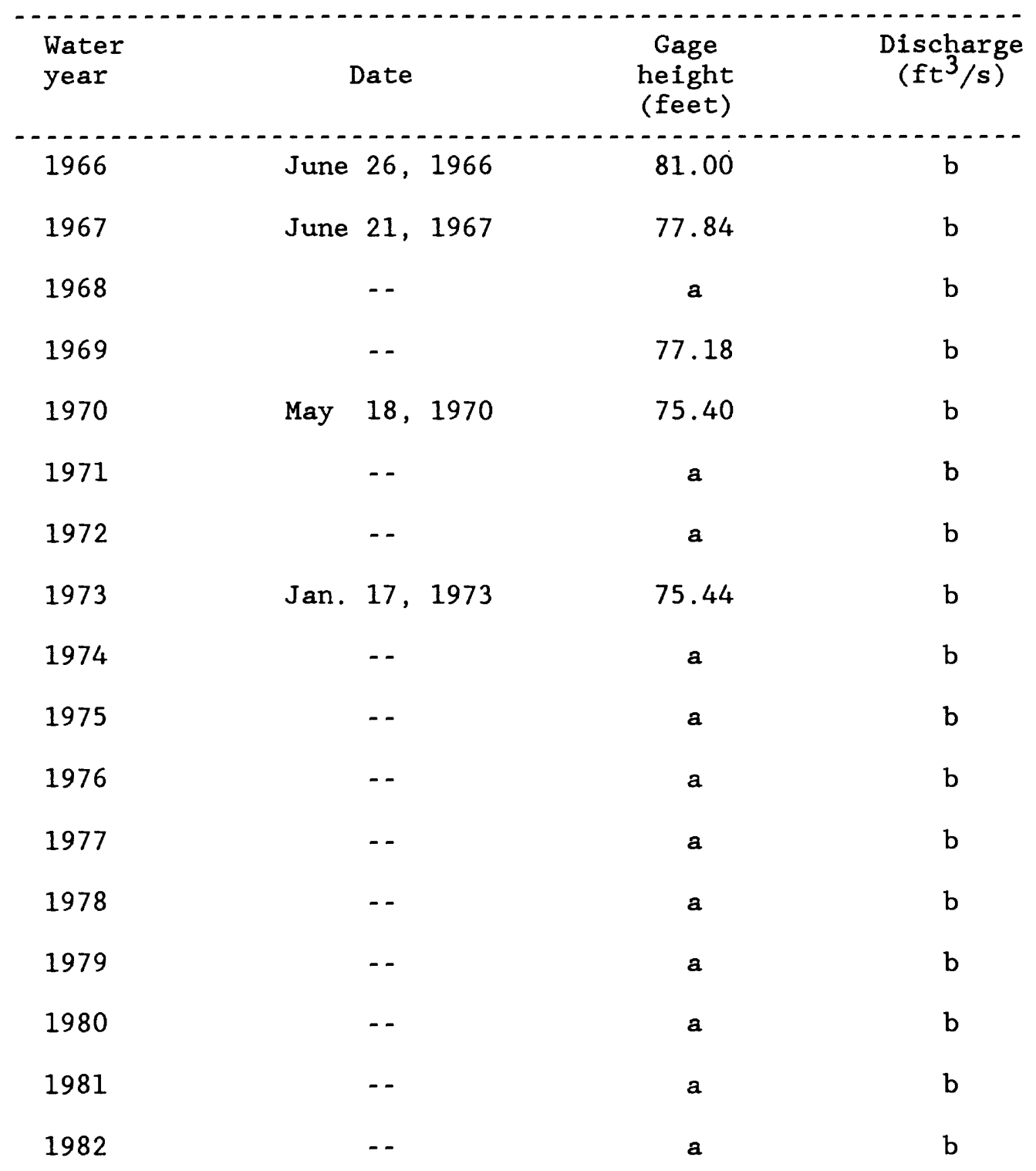


06807418 Graybill Creek near Carson, Iowa--(Continued)

Peak stages and discharges

\begin{tabular}{|c|c|c|c|}
\hline $\begin{array}{l}\text { Water } \\
\text { year }\end{array}$ & Date & $\begin{array}{c}\text { Gage } \\
\text { height } \\
\text { (feet) }\end{array}$ & $\begin{array}{c}\text { Discharge } \\
\left(\mathrm{ft}^{3} / \mathrm{s}\right)\end{array}$ \\
\hline 1983 & - & a & $\mathrm{b}$ \\
\hline 1984 & $\cdots$ & $a$ & $\mathrm{~b}$ \\
\hline 1985 & -- & a & $\mathrm{b}$ \\
\hline 1986 & $\cdots$ & a & $\mathrm{b}$ \\
\hline 1987 & Aug. $\quad 8,1987$ & 76.12 & b \\
\hline 1988 & -- & a & $\mathrm{b}$ \\
\hline
\end{tabular}

a Peak stage did not reach bottom of gage.

b Discharge not determined. 
06807470 Indian Creek near Emerson, Iowa

Location.--Lat $41^{\circ} 01^{\prime} 50^{\prime \prime}$, long $95^{\circ} 22^{\prime} 51^{\prime \prime}$, in NW1/4 sec. 19, T.72 N., R.39 W., Montgomery County, at bridge on U.S. Highway 34, $1 \mathrm{mi}$ east of Emerson.

Drainage area.--37.3 $\mathrm{mi}^{2}$.

Gage.--Crest-stage gage.

Stage-discharge relation.--Defined by current-meter measurements and an indirect measurement.

Remarks.--Only annual peaks are shown.

Peak stages and discharges

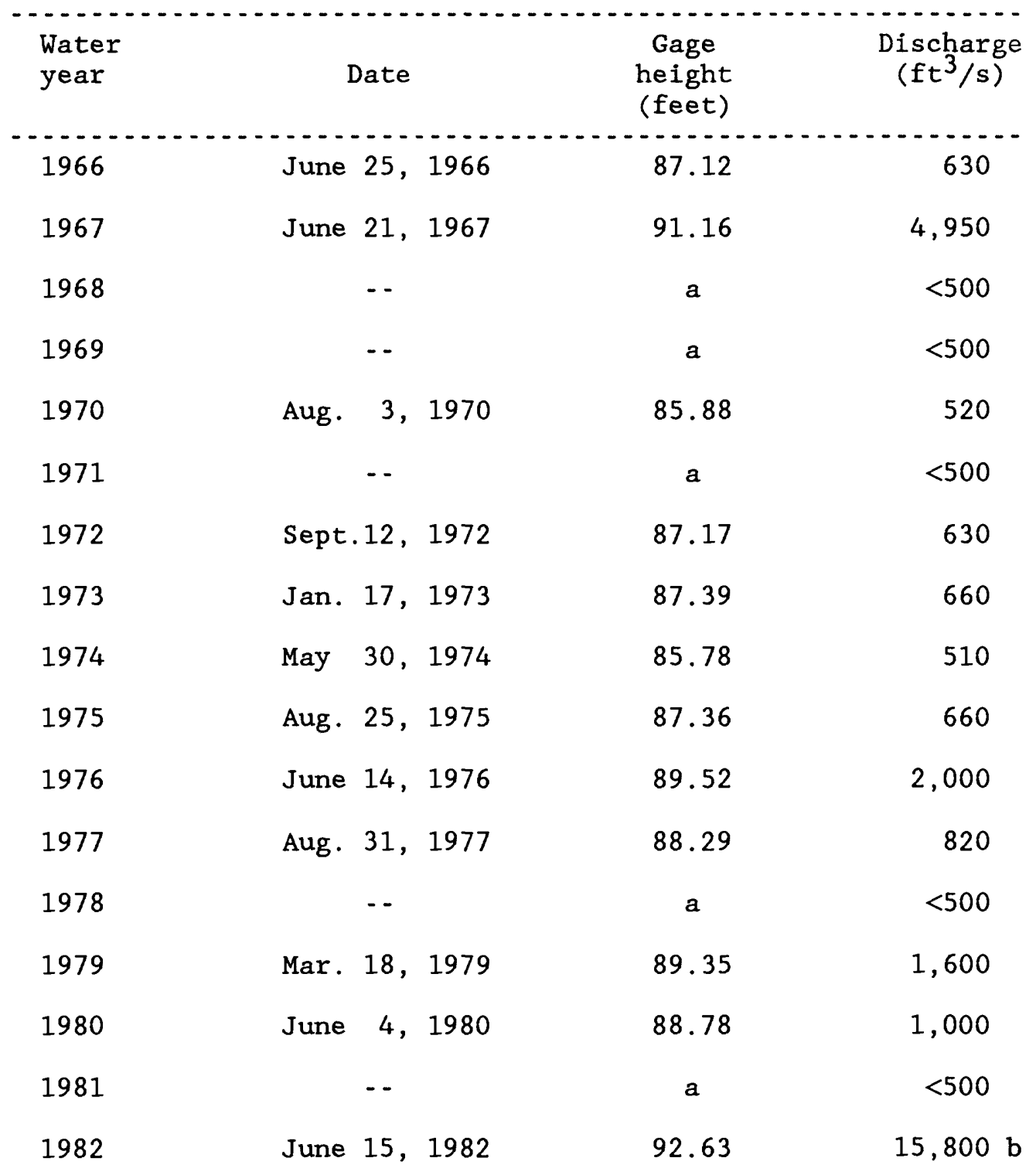


06807470 Indian Creek near Emerson, Iowa--(Continued)

Peak stages and discharges

\begin{tabular}{|c|c|c|c|}
\hline $\begin{array}{l}\text { Water } \\
\text { year }\end{array}$ & Date & $\begin{array}{c}\text { Gage } \\
\text { height } \\
\text { (feet) }\end{array}$ & $\begin{array}{c}\text { Discharge } \\
\left(\mathrm{ft}^{3} / \mathrm{s}\right)\end{array}$ \\
\hline 1983 & $\cdots$ & a & $<500$ \\
\hline 1984 & June 15,1984 & 87.87 & 1,310 \\
\hline 1985 & -- & a & $594 \mathrm{c}$ \\
\hline 1986 & April 4, 1986 & 86.73 & 1,080 \\
\hline 1987 & May 26,1987 & 91.78 & 9,600 \\
\hline 1988 & -- & a & $<500 \mathrm{c}$ \\
\hline
\end{tabular}

a Peak stage did not reach bottom of gage.

b Discharge computed from an indirect measurement.

c Reconstructed discharge based on regional analysis of streamflow and rainfall data.

$<$ Less than. 
06807500 West Nishnabotna River at White Cloud, Iowa

(Discontinued June 21, 1924)

Location.--Lat $40^{\circ} 57^{\prime}$, long $95^{\circ} 34^{\prime}$, in sec.2, T.71 N., R.41 W, Mills County, at highway bridge, 3 miles above mouth of Silver Creek, near Chicago, Burlington \& Quincy Railroad and Wabash Railway crossing at White Cloud, and 4 miles southeast of Malvern.

Drainage area. $--967 \mathrm{mi}^{2}$.

Gage.--Chain gage read twice daily.

Stage-discharge relation.--Defined by current meter measurements.

Flood stage. - - Not determined.

Remarks.--Only annual peaks are shown.

Peak stages and discharges

\begin{tabular}{|c|c|c|c|}
\hline $\begin{array}{l}\text { Water } \\
\text { year }\end{array}$ & Date & $\begin{array}{c}\text { Gage } \\
\text { height } \\
\text { (feet) }\end{array}$ & $\begin{array}{c}\text { Discharge } \\
\left(\mathrm{ft}^{3} / \mathrm{s}\right)\end{array}$ \\
\hline 1919 & Oct. 28,1918 & -- & 7,500 \\
\hline 1920 & Apr. 19,1920 & 18.00 & 9,130 \\
\hline 1921 & Sept. 20, 1921 & 17.61 & 8,710 \\
\hline 1922 & July 30,1922 & 19.4 & 10,600 \\
\hline 1923 & Aug. 11,1923 & 17.6 & 8,710 \\
\hline 1924 & June 8,1924 & 17.0 & 8,080 \\
\hline
\end{tabular}

Discontinued June 21, 1924 
06807720 Middle Silver Creek near Avoca, Iowa

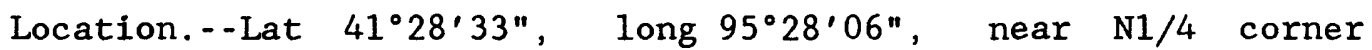
sec.17, T. 77 N., R.40 W., Pottawattamie County, at bridge on State Highway $83,7 \mathrm{mi}$ west of Avoca.

Drainage area.--3.21 $\mathrm{mi}^{2}$.

Gage.--Crest-stage gage.

Stage-discharge relation.--Defined by step-backwater computations and an indirect measurement at $642 \mathrm{ft}^{3} / \mathrm{s}$.

Remarks.--Only annual peaks are shown.

Peak stages and discharges

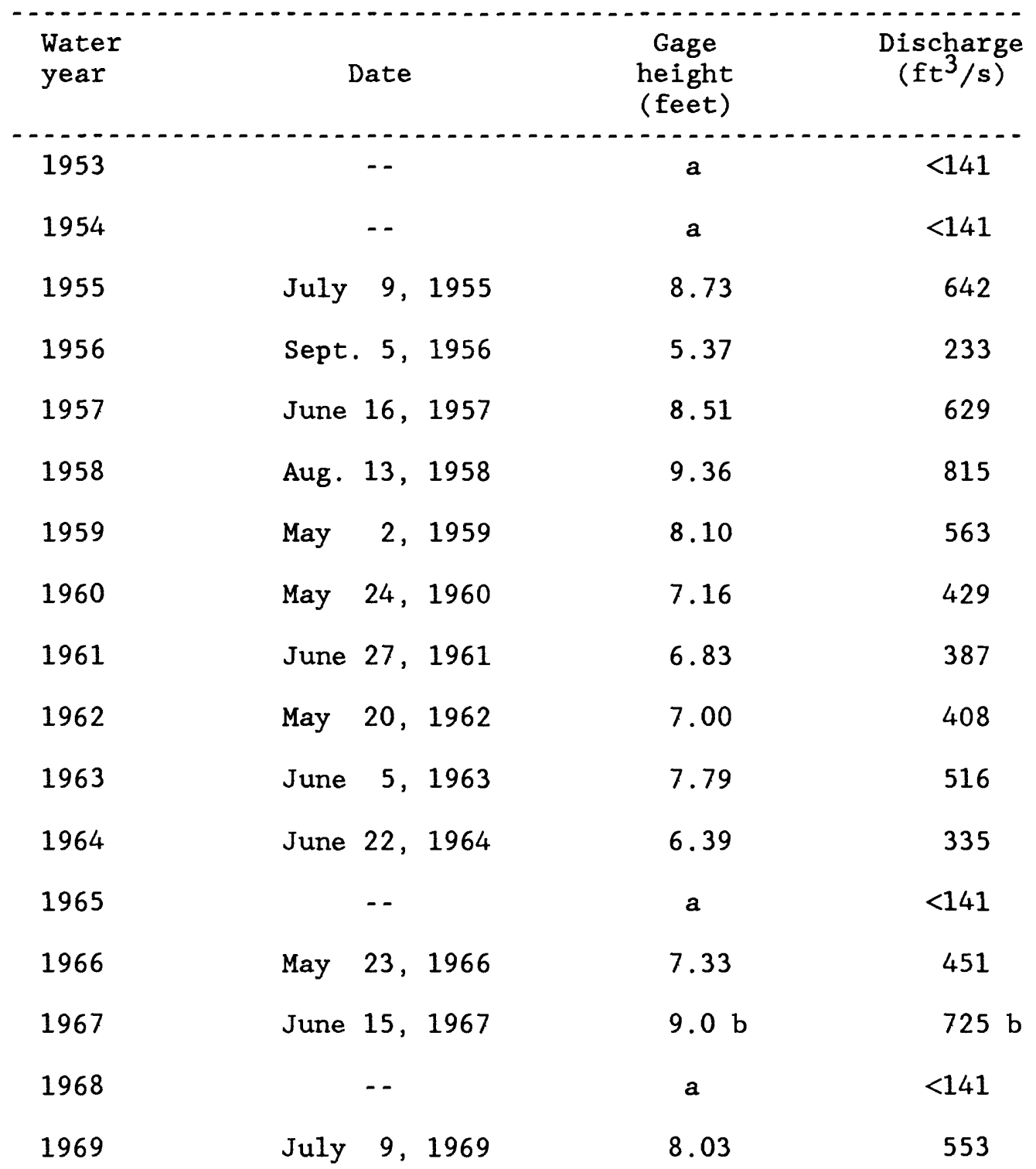


06807720 Middle Silver Creek near Avoca, Iowa--(Continued)

Peak stages and discharges

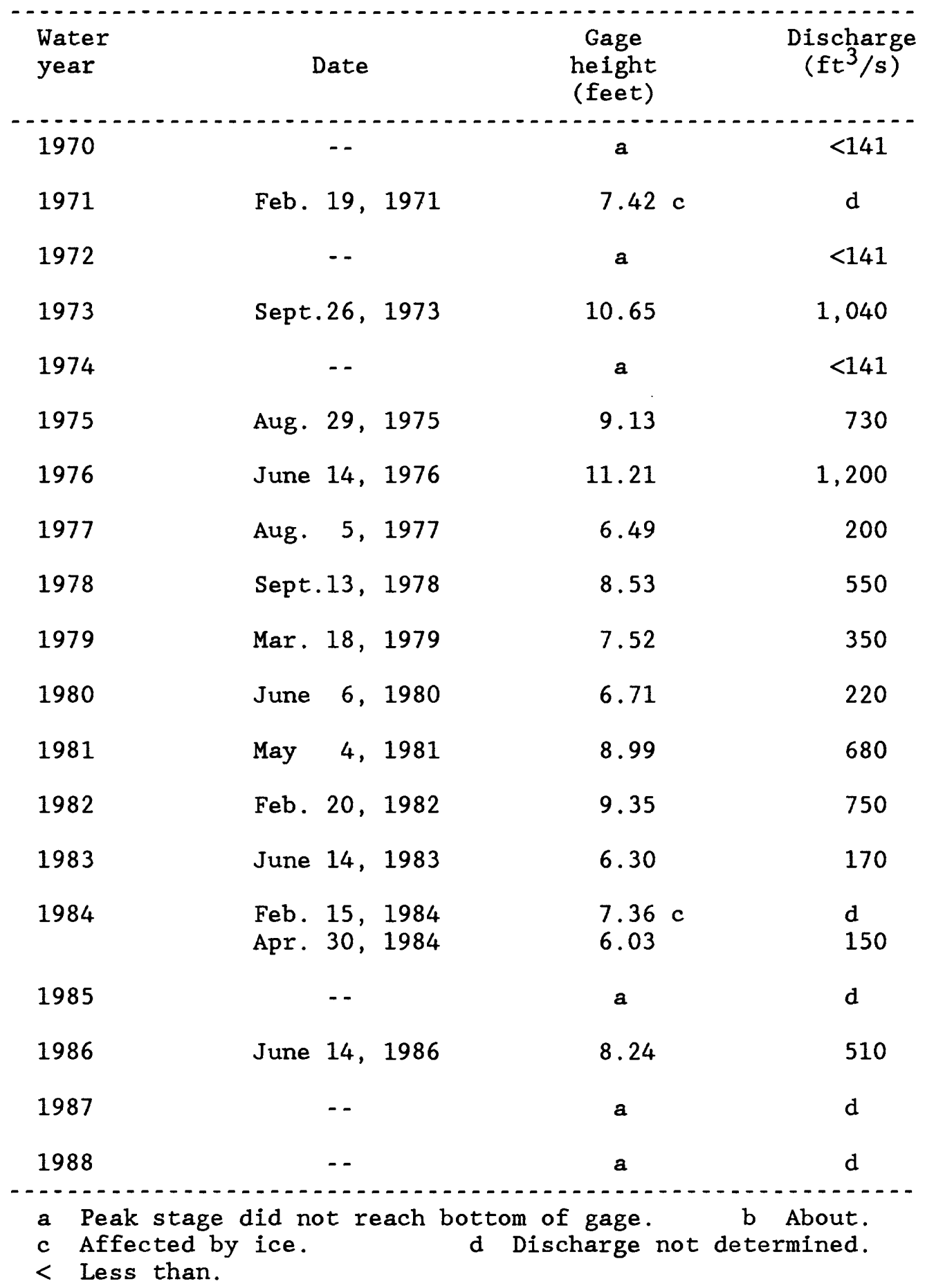




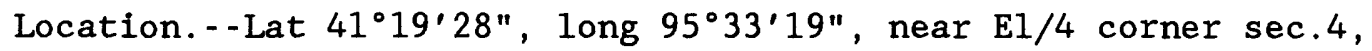
T.75 N., R.41 W., Pottawattamie County, at bridge on county road, and $8.5 \mathrm{mi}$ northwest of Oakland.

Drainage area. $--25.7 \mathrm{mi}^{2}$.

Gage.--Crest-stage gage.

Stage-discharge relation.--Defined by current-meter and indirect measurements.

Remarks.--Only annual peaks are shown.

Peak stages and discharges

\begin{tabular}{|c|c|c|c|c|}
\hline $\begin{array}{l}\text { Water } \\
\text { year }\end{array}$ & & Date & $\begin{array}{c}\text { Gage } \\
\text { height } \\
\text { (feet) }\end{array}$ & $\begin{array}{c}\text { Discharge } \\
\left(\mathrm{ft}^{3} / \mathrm{s}\right)\end{array}$ \\
\hline 1953 & June & 10,1953 & 9.53 & 991 \\
\hline 1954 & Aug. & 22,1954 & 8.27 & 759 \\
\hline 1955 & July & 9, 1955 & 9.94 & 1,120 \\
\hline 1956 & Aug. & 17,1956 & 7.56 & 631 \\
\hline 1957 & June & 16,1957 & 9.81 & 1,040 \\
\hline 1958 & Sept. & . 5,1958 & 12.28 & 1,450 \\
\hline 1959 & May & 28,1959 & 9.24 & 1,030 \\
\hline 1960 & May & 25,1960 & 8.15 & 898 \\
\hline 1961 & June & 12,1961 & 7.15 & 778 \\
\hline 1962 & July & 13,1962 & 5.55 & 586 \\
\hline 1963 & June & 5, 1963 & 6.55 & 706 \\
\hline 1964 & & -- & a & $<396$ \\
\hline 1965 & June & 29,1965 & 8.31 & 917 \\
\hline 1966 & June & 26,1966 & 7.79 & 855 \\
\hline 1967 & June & 15,1967 & 11.26 & 1,300 \\
\hline 1968 & May & 15,1968 & 6.79 & 735 \\
\hline 1969 & & -- & 7.56 & 700 \\
\hline
\end{tabular}




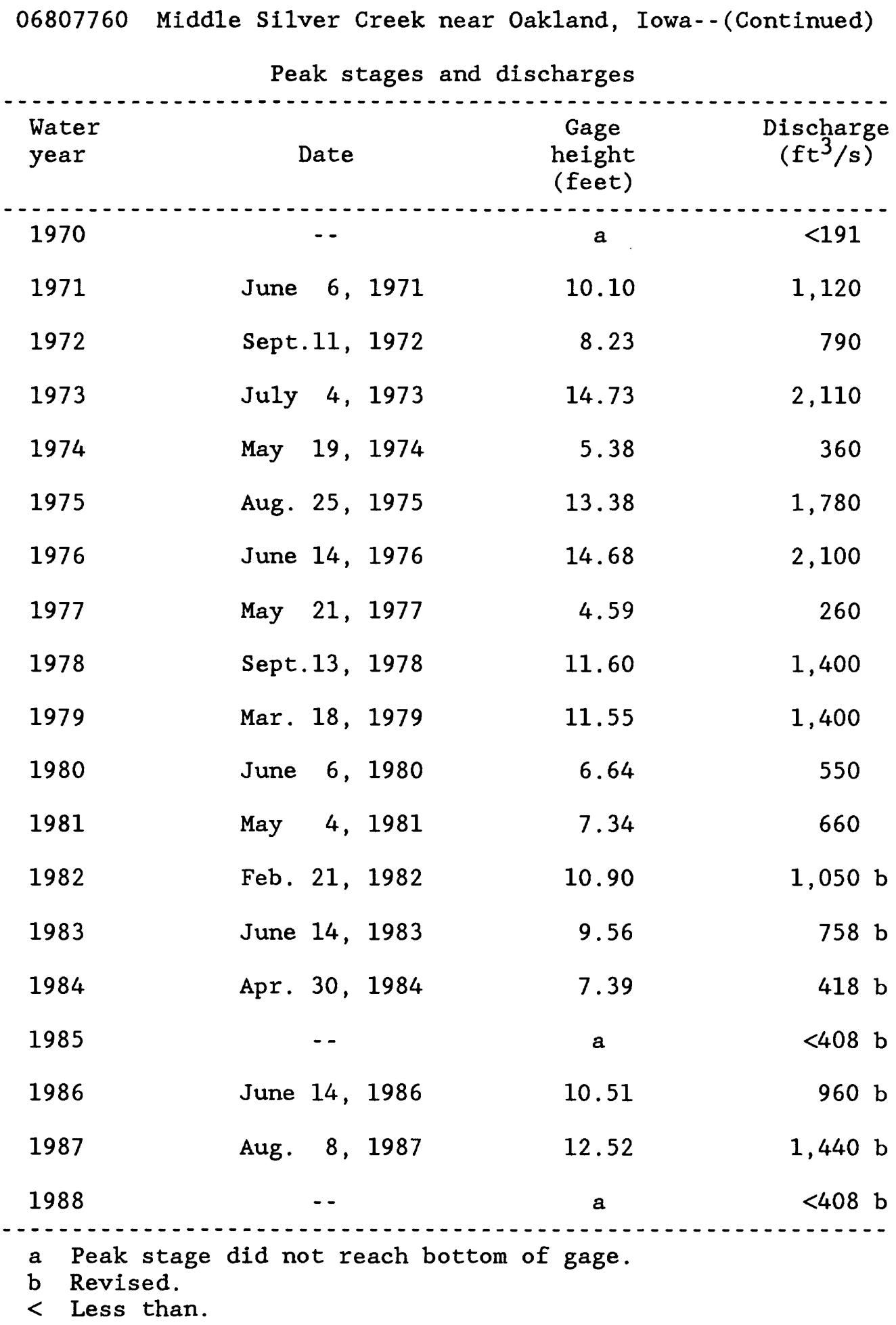


06807780 Middle Silver Creek at Treynor, Iowa

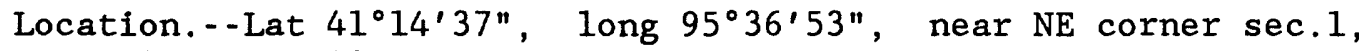
T.74 N., R.42 W., Pottawattamie County, at bridge on county road L55, $1 \mathrm{mi}$ north of Treynor.

Drainage area. $--42.7 \mathrm{mi}^{2}$.

Gage.--Crest-stage gage.

Stage-discharge relation.--Defined by current-meter and indirect measurements.

Remarks.--Only annual peaks are shown.

Peak stages and discharges

\begin{tabular}{|c|c|c|c|}
\hline $\begin{array}{l}\text { Water } \\
\text { year }\end{array}$ & Date & $\begin{array}{c}\text { Gage } \\
\text { height } \\
\text { (feet) }\end{array}$ & $\begin{array}{c}\text { Discharge } \\
\left(\mathrm{ft}^{3} / \mathrm{s}\right)\end{array}$ \\
\hline 1953 & June 10,1953 & 12.28 & 1,700 \\
\hline 1954 & June 21,1954 & 9.80 & 1,180 \\
\hline 1955 & Mar. 2, 1955 & 10.32 & 1,280 \\
\hline 1956 & July 11, 1956 & $9.04 \mathrm{a}$ & $911 \mathrm{~b}$ \\
\hline 1957 & June 16,1957 & 10.01 & 1,220 \\
\hline 1958 & Sept. 5, 1958 & 16.01 & 2,600 \\
\hline 1959 & June 28, 1959 & 11.89 & 1,620 \\
\hline 1960 & Aug. 28, 1960 & 13.84 & 2,060 \\
\hline 1961 & Feb. 22, 1961 & 9.84 & 1,320 \\
\hline 1962 & July 27, 1962 & 9.00 & 1,020 \\
\hline 1963 & June 5,1963 & 8.26 & 1,110 \\
\hline 1964 & June 22, 1964 & 8.82 & 1,320 \\
\hline 1965 & June 29, 1965 & 9.24 & 1,390 \\
\hline 1966 & June 26, 1966 & 15.82 & 2,560 \\
\hline 1967 & June 7,1967 & 10.98 & 1,600 \\
\hline 1968 & May 15,1968 & 5.41 & 735 \\
\hline 1969 & July 17, 1969 & 9.96 & 1,330 \\
\hline
\end{tabular}




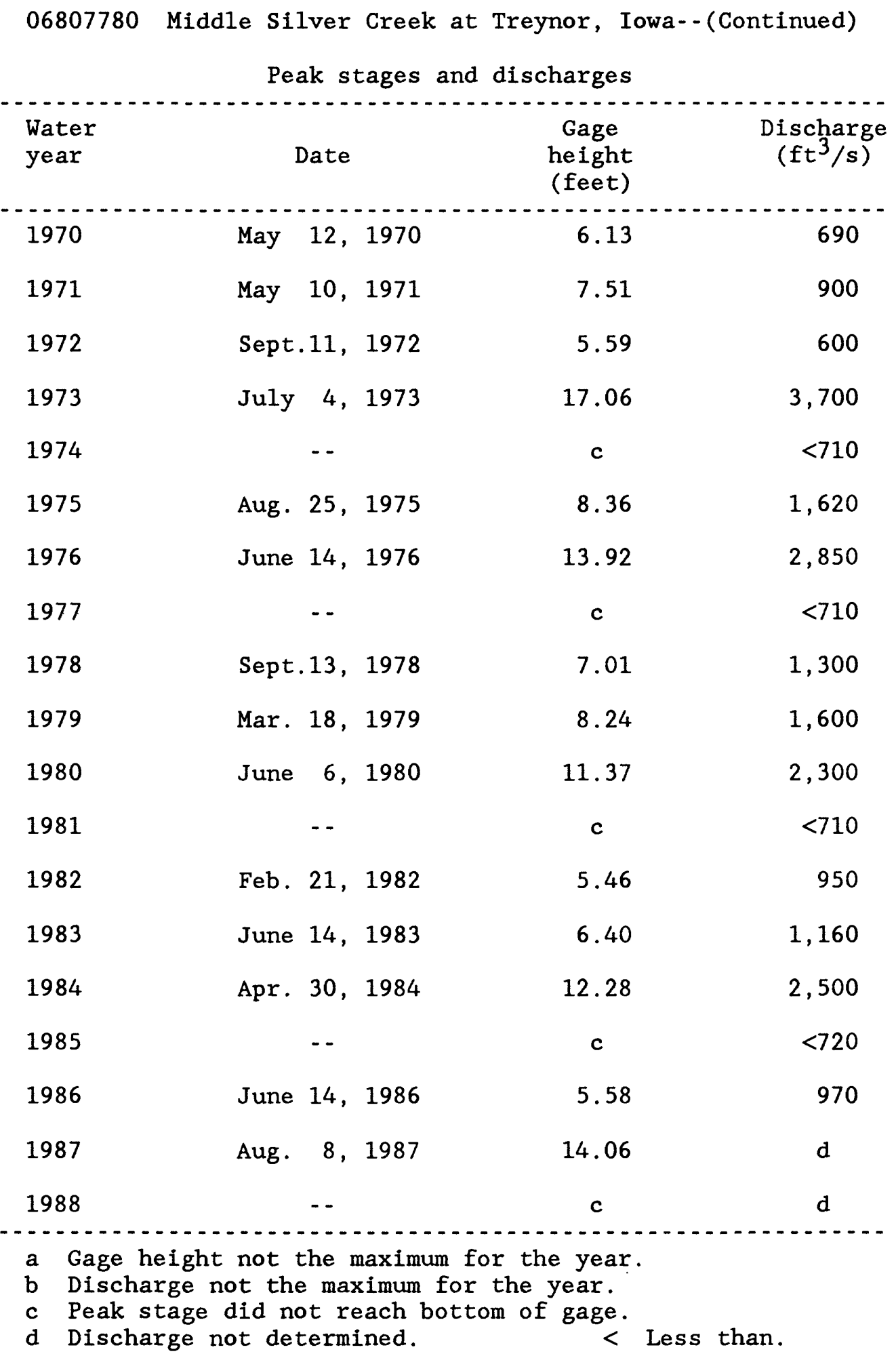


06808000 Mule Creek near Malvern, Iowa

(Discontinued Sept. 30, 1969)

Location.--Lat 40 56'36", long $95^{\circ} 35^{\prime} 42^{\prime \prime}$, in NE1/4 NE1/4 sec.19, T.71 N., R.41 W., Milis County, on right bank $170 \mathrm{ft}$ upstream from culvert on county road L63, $0.2 \mathrm{mi}$ downstream from unnamed tributary, $1.8 \mathrm{mi}$ upstream from mouth, and $4.3 \mathrm{mi}$ south of Malvern.

Drainage area. $--10.6 \mathrm{mi}^{2}$.

Gage.--Water-stage recorder. Datum of gage is $974.20 \mathrm{ft}$ above sea level (levels by Soil Conservation Service). Prior to 0ct. 1, 1964, water-stage recorder at site $180 \mathrm{ft}$ downstream. Oct. 1 , 1964, to Mar. 25, 1965, nonrecording gage with supplemental water-stage recorder at site 180 feet downstream. Mar. 26 to July 13, 1965, nonrecording gages at various locations. July 14,1965 to Sept. 30, 1969, water-stage recorder at site 170 ft upstream of culvert at same datum.

Stage-discharge relation.--Defined by current-meter measurements below $510 \mathrm{ft}^{3} / \mathrm{s}$ and extended above on basis of slope-area measurement of peak flow.

Remarks.--Base for partial-duration series, $300 \mathrm{ft}^{3} / \mathrm{s}$. Basin became partially regulated by the construction of 24 impoundment dams during 1954-56.

Peak stages and discharges

\begin{tabular}{|c|c|c|c|}
\hline $\begin{array}{l}\text { Water } \\
\text { year }\end{array}$ & Date & $\begin{array}{l}\text { Gage } \\
\text { height } \\
\text { (feet) }\end{array}$ & $\begin{array}{c}\text { Discharge } \\
\left(\mathrm{ft}^{3} / \mathrm{s}\right)\end{array}$ \\
\hline 1954 & $\begin{array}{l}\text { Aug. } 21,1954 \\
\text { Aug. } 23,1954\end{array}$ & $\begin{array}{l}15.84 \\
14.19\end{array}$ & $\begin{array}{l}2,070 \text { a } \\
1,680\end{array}$ \\
\hline 1955 & Mar. 1, 1955 & 7.51 & 411 \\
\hline 1956 & $\begin{array}{ll}\text { July } 11, & 1956 \\
\text { July } 15, & 1956 \\
\text { Aug. } & 18,1956\end{array}$ & $\begin{array}{l}7.55 \\
15.54 \\
10.4\end{array}$ & $\begin{array}{r}398 \\
1,990 \\
867\end{array}$ \\
\hline 1957 & $\begin{array}{lr}\text { May } 29, & 1957 \\
\text { June } 7, & 1957 \\
\text { June } 15, & 1957 \\
\text { June } 17, & 1957 \\
\text { July } 1, & 1957\end{array}$ & $\begin{array}{r}12.58 \\
9.80 \\
6.30 \\
7.40 \\
8.70\end{array}$ & $\begin{array}{r}1,310 \\
849 \\
334 \\
479 \\
678\end{array}$ \\
\hline 1958 & 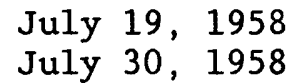 & $\begin{array}{r}6.92 \\
12.35\end{array}$ & $\begin{array}{r}334 \\
1,180\end{array}$ \\
\hline
\end{tabular}


06808000 Mule Creek near Malvern, Iowa--(Continued)

Peak stages and discharges

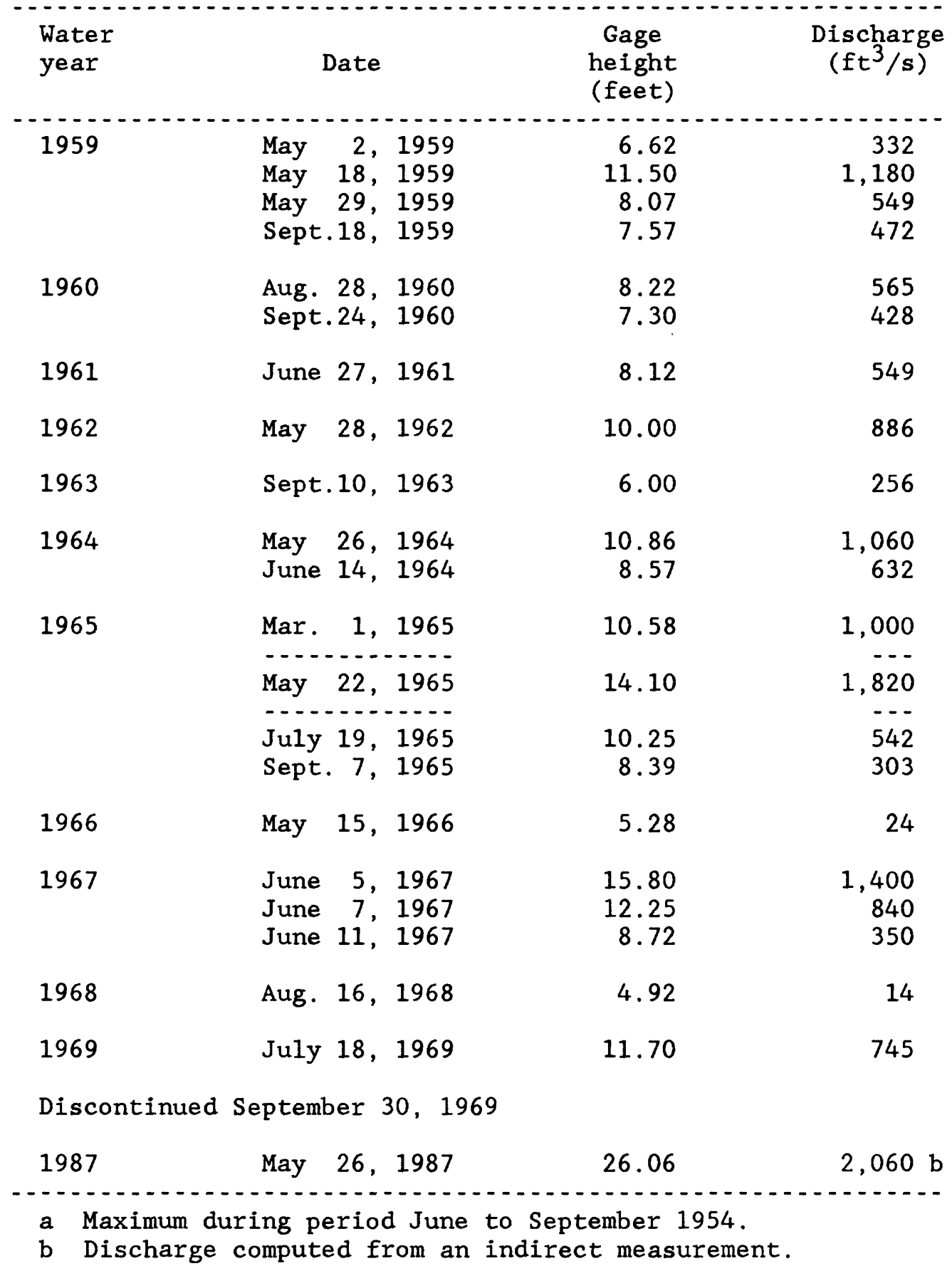


06808200 Spring Valley Creek near Tabor, Iowa (Discontinued Sept. 30, 1964)

Location.--Lat 40 $54^{\prime} 35^{\prime \prime}$, long $95^{\circ} 36^{\prime} 00^{\prime \prime}$, in SW1/4NE1/4 sec. 31 , T.71 N., R.41 W., Mills County, on left bank $20 \mathrm{ft}$ downstream from county road bridge, $1.5 \mathrm{mi}$ upstream from mouth, and 4.0 mi northeast of Tabor.

Drainage area. $--7.65 \mathrm{mi}^{2}$.

Gage.-Water-stage recorder and concrete control. Altitude of gage is $975 \mathrm{ft}$ (from topographic map).

Stage-discharge relation.--Defined by current-meter measurements below $210 \mathrm{ft}^{3} / \mathrm{s}$ and above by slope-area measurement.

Remarks.--Base for partial-duration series, $250 \mathrm{ft}^{3} / \mathrm{s}$.

Peak stages and discharges

\begin{tabular}{|c|c|c|c|}
\hline $\begin{array}{l}\text { Water } \\
\text { year }\end{array}$ & Date & $\begin{array}{l}\text { Gage } \\
\text { height } \\
\text { (feet) }\end{array}$ & $\begin{array}{c}\text { Discharge } \\
\left(\mathrm{ft}^{3} / \mathrm{s}\right)\end{array}$ \\
\hline 1956 & $\begin{array}{ll}\text { June } 11, & 1956 \\
\text { July } 15, & 1956 \\
\text { Aug. } 18, & 1956\end{array}$ & $\begin{array}{r}8.59 \\
13.50 \\
8.78\end{array}$ & $\begin{array}{r}317 \\
2,310 \\
359\end{array}$ \\
\hline 1957 & $\begin{array}{lrl}\text { May } & 29, & 1957 \\
\text { June } & 7, & 1957 \\
\text { June } 17, & 1957 \\
\text { July } 1, & 1957\end{array}$ & $\begin{array}{r}12.07 \\
10.58 \\
8.90 \\
10.33\end{array}$ & $\begin{array}{r}1,480 \\
865 \\
380 \\
778\end{array}$ \\
\hline 1958 & $\begin{array}{ll}\text { July } 19, & 1958 \\
\text { July } 30, & 1958\end{array}$ & $\begin{array}{l}10.85 \\
15.48\end{array}$ & $\begin{array}{r}935 \\
4,150\end{array}$ \\
\hline 1959 & $\begin{array}{lrl}\text { May } 18, & 1959 \\
\text { May } 29, & 1959 \\
\text { Aug. } 5, & 1959 \\
\text { Sept. } 18, & 1959\end{array}$ & $\begin{array}{c}12.57 \\
10.0 \\
8.40 \\
8.51\end{array}$ & $\begin{array}{c}1,730 \\
670 \mathrm{~b} \\
279 \\
298\end{array}$ \\
\hline 1960 & $\begin{array}{ll}\text { Aug. } 17, & 1960 \\
\text { Aug. } 28, & 1960 \\
\text { Sept. } 24, & 1960\end{array}$ & $\begin{array}{r}9.30 \\
12.28 \\
8.63\end{array}$ & $\begin{array}{r}473 \\
1,570 \\
328\end{array}$ \\
\hline 1961 & June 27,1961 & 12.13 & 1,480 \\
\hline 1962 & $\begin{array}{lrl}\text { May } & 28, & 1962 \\
\text { May } & 31, & 1962 \\
\text { June } & 6, & 1962\end{array}$ & $\begin{array}{r}11.77 \\
9.36 \\
8.85 \text { a }\end{array}$ & $\begin{array}{r}1,300 \\
486 \\
370 \mathrm{~b}\end{array}$ \\
\hline 1963 & Sept.10, 1963 & 12.13 & 1,480 \\
\hline
\end{tabular}




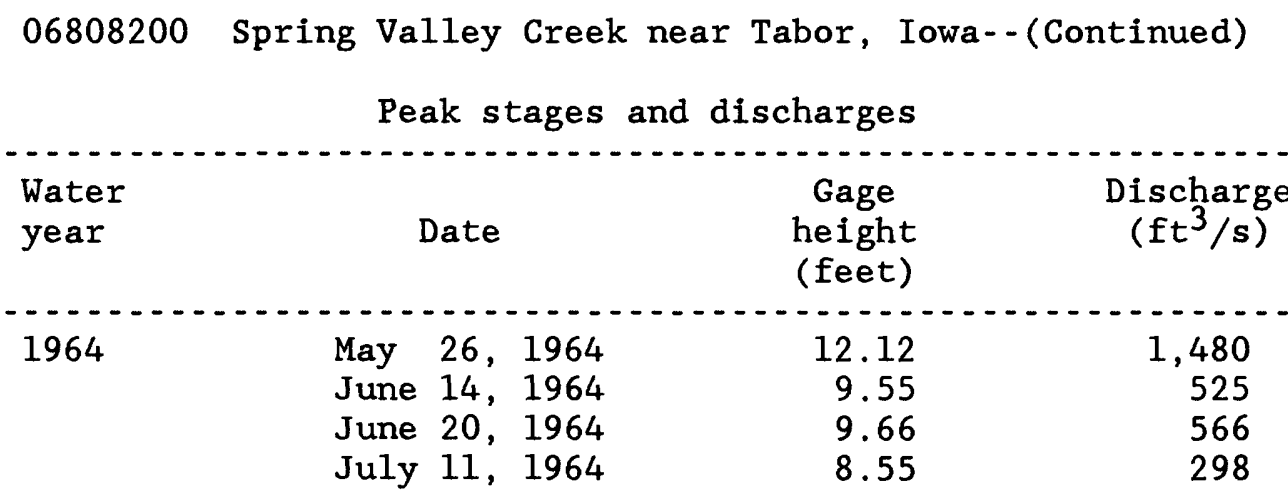

Discontinued September 30, 1964

a Gage height affected by backwater.

b Discharge is an estimate. 
Location. - Lat $40^{\circ} 52^{\prime} 23^{\prime \prime}$, long $95^{\circ} 34^{\prime} 48^{\prime \prime}$, in NE1/4 NE1/4 sec.17, T.70 N., R.41 W., Fremont County, Hydrologic Unit 10240002, on right bank at upstream side of bridge on State Highway 184, $0.3 \mathrm{mi}$ downstream from Deer Creek, $0.5 \mathrm{mi}$ west of Randolph, $16.0 \mathrm{mi}$ upstream from confluence with East Nishnabotna River, and $31.5 \mathrm{mi}$ upstream from mouth of Nishnabotna River.

Drainage area.--1,326 $\mathrm{mi}^{2}$.

Gage.-Water-stage recorder. Datum of gage is $932.99 \mathrm{ft}$ above sea level, unadjusted. Prior to Aug.26, 1955, nonrecording gage with supplementary water-stage recorder operating above $8.4 \mathrm{ft}$ June 30,1949 , to Aug.25, 1955, at same site and datum.

Stage-discharge relation.--Defined by current-meter measurements.

Flood stage.--19 feet.

Remarks.--Base for partial-duration series, 6,500 $\mathrm{ft}^{3} / \mathrm{s}$.

Peak stages and discharges

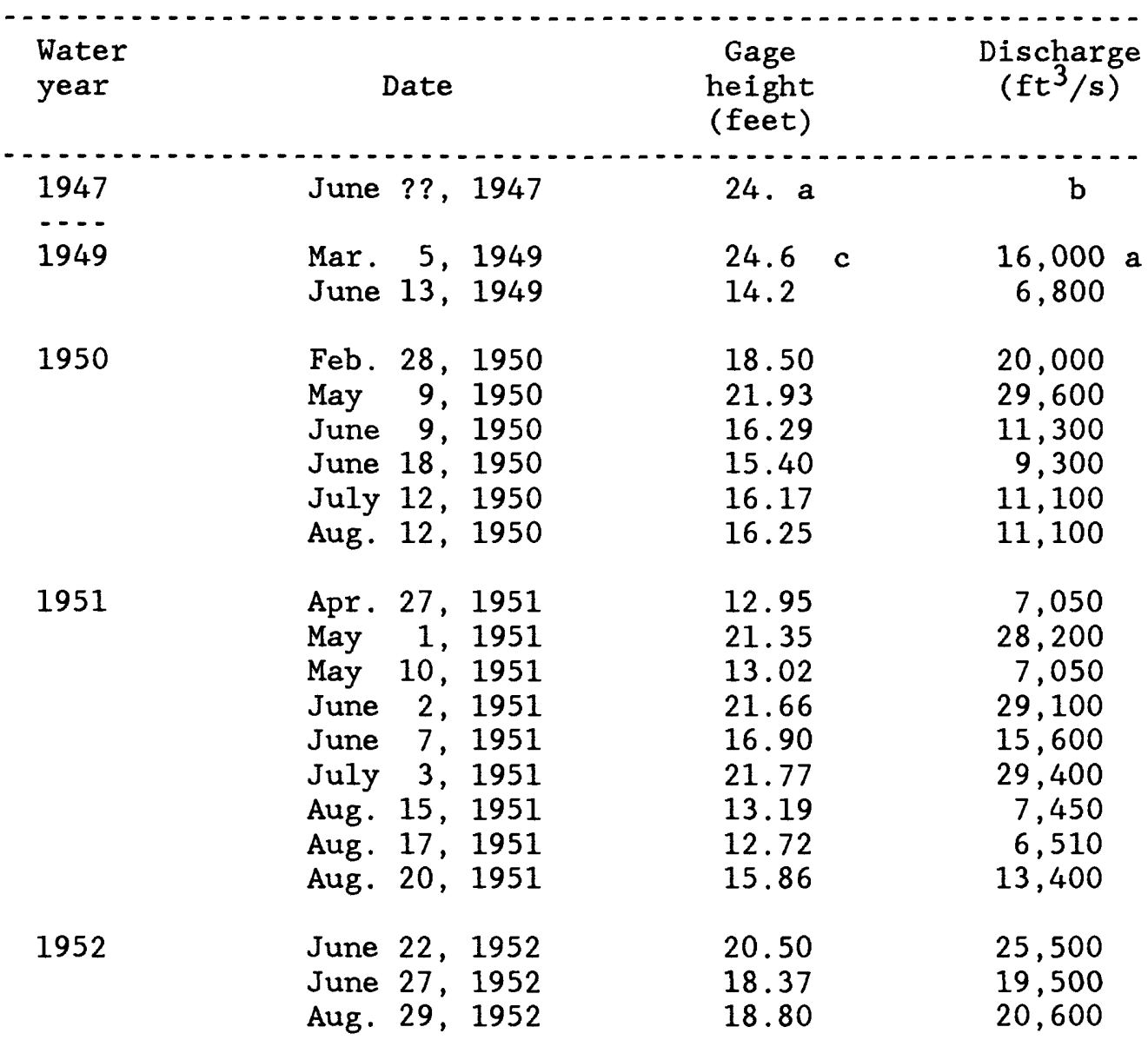


06808500 West Nishnabotna River at Randolph, Iowa--(Continued)

Peak stages and discharges

\begin{tabular}{|c|c|c|c|}
\hline $\begin{array}{l}\text { Water } \\
\text { year }\end{array}$ & Date & $\begin{array}{l}\text { Gage } \\
\text { height } \\
\text { (feet) }\end{array}$ & $\begin{array}{c}\text { Discharge } \\
\left(\mathrm{ft}^{3} / \mathrm{s}\right)\end{array}$ \\
\hline 1953 & June 28,1953 & 11.77 & 4,980 \\
\hline 1954 & Aug. 23, 1954 & 17.15 & 13,400 \\
\hline 1955 & $\begin{array}{cll}\text { Mar. } & 2, & 1955 \\
\text { July } & 9, & 1955\end{array}$ & $\begin{array}{l}14.69 \\
16.18\end{array}$ & $\begin{array}{l}10,500 \\
13,900\end{array}$ \\
\hline 1956 & $\begin{array}{lrl}\text { July } & 8, & 1956 \\
\text { July } & 15, & 1956\end{array}$ & $\begin{array}{l}17.8 \\
14.06\end{array}$ & $\begin{array}{r}17,900 \\
9,260\end{array}$ \\
\hline 1957 & June 16, 1957 & 17.58 & 15,600 \\
\hline 1958 & 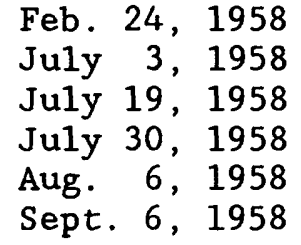 & $\begin{array}{l}18.62 \mathrm{c} \\
17.70 \\
15.35 \\
17.30 \\
16.50 \\
18.20\end{array}$ & $\begin{array}{r}19,100 \text { a } \\
16,500 \\
8,400 \\
14,600 \\
11,800 \\
17,900\end{array}$ \\
\hline 1959 & $\begin{array}{lrr}\text { May } & 3, & 1959 \\
\text { May } & 18, & 1959 \\
\text { May } & 29, & 1959\end{array}$ & $\begin{array}{l}13.15 \\
14.43 \\
18.13\end{array}$ & $\begin{array}{r}6,580 \\
8,200 \\
16,200\end{array}$ \\
\hline 1960 & $\begin{array}{lr}\text { Mar. } 30,1960 \\
\text { May } 6,1960 \\
\text { June } 30,1960 \\
\text { Aug. } 7,1960 \\
\text { Aug. } 29,1960 \\
\text { Sept.18, } 1960\end{array}$ & $\begin{array}{l}19.18 \\
14.42 \\
14.73 \\
14.26 \\
17.16 \\
14.76\end{array}$ & $\begin{array}{r}20,600 \\
7,570 \\
8,200 \\
7,360 \\
14,500 \\
8,410\end{array}$ \\
\hline 1961 & $\begin{array}{ll}\text { Mar. } 13, & 1961 \\
\text { June } 20, & 1961 \\
\text { June } 27, & 1961\end{array}$ & $\begin{array}{l}15.56 \\
14.96 \\
14.36\end{array}$ & $\begin{array}{r}11,700 \\
11,200 \\
7,570\end{array}$ \\
\hline 1962 & $\begin{array}{ll}\text { Mar. } 22, & 1962 \\
\text { May } 21, & 1962 \\
\text { May } 29, & 1962 \\
\text { June } 11, & 1962\end{array}$ & $\begin{array}{l}14.94 \\
14.39 \\
21.60 \\
15.18\end{array}$ & $\begin{array}{r}8,830 \\
7,570 \\
26,600 \\
8,620\end{array}$ \\
\hline 1963 & Mar. 11, 1963 & 15.33 & 12,000 \\
\hline 1964 & $\begin{array}{lll}\text { May } 26, & 1964 \\
\text { June } 14, & 1964 \\
\text { June } 23, & 1964 \\
\text { July } 11, & 1964\end{array}$ & $\begin{array}{l}18.76 \\
16.72 \\
16.30 \\
16.23\end{array}$ & $\begin{array}{l}17,400 \\
11,400 \\
10,400 \\
10,200\end{array}$ \\
\hline
\end{tabular}


06808500 West Nishnabotna River at Randolph, Iowa--(Continued)

Peak stages and discharges

\begin{tabular}{|c|c|c|c|}
\hline $\begin{array}{l}\text { Water } \\
\text { year }\end{array}$ & Date & $\begin{array}{l}\text { Gage } \\
\text { height } \\
\text { (feet) }\end{array}$ & $\begin{array}{l}\text { Discharge } \\
\left(\mathrm{ft}^{3} / \mathrm{s}\right)\end{array}$ \\
\hline 1965 & $\begin{array}{lr}\text { Mar. } 1, & 1965 \\
\text { Mar. } 17, & 1965 \\
\text { Apr. } 1, & 1965 \\
\text { Apr. } & 5,1965 \\
\text { May } 22, & 1965 \\
\text { May } 25, & 1965 \\
\text { June } 29, & 1965 \\
\text { Sept. } 7, & 1965\end{array}$ & $\begin{array}{l}22.76 \mathrm{c} \\
19.25 \\
15.47 \\
15.80 \\
15.37 \\
15.01 \\
18.35 \\
15.33\end{array}$ & $\begin{array}{r}28,000 \text { a } \\
18,400 \\
9,900 \\
11,000 \\
9,670 \\
7,860 \\
17,900 \\
9,580\end{array}$ \\
\hline 1966 & June 26,1966 & 19.38 & 21,200 \\
\hline 1967 & $\begin{array}{l}\text { June } 5,1967 \\
\text { June } 8,1967 \\
\text { June } 10,1967 \\
\text { June } 12,1967 \\
\text { June } 16,1967 \\
\text { June } 21,1967 \\
\text { June } 25,1967\end{array}$ & $\begin{array}{l}19.13 \\
20.54 \\
20.91 \\
17.68 \\
18.52 \\
22.60 \\
14.66\end{array}$ & $\begin{array}{r}20,400 \\
25,600 \\
27,000 \\
15,900 \\
18,500 \\
35,500 \\
8,120\end{array}$ \\
\hline 1968 & June 26,1968 & 9.14 & 944 \\
\hline 1969 & $\begin{array}{lr}\text { Feb. } 26, & 1969 \\
\text { Mar. 18, } & 1969 \\
\text { July 9, } & 1969 \\
\text { July } 18, & 1969\end{array}$ & $\begin{array}{l}15.65 \\
16.67 \\
15.68 \\
14.44\end{array}$ & $\begin{array}{r}10,300 \\
12,900 \\
10,400 \\
7,650\end{array}$ \\
\hline 1970 & May 14,1970 & 13.31 & 5,560 \\
\hline 1971 & $\begin{array}{lll}\text { Feb. } & 19, & 1971 \\
\text { Mar. } & 12, & 1971 \\
\text { May } & 11, & 1971 \\
\text { May } & 18, & 1971\end{array}$ & $\begin{array}{l}19.00 \\
14.90 \\
14.89 \\
14.32\end{array}$ & $\begin{array}{r}20,000 \\
8,620 \\
8,600 \\
7,400\end{array}$ \\
\hline 1972 & $\begin{array}{lr}\text { May } \quad 6,1972 \\
\text { Sept.14, } 1972\end{array}$ & $\begin{array}{l}13.87 \\
21.71\end{array}$ & $\begin{array}{r}6,580 \\
18,500\end{array}$ \\
\hline 1973 & $\begin{array}{lr}\text { Dec. } 29,1972 \\
\text { Jan. } 17,1973 \\
\text { Feb. } 24,1973 \\
\text { Mar. } 1,1973 \\
\text { Mar. } 14,1973 \\
\text { Apr. } 16,1973 \\
\text { Apr. } 30,1973 \\
\text { May } 8,1973 \\
\text { July } 4,1973 \\
\text { Sept. 26, } 1973\end{array}$ & $\begin{array}{l}18.55 \mathrm{c} \\
18.75 \mathrm{c} \\
15.39 \\
14.35 \\
14.30 \\
15.27 \\
14.02 \\
17.72 \\
--. \mathrm{d} \\
19.79\end{array}$ & $\begin{array}{r}12,000 \mathrm{a} \\
13,200 \mathrm{a} \\
9,720 \\
7,460 \\
7,360 \\
9,440 \\
6,830 \\
16,000 \\
10,000 \mathrm{a} \\
19,000\end{array}$ \\
\hline
\end{tabular}


06808500 West Nishnabotna River at Randolph, Iowa--(Continued)

Peak stages and discharges

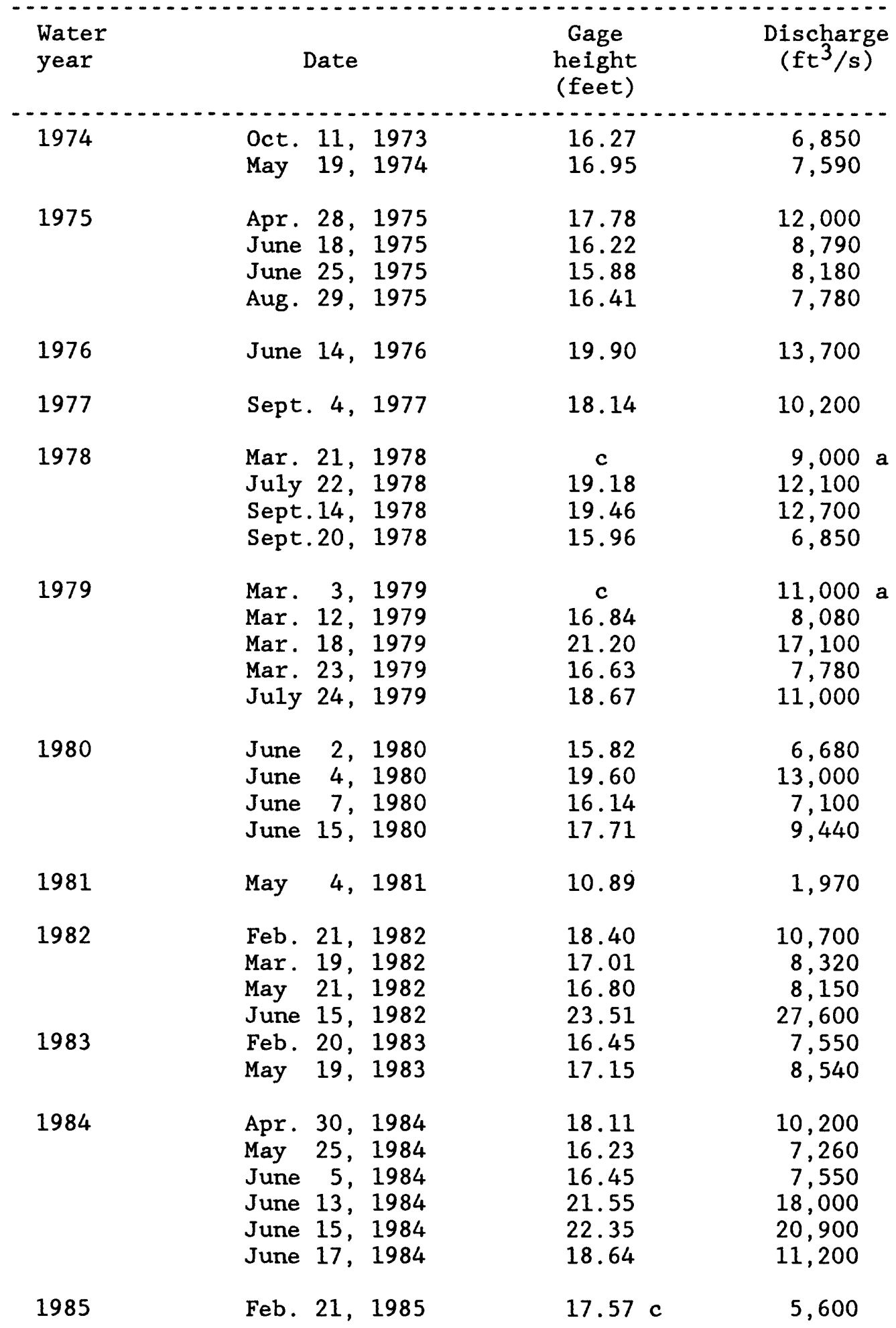


06808500 West Nishnabotna River at Randolph, Iowa--(Continued)

Peak stages and discharges

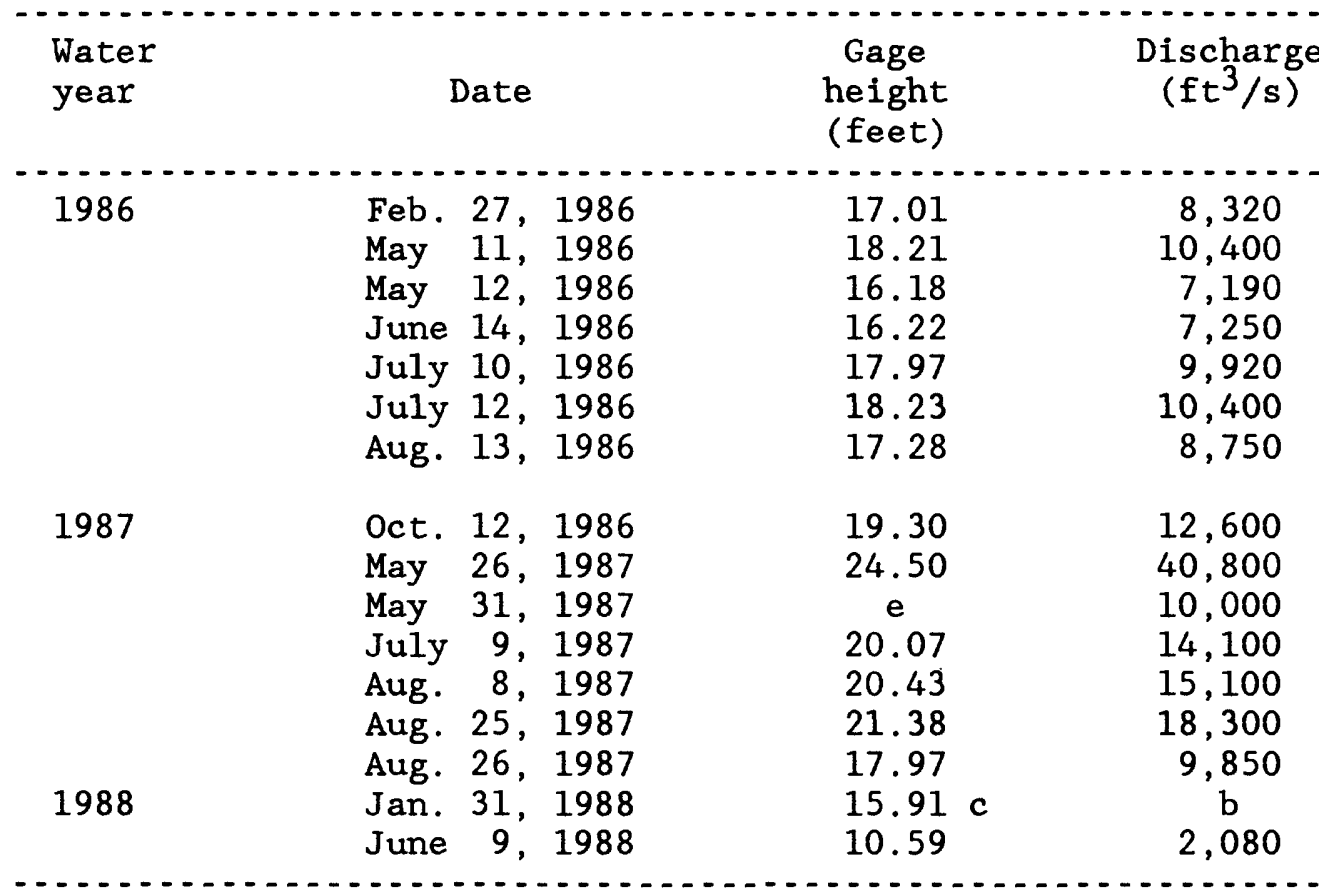

a About. b Discharge not determined. c Affected by ice.

d Gage removed for construction of new bridge.

e Peak occurred during period of surging. 


\section{Bluegrass Creek at Audubon, Iowa}

Location. - - Lat $41^{\circ} 42^{\prime} 46^{\prime \prime}$, long $94^{\circ} 55^{\prime} 43^{\prime \prime}$, in NW1/4 sec. 28, T. 80 N., R.35 W., Audubon County, at bridge on U.S. Highway 71 , near south edge of Audubon.

Drainage area. $--15.4 \mathrm{mi}^{2}$.

Gage.--Crest-stage gage.

Stage-discharge relation.--Undefined due to channel degradation, 1967 discharge determined from an indirect measurement.

Remarks.--Only annual peaks are shown.

Peak stages and discharges

\begin{tabular}{|c|c|c|c|}
\hline $\begin{array}{l}\text { Water } \\
\text { year }\end{array}$ & Date & $\begin{array}{l}\text { Gage } \\
\text { height } \\
\text { (feet) }\end{array}$ & $\begin{array}{c}\text { Discharge } \\
\left(\mathrm{ft}^{3} / \mathrm{s}\right)\end{array}$ \\
\hline 1966 & - & a & b \\
\hline 1967 & June 27,1967 & 85.48 & $2,020 \mathrm{c}$ \\
\hline 1968 & - & a & b \\
\hline 1969 & July 9,1969 & 83.68 & $\mathrm{~b}$ \\
\hline 1970 & May 13,1970 & 81.85 & b \\
\hline 1971 & -- & a & b \\
\hline 1972 & Sept.11, 1972 & 86.05 & b \\
\hline 1973 & July 9,1973 & 84.09 & $\mathrm{~b}$ \\
\hline 1974 & -- & a & b \\
\hline 1975 & Apr. 28, 1975 & 84.62 & b \\
\hline 1976 & -- & a & b \\
\hline 1977 & -- & a & $\mathrm{b}$ \\
\hline 1978 & -- & a & $\mathrm{b}$ \\
\hline 1979 & Mar. 18, 1979 & 84.57 & $\mathrm{~b}$ \\
\hline 1980 & -- & a & $\mathrm{b}$ \\
\hline 1981 & - & $\mathbf{a}$ & b \\
\hline
\end{tabular}


06808880 Bluegrass Creek at Audubon, Iowa--(Continued)

Peak stages and discharges

\begin{tabular}{|c|c|c|c|}
\hline $\begin{array}{l}\text { Water } \\
\text { year }\end{array}$ & Date & $\begin{array}{c}\text { Gage } \\
\text { height } \\
\text { (feet) }\end{array}$ & $\begin{array}{c}\text { Discharge } \\
\left(\mathrm{ft}^{3} / \mathrm{s}\right)\end{array}$ \\
\hline 1982 & Feb. 20,1982 & 83.14 & $\mathrm{~b}$ \\
\hline 1983 & - & a & $\mathrm{b}$ \\
\hline 1984 & - & a & $\mathrm{b}$ \\
\hline 1985 & - & a & $\mathrm{b}$ \\
\hline 1986 & -- & a & $\mathrm{b}$ \\
\hline 1987 & -- & a & $\mathrm{b}$ \\
\hline 1988 & - & a & $\mathrm{b}$ \\
\hline $\begin{array}{ll}\text { a } & \text { Pea } \\
\text { b } & \text { Dis } \\
\text { c } & \text { Dis }\end{array}$ & $\begin{array}{l}\text { did not reach } \\
\text { ot determined. } \\
\text { omputed from ar }\end{array}$ & f gage. & \\
\hline
\end{tabular}




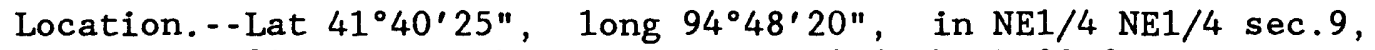
T.79, R. 34 W., Audubon County, on left bank $20 \mathrm{ft}$ downstream from bridge on State Highway 64 (State Highway 44, 1990), 5.2 mi east of Hamlin, and $8 \mathrm{mi}$ upstream from mouth.

Drainage area. $--26.0 \mathrm{mi}^{2}$.

Gage.--Water-stage recorder. Datum of gage is $1,261.54 \mathrm{ft}$ above sea level. Prior to Oct. 1, 1972, at datum $5.00 \mathrm{ft}$ higher.

Stage-discharge relation.--Defined by current-meter measurements below $500 \mathrm{ft}^{3} / \mathrm{s}$ and extended above on basis of slope area measurement of peak flow.

Remarks.--Base for partia1-duration series, $400 \mathrm{ft}^{3} / \mathrm{s}$.

Peak stages and discharges

\begin{tabular}{|c|c|c|c|}
\hline $\begin{array}{l}\text { Water } \\
\text { year }\end{array}$ & Date & $\begin{array}{c}\text { Gage } \\
\text { height } \\
\text { (feet) }\end{array}$ & $\begin{array}{c}\text { Discharge } \\
\left(\mathrm{ft}^{3} / \mathrm{s}\right)\end{array}$ \\
\hline 1952 & $\begin{array}{lr}\text { June } 21, & 1952 \\
\text { June } 27, & 1952 \\
\text { July } 7, & 1952 \\
\text { Aug. 15, } 1952 \\
\text { Aug. 29, } 1952\end{array}$ & $\begin{array}{l}10.72 \\
12.55 \\
10.85 \\
13.07 \\
11.27\end{array}$ & $\begin{array}{l}435 \\
760 \\
450 \\
860 a \\
526\end{array}$ \\
\hline 1953 & $\begin{array}{l}\text { Feb. 19, } 1953 \\
\text { June 4, } 1953 \\
\text { June 10, } 1953\end{array}$ & $\begin{array}{l}10.55 \\
11.18 \\
11.46\end{array}$ & $\begin{array}{l}420 \\
510 \\
558\end{array}$ \\
\hline 1954 & Aug. 23, 1954 & 8.89 & 199 \\
\hline 1955 & Apr. 23, 1955 & 10.27 & 378 \\
\hline 1956 & $\begin{array}{l}\text { June 6, } 1956 \\
\text { Aug. 16, } 1956 \\
\text { Sept. 4, } 1956\end{array}$ & $\begin{array}{l}11.34 \\
11.31 \\
12.41\end{array}$ & $\begin{array}{l}406 \\
406 \\
574\end{array}$ \\
\hline 1957 & June 16, 1957 & 14.80 & 1,160 \\
\hline 1958 & $\begin{array}{lrl}\text { July } & 2, & 1958 \\
\text { July } & 3, & 1958 \\
\text { July } 19, & 1958 \\
\text { Sept. } & 5, & 1958\end{array}$ & $\begin{array}{l}19.35 \\
14.84 \\
12.26 \\
11.08\end{array}$ & $\begin{array}{r}22,700 \\
1,190 \\
625 \\
425\end{array}$ \\
\hline 1959 & May 29,1959 & 9.66 & 240 \\
\hline 1960 & $\begin{array}{rr}\text { Mar. } 29,1960 \\
\text { Aug. } 7,1960\end{array}$ & $\begin{array}{l}12.94 \mathrm{~b} \\
10.70\end{array}$ & $\begin{array}{l}900 \mathrm{c} \\
580\end{array}$ \\
\hline
\end{tabular}


06809000 Davids Creek near Hamlin, Iowa--(Continued)

Peak stages and discharges

\begin{tabular}{|c|c|c|c|}
\hline $\begin{array}{l}\text { Water } \\
\text { year }\end{array}$ & Date & $\begin{array}{l}\text { Gage } \\
\text { height } \\
\text { (feet) }\end{array}$ & $\begin{array}{c}\text { Discharge } \\
\left(\mathrm{ft}^{3} / \mathrm{s}\right)\end{array}$ \\
\hline 1961 & $\begin{array}{l}\text { Feb. } 22,1961 \\
\text { Sept. } 30,1961\end{array}$ & $\begin{array}{l}10.90 \\
10.32\end{array}$ & $\begin{array}{l}620 \\
510\end{array}$ \\
\hline 1962 & $\begin{array}{lr}\text { June } 8, & 1962 \\
\text { June } 19, & 1962\end{array}$ & $\begin{array}{l}13.62 \\
10.43\end{array}$ & $\begin{array}{r}1,550 \\
529\end{array}$ \\
\hline 1963 & May 4,1963 & 8.91 & 322 \\
\hline 1964 & $\begin{array}{lrl}\text { Apr. } & 2, & 1964 \\
\text { Apr. } & 13, & 1964 \\
\text { June } 20, & 1964 \\
\text { June } 22, & 1964\end{array}$ & $\begin{array}{r}10.22 \\
9.82 \\
10.59 \\
13.54\end{array}$ & $\begin{array}{r}795 \\
714 \\
888 \\
2,010\end{array}$ \\
\hline 1965 & $\begin{array}{lr}\text { Mar. } 1, & 1965 \\
\text { Mar. 17, } 1965 \\
\text { Mar. 31, } 1965 \\
\text { Apr. 5, } 1965 \\
\text { June 22, } 1965\end{array}$ & $\begin{array}{r}14.63 \mathrm{~b} \\
14.46 \mathrm{~b} \\
10.30 \\
11.87 \\
9.51\end{array}$ & $\begin{array}{r}1,800 \mathrm{c} \\
1,300 \mathrm{c} \\
928 \\
1,370 \\
723\end{array}$ \\
\hline 1966 & June 12, 1966 & 14.91 & 3,020 \\
\hline 1967 & $\begin{array}{lll}\text { June } 7, & 1967 \\
\text { June } 9, & 1967 \\
\text { June } 12, & 1967\end{array}$ & $\begin{array}{r}9.54 \\
9.24 \\
10.96\end{array}$ & $\begin{array}{r}808 \\
730 \\
1,380\end{array}$ \\
\hline 1968 & $\begin{array}{l}\text { Jan. } 26,1968 \\
\text { Apr. } 23,1968\end{array}$ & $\begin{array}{l}4.18 b \\
3.28\end{array}$ & $\begin{array}{l}d \\
25\end{array}$ \\
\hline 1969 & $\begin{array}{ll}\text { Mar. } 17, & 1969 \\
\text { Mar. } 19,1969 \\
\text { Mar. 24, } 1969 \\
\text { June 28, } 1969\end{array}$ & $\begin{array}{c}12.17 \mathrm{e} \\
6.83 \\
8.19 \\
7.42\end{array}$ & $\begin{array}{r}1,810 \\
487 \\
752 \\
594\end{array}$ \\
\hline 1970 & May 14,1970 & 9.27 & 995 \\
\hline 1971 & $\begin{array}{lrl}\text { Feb. } & 18, & 1971 \\
\text { Mar. } & 13, & 1971 \\
\text { June } & 6, & 1971\end{array}$ & $\begin{array}{l}9.28 \mathrm{~b} \\
7.76 \\
6.48\end{array}$ & $\begin{array}{l}700 \mathrm{c} \\
734 \\
499\end{array}$ \\
\hline 1972 & $\begin{array}{l}\text { Aug. } 1,1972 \\
\text { Sept.11, } 1972\end{array}$ & $\begin{array}{l}5.64 \\
6.04\end{array}$ & $\begin{array}{l}520 \\
584\end{array}$ \\
\hline
\end{tabular}




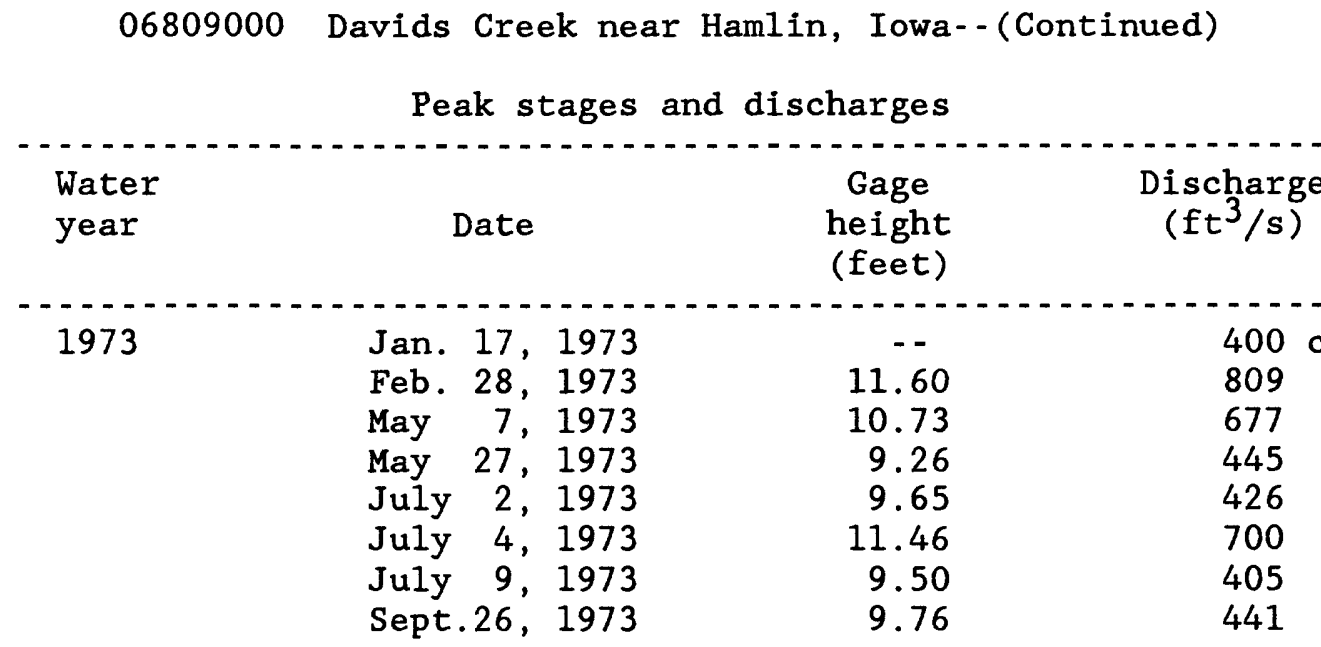

Discontinued Sept. 30, 1973

a Maximum during period June to September 1952.

b Affected by ice. c About.

d Discharge not determined.

e Maximum gage height for water year, $12.85 \mathrm{ft}$, Mar. 17, 1969 (backwater from ice). 
06809210 East Nishnabotna River near Atlantic, Iowa

Location.--Lat $41^{\circ} 20^{\prime} 46^{\prime \prime}$, long $95^{\circ} 04^{\prime} 36^{\prime \prime}$, in NW1/4 NW1/4 sec. 35 , T.76 N., R. 37 W., Cass County, Hydrologic Unit 10240003, on left bank at downstream side of bridge on county road, 1.6 mi upstream from Turkey Creek, $5.2 \mathrm{mi}$ southwest of junction of U.S. Highway 6 and State Highway 83 in Atlantic, $69.1 \mathrm{mi}$ upstream from confluence with West Nishnabotna River, and 84.6 mi upstream from mouth of Nishnabotna River.

Drainage area. $-436 \mathrm{mi}^{2}$.

Gage.--Water-stage encoder. Datum of gage is $1,105.83$ above sea leve1. Prior to 0ct. 1,1970 , at site $2.2 \mathrm{mi}$ upstream at datum $5.00 \mathrm{ft}$ higher.

Stage-discharge relation.--Defined by current-meter measurements.

Flood stage. - - $17 \mathrm{ft}$.

Remarks.--Base for partial-duration series, 3,000 $\mathrm{ft}^{3} / \mathrm{s}$.

Peak stages and discharges

\begin{tabular}{|c|c|c|c|}
\hline $\begin{array}{l}\text { Water } \\
\text { year }\end{array}$ & Date & $\begin{array}{l}\text { Gage } \\
\text { height } \\
\text { (feet) }\end{array}$ & $\begin{array}{c}\text { Discharge } \\
\left(\mathrm{ft}^{3} / \mathrm{s}\right)\end{array}$ \\
\hline 1958 & July 2,1958 & $22.49 \mathrm{a}$ & $34,200 \mathrm{~b}$ \\
\hline 1961 & $\begin{array}{ll}\text { Feb. } 22,1961 \\
\text { Mar. } 14,1961\end{array}$ & $\begin{array}{l}13.08 \\
12.85\end{array}$ & $\begin{array}{l}4,120 \\
3,800\end{array}$ \\
\hline 1962 & $\begin{array}{ll}\text { Mar. } 23,1962 \\
\text { May } 26,1962 \\
\text { May } 29,1962 \\
\text { June } 10,1962\end{array}$ & $\begin{array}{l}12.90 \\
11.30 \\
14.34 \\
12.32\end{array}$ & $\begin{array}{l}6,270 \\
3,970 \\
8,310 \\
4,980\end{array}$ \\
\hline 1963 & $\begin{array}{l}\text { Mar. 4, } 1963 \\
\text { Mar. 11, } 1963\end{array}$ & $\begin{array}{l}13.58 \mathrm{c} \\
11.50\end{array}$ & $\frac{d}{4,090}$ \\
\hline 1964 & 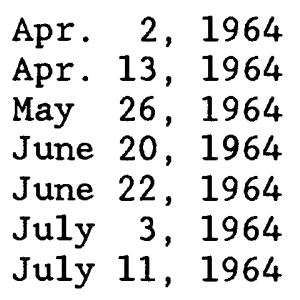 & $\begin{array}{l}13.24 \\
12.61 \\
14.90 \\
17.10 \\
18.16 \\
12.08 \\
13.40\end{array}$ & $\begin{array}{r}6,720 \\
6,270 \\
8,980 \\
13,000 \\
15,200 \\
4,720 \\
6,570\end{array}$ \\
\hline 1965 & 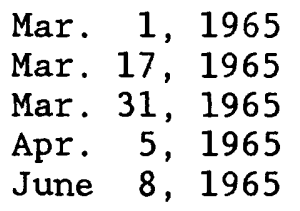 & $\begin{array}{l}20.43 \\
20.10 \\
14.24 \\
16.80 \\
11.54\end{array}$ & $\begin{array}{r}20,500 \\
19,400 \\
7,810 \\
12,300 \\
4,020\end{array}$ \\
\hline
\end{tabular}


06809210 East Nishnabotna River near Atlantic, Iowa--(Continued)

Peak stages and discharges

\begin{tabular}{|c|c|c|c|}
\hline $\begin{array}{l}\text { Water } \\
\text { year }\end{array}$ & Date & $\begin{array}{c}\text { Gage } \\
\text { height } \\
\text { (feet) }\end{array}$ & $\begin{array}{c}\text { Discharge } \\
\left(\mathrm{ft}^{3} / \mathrm{s}\right)\end{array}$ \\
\hline 1966 & June 12, 1966 & $17.86 \mathrm{a}$ & 14,500 \\
\hline 1967 & $\begin{array}{l}\text { June } 8,1967 \\
\text { June 10, } 1967 \\
\text { June 12, } 1967 \\
\text { June 16, } 1967 \\
\text { June 24, } 1967 \\
\text { June 28, } 1967\end{array}$ & $\begin{array}{l}13.10 \\
12.00 \\
14.37 \\
13.87 \\
11.70 \\
13.64\end{array}$ & $\begin{array}{l}5,490 \\
4,380 \\
8,100 \\
7,220 \\
4,020 \\
6,940\end{array}$ \\
\hline 1968 & June 30,1968 & 7.20 & 560 \\
\hline 1969 & $\begin{array}{lr}\text { Feb. } 27, & 1969 \\
\text { Mar. } 17, & 1969 \\
\text { Mar. } 20, & 1969 \\
\text { Mar. } 24, & 1969 \\
\text { June } 28, & 1969 \\
\text { July } 9, & 1969\end{array}$ & $\begin{array}{l}14.85 \mathrm{c} \\
17.65 \\
11.37 \\
12.61 \\
12.95 \\
15.46\end{array}$ & $\begin{array}{c}7,000 \text { e } \\
14,100 \\
4,040 \\
5,930 \\
6,030 \\
9,980\end{array}$ \\
\hline 1970 & $\begin{array}{lrl}\text { Mar. } & 2, & 1970 \\
\text { May } & 11, & 1970 \\
\text { May } 14, & 1970\end{array}$ & $\begin{array}{l}16.00 \\
14.00 \\
13.90\end{array}$ & $\begin{array}{r}11,000 \\
7,510 \\
7,300\end{array}$ \\
\hline 1971 & $\begin{array}{lr}\text { Feb. } 19,1971 \\
\text { Mar. } 13,1971 \\
\text { June } 6,1971\end{array}$ & $\begin{array}{r}17.35 \\
10.48 \\
9.77\end{array}$ & $\begin{array}{r}15,200 \\
4,320 \\
3,590\end{array}$ \\
\hline 1972 & $\begin{array}{l}\text { Aug. } 2,1972 \\
\text { Sept.12, } 1972\end{array}$ & $\begin{array}{r}9.42 \\
22.81\end{array}$ & $\begin{array}{r}3,240 \\
26,700\end{array}$ \\
\hline 1973 & $\begin{array}{lr}\text { Dec. } 30,1972 \\
\text { Jan. } 17,1973 \\
\text { Feb. 1, } 1973 \\
\text { Feb. } 24,1973 \\
\text { Mar. 1, } 1973 \\
\text { Mar. 11, } 1973 \\
\text { Mar. 31, } 1973 \\
\text { Apr. 15, } 1973 \\
\text { May 7, } 1973 \\
\text { May 28, } 1973 \\
\text { Ju1y 1, } 1973 \\
\text { Ju1y 4, } 1973 \\
\text { Sept.26, } 1973\end{array}$ & $\begin{array}{r}-- \\
12.10 \\
9.43 \\
9.25 \\
9.21 \\
9.15 \\
10.67 \\
10.31 \\
14.63 \\
10.20 \\
10.50 \\
12.21 \\
9.61\end{array}$ & $\begin{array}{c}3,800 \text { e } \\
6,340 \\
3,250 \\
3,070 \\
3,740 \\
3,680 \\
4,540 \\
4,290 \\
10,400 \\
4,160 \\
4,440 \\
6,490 \\
3,610\end{array}$ \\
\hline 1974 & 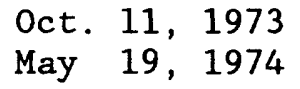 & $\begin{array}{r}11.65 \\
9.30\end{array}$ & $\begin{array}{l}5,720 \\
3,270\end{array}$ \\
\hline
\end{tabular}


06809210 East Nishnabotna River near Atlantic, Iowa--(Continued)

Peak stages and discharges

\begin{tabular}{|c|c|c|c|}
\hline $\begin{array}{l}\text { Water } \\
\text { year }\end{array}$ & Date & $\begin{array}{l}\text { Gage } \\
\text { height } \\
\text { (feet) }\end{array}$ & $\begin{array}{c}\text { Discharge } \\
\left(\mathrm{ft}^{3} / \mathrm{s}\right)\end{array}$ \\
\hline 1975 & $\begin{array}{ll}\text { Mar. } 21, & 1975 \\
\text { Apr. } 28, & 1975 \\
\text { June } 18, & 1975 \\
\text { June } 25, & 1975\end{array}$ & $\begin{array}{r}11.07 \\
12.20 \\
9.85 \\
11.78\end{array}$ & $\begin{array}{l}5,720 \\
6,480 \\
3,790 \\
5,890\end{array}$ \\
\hline 1976 & $\begin{array}{l}\text { Mar. } 12,1976 \\
\text { June } 14,1976\end{array}$ & $\begin{array}{r}9.06 \\
12.34\end{array}$ & $\begin{array}{l}3,050 \\
6,680\end{array}$ \\
\hline 1977 & Sept. 3, 1977 & 10.29 & 4,230 \\
\hline 1978 & $\begin{array}{l}\text { Mar. } 20,1978 \\
\text { Sept.13, } 1978\end{array}$ & $\begin{array}{l}12.25 \\
12.00\end{array}$ & $\begin{array}{l}6,440 \\
6,280\end{array}$ \\
\hline 1979 & $\begin{array}{l}\text { Mar. } 13,1979 \\
\text { Mar. } 18,1979\end{array}$ & $\begin{array}{l}10.41 \\
18.42\end{array}$ & $\begin{array}{r}4,680 \\
18,500\end{array}$ \\
\hline 1980 & $\begin{array}{l}\text { June } 6,1980 \\
\text { June } 15,1980\end{array}$ & $\begin{array}{l}14.25 \\
16.50\end{array}$ & $\begin{array}{r}9,500 \\
13,500\end{array}$ \\
\hline 1981 & June 15, 1981 & 7.21 & 1,650 \\
\hline 1982 & $\begin{array}{lll}\text { Feb. } & 20,1982 \\
\text { Mar. } & 19,1982 \\
\text { May } & 20,1982 \\
\text { May } & 26,1982 \\
\text { Aug. } & 30,1982\end{array}$ & $\begin{array}{r}12.40 \\
11.99 \\
9.15 \\
10.99 \\
8.75\end{array}$ & $\begin{array}{r}6,730 \\
10,300 \\
5,470 \\
8,620 \\
4,860\end{array}$ \\
\hline 1983 & 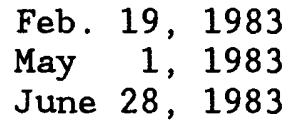 & $\begin{array}{l}9.64 \\
9.20 \\
9.13\end{array}$ & $\begin{array}{l}3,580 \\
3,290 \\
3,160\end{array}$ \\
\hline 1984 & $\begin{array}{ll}\text { Apr. } 27, & 1984 \\
\text { Apr. } & 30,1984 \\
\text { May } 25, & 1984 \\
\text { June } 16, & 1984\end{array}$ & $\begin{array}{r}9.87 \\
15.42 \\
9.79 \\
10.95\end{array}$ & $\begin{array}{r}4,250 \\
12,600 \\
4,170 \\
5,430\end{array}$ \\
\hline 1985 & Feb. 21,1985 & 10.93 & 5,400 \\
\hline
\end{tabular}


06809210 East Nishnabotna River near Atlantic, Iowa--(Continued)

Peak stages and discharges

\begin{tabular}{|c|c|c|c|}
\hline $\begin{array}{l}\text { Water } \\
\text { year }\end{array}$ & Date & $\begin{array}{c}\text { Gage } \\
\text { height } \\
\text { (feet) }\end{array}$ & $\begin{array}{c}\text { Discharge } \\
\left(\mathrm{ft}^{3} / \mathrm{s}\right)\end{array}$ \\
\hline 1986 & $\begin{array}{ll}\text { Feb. } 26,1986 \\
\text { May } 10,1986 \\
\text { May 11, } 1986 \\
\text { May 15, } 1986 \\
\text { May 16, } 1986 \\
\text { June 14, } 1986 \\
\text { June } 30,1986 \\
\text { July 9, } 1986 \\
\text { July 10, } 1986 \\
\text { July } 14,1986 \\
\text { Aug. 13, } 1986 \\
\text { Sept.19, } 1986 \\
\text { Sept.29, } 1986\end{array}$ & $\begin{array}{r}12.76 \\
12.81 \\
11.68 \\
8.74 \\
10.62 \\
8.72 \\
9.94 \\
8.65 \\
9.26 \\
11.63 \\
12.55 \\
9.63 \\
8.70\end{array}$ & $\begin{array}{l}7,850 \\
7,920 \\
6,310 \\
3,160 \\
5,050 \\
3,150 \\
4,320 \\
3,090 \\
3,640 \\
6,240 \\
7,530 \\
4,010 \\
3,130\end{array}$ \\
\hline 1987 & $\begin{array}{ll}\text { Oct. } 11, & 1986 \\
\text { July } 12, & 1987 \\
\text { Aug. } 26, & 1987\end{array}$ & $\begin{array}{r}13.55 \\
10.05 \\
9.59\end{array}$ & $\begin{array}{l}9,090 \\
4,440 \\
3,970\end{array}$ \\
\hline 1988 & $\begin{array}{lrl}\text { Jan. } & 30, & 1988 \\
\text { June } & 8, & 1988\end{array}$ & $\begin{array}{l}9.73 c \\
6.94\end{array}$ & $\begin{array}{c}d \\
2,040\end{array}$ \\
\hline
\end{tabular}

a From floodmark.

b Discharge computed from an indirect measurement.

c Affected by ice.

d Discharge not determined. e About. 


\section{East Nishnabotna River at Red Oak, Iowa}

Location. - - Lat $41^{\circ} 00^{\prime} 31^{\prime \prime}$, long $95^{\circ} 14^{\prime} 29^{\prime \prime}$, in NW1/4 SE1/4 sec. 29 , T.72 N., R. 38 W., Montgomery County, Hydrologic Unit 10240003, on upstream side of Coolbaugh Street and $200 \mathrm{ft}$ left of left end of Coolbaugh Street bridge in Red Oak, $0.2 \mathrm{mi}$ upstream from Red Oak Creek, $38.0 \mathrm{mi}$ upstream from confluence with West Nishnabotna River, and $53.6 \mathrm{mi}$ upstream from mouth of Nishnabotna River.

Drainage area. $--894 \mathrm{mi}^{2}$.

Gage.--Water-stage recorder. Datum of gage is $1,005.45 \mathrm{ft}$ above sea level. Prior to July 5, 1925, nonrecording gage at present site at datum $4.60 \mathrm{ft}$ higher. May 29, 1936, to Nov. 13, 1952, nonrecording gage with supplementary water-stage recorder in operation above $3.2 \mathrm{ft}$ gage height July 30, 1939 to Nov. 13, 1952 , and Nov. 14, 1952, to June 13, 1966, water-stage recorder, all at site $0.5 \mathrm{mi}$ upstream at datum $5.00 \mathrm{ft}$ higher. June 14, 1966, to Sept. 30, 1969, at present site at datum $5.00 \mathrm{ft}$ higher.

Stage-discharge relation.--Defined by current-meter measurements.

Flood stage. - -18 ft.

Remarks.--Base for partial-duration series, 4,500 $\mathrm{ft}^{3} / \mathrm{s}$.

Peak stages and discharges

\begin{tabular}{|c|c|c|c|}
\hline $\begin{array}{l}\text { Water } \\
\text { year }\end{array}$ & Date & $\begin{array}{c}\text { Gage } \\
\text { height } \\
\text { (feet) }\end{array}$ & $\begin{array}{c}\text { Discharge } \\
\left(\mathrm{ft}^{3} / \mathrm{s}\right)\end{array}$ \\
\hline 1917 & June 7,1917 & $21.7 \mathrm{a}$ & 23,500 \\
\hline 1918 & May 29,1918 & 13.6 & 3,740 \\
\hline 1919 & June 13, 1919 & 13.1 & 3,300 \\
\hline 1920 & Apr. 21,1920 & 12.6 & 2,920 \\
\hline 1921 & Sept.21, 1921 & 12.4 & 2,800 \\
\hline 1922 & Sept. 2, 1922 & 13.6 & 3,740 \\
\hline 1923 & Sept.29, 1923 & 12.2 & 2,700 \\
\hline 1924 & $\begin{array}{l}\text { June } 9,1924 \\
\text { June } 26,1924\end{array}$ & $\begin{array}{l}16.5 \\
18.2\end{array}$ & $\begin{array}{r}6,800 \\
10,000\end{array}$ \\
\hline 1925 & June 25,1925 & 10.00 & 1,850 \\
\hline 1936 & Sept.16, 1936 & 14.8 & 3,800 \\
\hline
\end{tabular}


06809500 East Nishnabotna River at Red Oak, Iowa--(Continued)

Peak stages and discharges

\begin{tabular}{|c|c|c|c|}
\hline $\begin{array}{l}\text { Water } \\
\text { year }\end{array}$ & Date & $\begin{array}{l}\text { Gage } \\
\text { height } \\
\text { (feet) }\end{array}$ & $\begin{array}{c}\text { Discharge } \\
\left(\mathrm{ft}^{3} / \mathrm{s}\right)\end{array}$ \\
\hline 1937 & $\begin{array}{l}\text { Feb. } 19,1937 \\
\text { Mar. } 4,1937\end{array}$ & $\begin{array}{l}15.7 \\
18.6\end{array}$ & $\begin{array}{l}4,800 \\
9,600\end{array}$ \\
\hline 1938 & Sept. 14, 1938 & 14.9 & 3,810 \\
\hline 1939 & Aug. 12, 1939 & 18.2 & 9,070 \\
\hline 1940 & Aug. 13, 1940 & 15.83 & 5,000 \\
\hline 1941 & $\begin{array}{l}\text { June } 4,1941 \\
\text { June } 10,1941\end{array}$ & $\begin{array}{l}15.5 \\
15.4\end{array}$ & $\begin{array}{l}4,580 \\
4,550\end{array}$ \\
\hline 1942 & $\begin{array}{lll}\text { May } & 12 & 1942 \\
\text { June } & 29, & 1942 \\
\text { July } & 20, & 1942\end{array}$ & $\begin{array}{l}15.9 \\
16.1 \\
18.4\end{array}$ & $\begin{array}{l}5,100 \\
5,300 \\
8,100\end{array}$ \\
\hline 1943 & $\begin{array}{l}\text { Feb. } 4,1943 \\
\text { May } \quad 16,1943\end{array}$ & $\begin{array}{l}17.9 \\
16.8\end{array}$ & $\begin{array}{l}8,610 \\
6,810\end{array}$ \\
\hline 1944 & $\begin{array}{lrr}\text { May } & 22, & 1944 \\
\text { June } & 14, & 1944 \\
\text { Aug. } & 2, & 1944\end{array}$ & $\begin{array}{l}17.7 \\
16.0 \\
17.9\end{array}$ & $\begin{array}{l}8,200 \\
5,900 \\
8,500\end{array}$ \\
\hline 1945 & $\begin{array}{ll}\text { Mar. } 11, & 1945 \\
\text { Apr. } 24, & 1945 \\
\text { May } 15, & 1945 \\
\text { May } 23, & 1945 \\
\text { May } 28, & 1945 \\
\text { June } 1, & 1945 \\
\text { June } 7, & 1945 \\
\text { June } 16, & 1945\end{array}$ & $\begin{array}{l}15.5 \\
16.5 \\
17.6 \\
20.5 \\
15.8 \\
17.0 \\
17.2 \\
14.5\end{array}$ & $\begin{array}{r}5,400 \\
6,400 \\
8,000 \\
16,100 \\
8,000 \\
9,800 \\
10,100 \\
6,300\end{array}$ \\
\hline 1946 & $\begin{array}{lr}\text { Jan. } & 6,1946 \\
\text { Feb. } & 6,1946 \\
\text { Mar. } & 6,1946 \\
\text { Mar. } 13, & 1946 \\
\text { May } & 3,1946 \\
\text { June } 18, & 1946 \\
\text { Aug. } 26, & 1946\end{array}$ & $\begin{array}{l}16.4 \mathrm{~b} \\
18.2 \\
13.5 \\
12.9 \\
12.6 \\
14.3 \\
18.2\end{array}$ & $\begin{array}{r}6,400 \mathrm{c} \\
12,000 \\
5,350 \\
4,850 \\
4,600 \\
6,090 \\
12,000\end{array}$ \\
\hline
\end{tabular}


06809500 East Nishnabotna River at Red Oak, Iowa--(Continued)

Peak stages and discharges

\begin{tabular}{|c|c|c|c|c|}
\hline $\begin{array}{l}\text { Water } \\
\text { year }\end{array}$ & & Date & $\begin{array}{l}\text { Gage } \\
\text { height } \\
\text { (feet) }\end{array}$ & $\begin{array}{c}\text { Discharge } \\
\left(\mathrm{ft}^{3} / \mathrm{s}\right)\end{array}$ \\
\hline 1947 & $\begin{array}{l}\text { Oct. } \\
\text { Apr. } \\
\text { May } \\
\text { June } \\
\text { June } \\
\text { June } \\
\text { June } \\
\text { June }\end{array}$ & $\begin{array}{r}18,1946 \\
11,1947 \\
15,1947 \\
2,1947 \\
5,1947 \\
13,1947 \\
18,1947 \\
23,1947\end{array}$ & $\begin{array}{l}14.3 \\
16.1 \\
12.5 \\
19.0 \\
18.7 \\
23.2 \\
12.1 \\
19.6\end{array}$ & $\begin{array}{r}6,800 \\
8,900 \\
5,150 \\
13,900 \\
13,400 \\
36,200 \\
7,100 \\
15,800\end{array}$ \\
\hline 1948 & $\begin{array}{l}\text { Feb. } \\
\text { Mar. } \\
\text { July } \\
\text { July }\end{array}$ & $\begin{array}{ll}28, & 1948 \\
19, & 1948 \\
21, & 1948 \\
28, & 1948\end{array}$ & $\begin{array}{l}15.8 \\
18.9 \\
12.0 \\
10.3\end{array}$ & $\begin{array}{r}12,400 \\
18,400 \\
7,000 \\
5,200\end{array}$ \\
\hline 1949 & $\begin{array}{l}\text { Mar. } \\
\text { Mar. } \\
\text { June } \\
\text { July }\end{array}$ & $\begin{array}{rr}5, & 1949 \\
22, & 1949 \\
2, & 1949 \\
20, & 1949\end{array}$ & $\begin{array}{r}17.7 \\
9.8 \\
9.6 \\
9.8\end{array}$ & $\begin{array}{r}15,800 \\
4,720 \\
4,540 \\
4,720\end{array}$ \\
\hline 1950 & $\begin{array}{l}\text { Feb. } \\
\text { Feb. } \\
\text { May } \\
\text { June }\end{array}$ & $\begin{array}{rr}9, & 1950 \\
28, & 1950 \\
9, & 1950 \\
18, & 1950\end{array}$ & $\begin{array}{l}10.5 \\
11.8 \\
11.2 \\
11.4\end{array}$ & $\begin{array}{l}6,550 \\
8,580 \\
7,620 \\
7,940\end{array}$ \\
\hline 1951 & $\begin{array}{l}\text { Mar. } \\
\text { Apr. } \\
\text { May } \\
\text { June } \\
\text { June } \\
\text { July } \\
\text { Aug. }\end{array}$ & $\begin{array}{rr}29, & 1951 \\
25, & 1951 \\
1, & 1951 \\
2, & 1951 \\
8, & 1951 \\
3, & 1951 \\
20, & 1951\end{array}$ & $\begin{array}{r}14.31 \\
9.55 \\
16.97 \\
14.1 \\
13.7 \\
14.9 \\
10.9\end{array}$ & $\begin{array}{r}10,700 \\
4,850 \\
15,200 \\
10,400 \\
9,920 \\
11,600 \\
6,190\end{array}$ \\
\hline 1952 & $\begin{array}{l}\text { Mar. } \\
\text { Mar. } \\
\text { June } \\
\text { June } \\
\text { July } \\
\text { July } \\
\text { Aug. } \\
\text { Aug. }\end{array}$ & 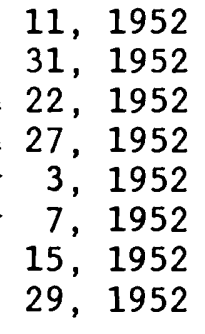 & $\begin{array}{r}10.56 \\
11.58 \\
15.53 \\
13.54 \\
9.95 \\
11.28 \\
10.22 \\
12.58\end{array}$ & $\begin{array}{r}5,860 \\
7,020 \\
12,600 \\
9,500 \\
5,250 \\
6,660 \\
5,450 \\
8,280\end{array}$ \\
\hline 1953 & June & 10,1953 & 7.77 & 3,250 \\
\hline 1954 & Aug. & 23,1954 & 14.08 & 10,400 \\
\hline
\end{tabular}


06809500 East Nishnabotna River at Red Oak, Iowa--(Continued)

Peak stages and discharges

\begin{tabular}{|c|c|c|c|}
\hline $\begin{array}{l}\text { Water } \\
\text { year }\end{array}$ & Date & $\begin{array}{l}\text { Gage } \\
\text { height } \\
\text { (feet) }\end{array}$ & $\begin{array}{c}\text { Discharge } \\
\left(\mathrm{ft}^{3} / \mathrm{s}\right)\end{array}$ \\
\hline 1955 & $\begin{array}{l}\text { Mar. 3, } 1955 \\
\text { Apr. 24, } 1955\end{array}$ & $\begin{array}{l}13.58 \\
10.19\end{array}$ & $\begin{array}{l}9,640 \\
5,450\end{array}$ \\
\hline 1956 & Sept. 5, 1956 & 12.57 & 7,630 \\
\hline 1957 & $\begin{array}{lll}\text { May } & 30, & 1957 \\
\text { June } & 17, & 1957\end{array}$ & $\begin{array}{l}11.35 \\
15.12\end{array}$ & $\begin{array}{r}6,780 \\
11,800\end{array}$ \\
\hline 1958 & 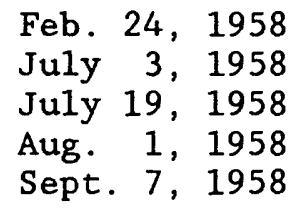 & $\begin{array}{l}12.17 \mathrm{~b} \\
22.27 \\
13.20 \\
10.30 \\
18.58\end{array}$ & $\begin{array}{r}7,710 \mathrm{c} \\
35,600 \\
9,700 \\
5,960 \\
19,900\end{array}$ \\
\hline 1959 & 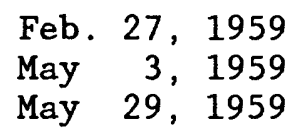 & $\begin{array}{l}9.25 \mathrm{~b} \\
10.35 \\
15.14\end{array}$ & $\begin{array}{r}4,800 \mathrm{c} \\
6,080 \\
12,700\end{array}$ \\
\hline 1960 & 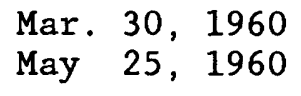 & $\begin{array}{r}16.40 \\
9.25\end{array}$ & $\begin{array}{r}15,100 \\
5,000\end{array}$ \\
\hline 1961 & $\begin{array}{ll}\text { Feb. } 23, & 1961 \\
\text { Mar. } 15, & 1961\end{array}$ & $\begin{array}{l}10.88 \\
12.25\end{array}$ & $\begin{array}{l}6,880 \\
8,510\end{array}$ \\
\hline 1962 & 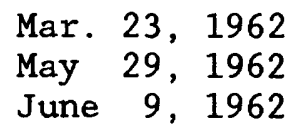 & $\begin{array}{l}12.00 \\
15.28 \\
11.61\end{array}$ & $\begin{array}{r}9,460 \\
13,000 \\
7,730\end{array}$ \\
\hline 1963 & Mar. 11,1963 & 10.90 & 8,920 \\
\hline 1964 & $\begin{array}{lrl}\text { Apr. } & 2, & 1964 \\
\text { Apr. } & 13, & 1964 \\
\text { May } 26, & 1964 \\
\text { June } 20, & 1964 \\
\text { June } 23, & 1964 \\
\text { July } 3, & 1964 \\
\text { July } 11, & 1964\end{array}$ & $\begin{array}{r}10.87 \\
10.38 \\
14.43 \\
15.00 \\
15.96 \\
9.33 \\
12.77\end{array}$ & $\begin{array}{r}10,700 \\
9,700 \\
11,600 \\
13,200 \\
14,300 \\
5,000 \\
9,320\end{array}$ \\
\hline 1965 & $\begin{array}{lr}\text { Mar. } 2, & 1965 \\
\text { Mar. 17, } 1965 \\
\text { Apr. 1, } 1965 \\
\text { Apr. } 5,1965 \\
\text { June 29, } 1965\end{array}$ & $\begin{array}{l}19.40 \\
17.01 \\
11.94 \\
14.96 \\
11.42\end{array}$ & $\begin{array}{r}22,200 \\
16,300 \\
8,170 \\
12,400 \\
7,500\end{array}$ \\
\hline
\end{tabular}


06809500 East Nishnabotna River at Red Oak, Iowa--(Continued)

Peak stages and discharges

\begin{tabular}{|c|c|c|c|}
\hline $\begin{array}{l}\text { Water } \\
\text { year }\end{array}$ & Date & $\begin{array}{l}\text { Gage } \\
\text { height } \\
\text { (feet) }\end{array}$ & $\begin{array}{l}\text { Discharge } \\
\left(\mathrm{ft}^{3} / \mathrm{s}\right)\end{array}$ \\
\hline 1966 & $\begin{array}{ll}\text { June } 12,1966 \\
\text { June } 26,1966\end{array}$ & $\begin{array}{r}15.56 \\
9.24\end{array}$ & $\begin{array}{r}15,100 \\
-1 .- \\
6,040\end{array}$ \\
\hline 1967 & 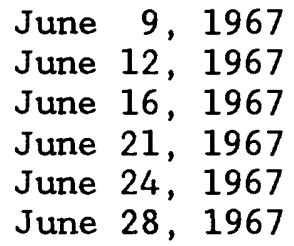 & $\begin{array}{r}14.02 \\
13.71 \\
14.05 \\
11.35 \\
9.30 \\
8.90\end{array}$ & $\begin{array}{r}12,300 \\
11,900 \\
12,400 \\
8,600 \\
6,140 \\
5,700\end{array}$ \\
\hline 1968 & May 16,1968 & 1.29 & 355 \\
\hline 1969 & $\begin{array}{lr}\text { Feb. } 27,1969 \\
\text { Mar. } 18,1969 \\
\text { Mar. 20, } 1969 \\
\text { Mar. } 25,1969 \\
\text { June } 29,1969 \\
\text { July } 9,1969\end{array}$ & $\begin{array}{r}11.22 \\
14.48 \\
7.75 \\
8.65 \\
10.40 \\
12.25 \\
--.--\end{array}$ & $\begin{array}{r}8,830 \\
13,700 \\
4,890 \\
5,810 \\
6,730 \\
9,020\end{array}$ \\
\hline 1970 & $\begin{array}{lrl}\text { Mar. } & 2, & 1970 \\
\text { May } & 12, & 1970 \\
\text { May } & 14, & 1970\end{array}$ & $\begin{array}{l}18.59 \\
15.84 \\
17.59\end{array}$ & $\begin{array}{r}11,700 \\
8,790 \\
11,400\end{array}$ \\
\hline 1971 & $\begin{array}{lrl}\text { Feb. } & 19, & 1971 \\
\text { Mar. } & 14, & 1971 \\
\text { June } & 6, & 1971\end{array}$ & $\begin{array}{l}20.76 \\
13.94 \\
13.92\end{array}$ & $\begin{array}{r}17,000 \\
6,830 \\
6,810\end{array}$ \\
\hline 1972 & Sept.13, 1972 & 27.43 & 38,000 \\
\hline 1973 & $\begin{array}{lr}\text { Dec. } 30,1972 \\
\text { Jan. } 17,1973 \\
\text { Feb. } 2,1973 \\
\text { Mar. 1, } 1973 \\
\text { Mar. 14, } 1973 \\
\text { Mar. } 31,1973 \\
\text { Apr. 16, } 1973 \\
\text { May } 8,1973 \\
\text { May 28, } 1973 \\
\text { July 4, } 1973 \\
\text { Sept.26, } 1973\end{array}$ & $\begin{array}{l}15.47 \\
17.73 \\
14.35 \\
12.72 \\
13.25 \\
14.50 \\
14.37 \\
19.20 \\
13.40 \\
15.49 \\
13.08\end{array}$ & $\begin{array}{r}7,810 \\
10,900 \\
6,500 \\
4,810 \\
5,310 \\
6,660 \\
6,520 \\
14,500 \\
6,240 \\
8,740 \\
5,460\end{array}$ \\
\hline 1974 & $\begin{array}{lr}\text { Oct. } 11,1973 \\
\text { Mar. } 3,1974\end{array}$ & $\begin{array}{l}15.28 \\
12.08\end{array}$ & $\begin{array}{l}8,460 \\
4,600\end{array}$ \\
\hline
\end{tabular}


06809500 East Nishnabotna River at Red Oak, Iowa--(Continued)

Peak stages and discharges

\begin{tabular}{|c|c|c|c|}
\hline $\begin{array}{l}\text { Water } \\
\text { year }\end{array}$ & Date & $\begin{array}{l}\text { Gage } \\
\text { height } \\
\text { (feet) }\end{array}$ & $\begin{array}{c}\text { Discharge } \\
\left(\mathrm{ft}^{3} / \mathrm{s}\right)\end{array}$ \\
\hline 1975 & $\begin{array}{l}\text { Mar. 21, } 1975 \\
\text { Mar. 28, } 1975 \\
\text { Apr. 28, } 1975 \\
\text { June 19, } 1975 \\
\text { June 25, } 1975\end{array}$ & $\begin{array}{l}15.00 \\
12.40 \\
15.84 \\
12.72 \\
14.19\end{array}$ & $\begin{array}{l}7,600 \\
4,730 \\
8,610 \\
5,050 \\
6,630\end{array}$ \\
\hline 1976 & June 14, 1976 & 18.78 & 12,800 \\
\hline 1977 & $\begin{array}{l}\text { Aug. 28, } 1977 \\
\text { Aug. 31, } 1977 \\
\text { Sept. 2, } 1977\end{array}$ & $\begin{array}{l}14.35 \\
13.55 \\
13.33\end{array}$ & $\begin{array}{l}6,820 \\
5,900 \\
5,660\end{array}$ \\
\hline 1978 & $\begin{array}{l}\text { Mar. } 21,1978 \\
\text { Apr. } 18,1978 \\
\text { Sept.14, } 1978\end{array}$ & $\begin{array}{l}16.34 \\
13.82 \\
15.70\end{array}$ & $\begin{array}{l}9,280 \\
6,270 \\
8,440\end{array}$ \\
\hline 1979 & $\begin{array}{l}\text { Mar. 13, } 1979 \\
\text { Mar. 19, } 1979 \\
\text { Mar. 23, } 1979 \\
\text { Mar. 29, } 1979 \\
\text { June 27, } 1979\end{array}$ & $\begin{array}{l}15.34 \\
21.20 \\
12.78 \\
12.17 \\
14.83\end{array}$ & $\begin{array}{r}8,010 \\
16,900 \\
5,110 \\
4,500 \\
7,400\end{array}$ \\
\hline 1980 & $\begin{array}{l}\text { June 4, } 1980 \\
\text { June 6, } 1980 \\
\text { June 15, } 1980\end{array}$ & $\begin{array}{l}13.43 \\
18.44 \\
19.60\end{array}$ & $\begin{array}{r}5,750 \\
12,300 \\
14,200\end{array}$ \\
\hline 1981 & June 16,1981 & 8.48 & 1,710 \\
\hline 1982 & $\begin{array}{ll}\text { Feb. } 20, & 1982 \\
\text { Feb. } 21, & 1982 \\
\text { Mar. } 19,1982 \\
\text { May 26, } 1982 \\
\text { June 15, } 1982\end{array}$ & $\begin{array}{l}19.94 \mathrm{~b} \\
19.22 \\
16.50 \\
14.74 \\
18.86\end{array}$ & $\begin{array}{r}\mathrm{d} \\
13,600 \\
9,530 \\
7,290 \\
13,000\end{array}$ \\
\hline 1983 & $\begin{array}{lr}\text { Dec. } & 28,1982 \\
\text { Feb. } & 20,1983 \\
\text { May } & 2,1983\end{array}$ & $\begin{array}{l}12.24 \\
13.71 \\
12.91\end{array}$ & $\begin{array}{l}4,510 \\
6,060 \\
5,190\end{array}$ \\
\hline 1984 & $\begin{array}{lr}\text { Apr. } 27,1984 \\
\text { Apr. } 30,1984 \\
\text { May } 25,1984 \\
\text { June } 8,1984 \\
\text { June } 13,1984 \\
\text { June } 15,1984 \\
\text { June } 16,1984\end{array}$ & $\begin{array}{l}15.06 \\
20.11 \\
14.63 \\
13.08 \\
13.70 \\
18.28 \\
16.61\end{array}$ & $\begin{array}{r}7,680 \\
15,000 \\
7,150 \\
5,370 \\
6,050 \\
12,100 \\
9,680\end{array}$ \\
\hline
\end{tabular}


06809500 East Nishnabotna River at Red Oak, Iowa--(Continued)

Peak stages and discharges

\begin{tabular}{|c|c|c|c|}
\hline $\begin{array}{l}\text { Water } \\
\text { year }\end{array}$ & Date & $\begin{array}{c}\text { Gage } \\
\text { height } \\
\text { (feet) }\end{array}$ & $\begin{array}{c}\text { Discharge } \\
\left(\mathrm{ft}^{3} / \mathrm{s}\right)\end{array}$ \\
\hline 1985 & Feb. 21, 1985 & 14.55 & 7,890 \\
\hline 1986 & $\begin{array}{lr}\text { Feb. } 27,1986 \\
\text { Apr. } & 4,1986 \\
\text { May } 10,1986 \\
\text { May } 17,1986 \\
\text { June } 30,1986 \\
\text { July } 10,1986 \\
\text { July } 14,1986 \\
\text { Aug. } 13,1986 \\
\text { Sept.19, } 1986\end{array}$ & $\begin{array}{c}15.71 \\
12.65 \\
17.36 \\
-- \\
-- \\
12.92 \\
13.15 \\
-- \\
12.60\end{array}$ & $\begin{array}{r}8,050 \\
4,770 \\
10,200 \\
6,700 \\
4,800 \\
5,030 \\
5,260 \\
7,200 \\
4,730\end{array}$ \\
\hline 1987 & $\begin{array}{lr}\text { Oct. } 12,1986 \\
\text { May } 26,1987 \\
\text { July 9, } 1987 \\
\text { July } 12,1987 \\
\text { Aug. } 8,1987 \\
\text { Aug. } 26,1987\end{array}$ & $\begin{array}{l}17.08 \\
14.32 \\
14.74 \\
13.39 \\
14.15\end{array}$ & $\begin{array}{l}d \\
9,820 \\
6,460 \\
6,930 \\
5,490 \\
6,280\end{array}$ \\
\hline 1988 & $\begin{array}{lrl}\text { Jan. } & 30, & 1988 \\
\text { June } & 9, & 1988\end{array}$ & $\begin{array}{c}13.90 \mathrm{~b} \\
7.84\end{array}$ & $\underset{1,170}{d}$ \\
\hline
\end{tabular}


Location. - Lat $40^{\circ} 37^{\prime} 57^{\prime \prime}$, long $95^{\circ} 37^{\prime} 32^{\prime \prime}$, in SW1/4 SEl/4 sec. 11 , T.67 N., R.42 W., Fremont County, Hydrologic Unit 10240004, on left bank $1.7 \mathrm{mi}$ downstream from confluence of East Nishnabotna and West Nishnabotna Rivers, $2 \mathrm{mi}$ northeast of Hamburg, and $13.8 \mathrm{mi}$ upstream from mouth.

Drainage area.--2,806 $\mathrm{mi}^{2}$.

Gage.--Water-stage encoder. Datum of gage is $894.17 \mathrm{ft}$ above sea level. Prior to Oct.1, 1923, chain gage at site 6 mi downstream at different datum. Oct.5, 1928, to Sept.6, 1929, chain gage at site $1,000 \mathrm{ft}$ upstream at datum $0.42 \mathrm{ft}$ higher. Sept. 7, 1929, to Feb.11, 1935, chain gage and Feb.12, 1935, to June 5, 1947, wire-weight gage, at present site and datum. June 6 to July 22, 1947, staff gage at site 1,000 ft upstream at different datum. July 23, 1947, to Nov.16, 1950, staff gage at present site and datum.

Stage-discharge relation.--Defined by current-meter measurements.

Flood stage.--16 feet.

Remarks.--Base for partial-duration series, 9,000 $\mathrm{ft}^{3} / \mathrm{s}$.

Peak stages and discharges

\begin{tabular}{|c|c|c|c|}
\hline $\begin{array}{l}\text { Water } \\
\text { year }\end{array}$ & Date & $\begin{array}{c}\text { Gage } \\
\text { height } \\
\text { (feet) }\end{array}$ & $\begin{array}{c}\text { Discharge } \\
\left(\mathrm{ft}^{3} / \mathrm{s}\right)\end{array}$ \\
\hline 1917 & June 7, 1917 & 21. $\mathrm{a} \mathrm{b}$ & c \\
\hline 1922 & Apr. 12, 1922 & 16.7 & 11,800 \\
\hline 1923 & Mar. 27, 1923 & 16.00 & 8,800 \\
\hline 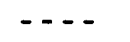 & - & $-\ldots$ & - \\
\hline 1929 & $\begin{array}{lr}\text { Mar. } 13, & 1929 \\
\text { July } & 6,1929 \\
\text { July } 15, & 1929\end{array}$ & $\begin{array}{l}22.3 \\
18.2 \\
19.10\end{array}$ & $\begin{array}{r}21,100 \\
9,500 \\
11,100\end{array}$ \\
\hline 1930 & Feb. 8,1930 & $13.8 \mathrm{~d}$ & $3,020 a$ \\
\hline 1931 & June 22, 1931 & 18.1 & 9,350 \\
\hline 1932 & $\begin{array}{l}\text { Nov. } 25,1931 \\
\text { Aug. } 16,1932\end{array}$ & $\begin{array}{l}19.1 \\
19.1\end{array}$ & $\begin{array}{l}11,100 \\
10,300\end{array}$ \\
\hline 1933 & Jan. 22,1933 & 17.1 & 8,020 \\
\hline 1934 & June 9,1934 & 14.6 & 5,620 \\
\hline
\end{tabular}


06810000 Nishnabotna River above Hamburg, Iowa--(Continued)

Peak stages and discharges

\begin{tabular}{|c|c|c|c|}
\hline $\begin{array}{l}\text { Water } \\
\text { year }\end{array}$ & Date & $\begin{array}{l}\text { Gage } \\
\text { height } \\
\text { (feet) }\end{array}$ & $\begin{array}{c}\text { Discharge } \\
\left(\mathrm{ft}^{3} / \mathrm{s}\right)\end{array}$ \\
\hline 1935 & June 26,1935 & 17.5 & 8,500 \\
\hline 1936 & Mar. 5, 1936 & 22.2 & 20,600 \\
\hline 1937 & Mar. 5, 1937 & 21.6 & 16,500 \\
\hline 1938 & $\begin{array}{l}\text { Aug. } 28,1938 \\
\text { Sept.14, } 1938\end{array}$ & $\begin{array}{l}17.9 \\
18.2\end{array}$ & $\begin{array}{l}9,060 \\
9,650\end{array}$ \\
\hline 1939 & $\begin{array}{l}\text { Mar. 12, } 1939 \\
\text { Aug. } 11,1939\end{array}$ & $\begin{array}{l}23.0 \mathrm{~d} \\
17.9\end{array}$ & $\begin{array}{r}22,100 a \\
9,060\end{array}$ \\
\hline 1940 & $\begin{array}{lr}\text { Aug. } & 8,1940 \\
\text { Aug. } 12,1940 \\
\text { Aug. } 14,1940 \\
\text { Aug. } 17,1940\end{array}$ & $\begin{array}{l}18.4 \\
18.9 \\
18.2 \\
18.4\end{array}$ & $\begin{array}{r}9,800 \\
10,700 \\
9,500 \\
9,800\end{array}$ \\
\hline 1941 & $\begin{array}{ll}\text { June } 9, & 1941 \\
\text { Sept. } 15, & 1941\end{array}$ & $\begin{array}{l}20.4 \\
19.1\end{array}$ & $\begin{array}{l}14,600 \\
11,100\end{array}$ \\
\hline 1942 & $\begin{array}{ll}\text { Oct. } 22, & 1941 \\
\text { June } 20, & 1942 \\
\text { June } 25, & 1942\end{array}$ & $\begin{array}{l}18.8 \\
19.2 \\
19.2\end{array}$ & $\begin{array}{l}10,500 \\
11,700 \\
11,400\end{array}$ \\
\hline 1943 & $\begin{array}{lrl}\text { May } & 16, & 1943 \\
\text { June } & 3, & 1943 \\
\text { June } & 5, & 1943 \\
\text { Aug. } & 3, & 1943\end{array}$ & $\begin{array}{l}19.1 \\
18.6 \\
18.6 \\
18.9\end{array}$ & $\begin{array}{l}11,100 \\
10,100 \\
10,100 \\
10,700\end{array}$ \\
\hline 1944 & $\begin{array}{lr}\text { May } & 26,1944 \\
\text { June } & 4,1944 \\
\text { June } 9, & 1944 \\
\text { June } 15, & 1944\end{array}$ & $\begin{array}{l}17.9 \\
19.5 \\
18.8 \\
20.8\end{array}$ & $\begin{array}{r}9,060 \\
11,600 \\
9,200 \\
11,800\end{array}$ \\
\hline 1945 & $\begin{array}{lr}\text { Mar. } 11, & 1945 \\
\text { Apr. } 23, & 1945 \\
\text { May } 15, & 1945 \\
\text { May } 23, & 1945 \\
\text { June } 3, & 1945\end{array}$ & $\begin{array}{l}18.6 \mathrm{~d} \\
18.8 \\
18.4 \\
22.6 \\
18.0\end{array}$ & $\begin{array}{r}9,350 \text { a } \\
10,500 \\
9,800 \\
21,000 \\
9,200\end{array}$ \\
\hline 1946 & $\begin{array}{l}\text { Feb. } 6,1946 \\
\text { June } 18,1946 \\
\text { Sept. } 4,1946\end{array}$ & $\begin{array}{l}18.9 \\
19.1 \\
19.7\end{array}$ & $\begin{array}{l}10,700 \\
11,100 \\
11,300\end{array}$ \\
\hline
\end{tabular}


06810000 Nishnabotna River above Hamburg, Iowa--(Continued)

Peak stages and discharges

\begin{tabular}{|c|c|c|c|}
\hline $\begin{array}{l}\text { Water } \\
\text { year }\end{array}$ & Date & $\begin{array}{l}\text { Gage } \\
\text { height } \\
\text { (feet) }\end{array}$ & $\begin{array}{c}\text { Discharge } \\
\left(\mathrm{ft}^{3} / \mathrm{s}\right)\end{array}$ \\
\hline \multirow[t]{2}{*}{1947} & $\begin{array}{cr}\text { Apr. } 10,1947 \\
\text { June } & 2,1947\end{array}$ & $\begin{array}{l}19.5 \\
19.80\end{array}$ & $\begin{array}{r}9,800 \\
11,200\end{array}$ \\
\hline & $\begin{array}{l}\text { June } 6,1947 \\
\text { June } 14,1947 \\
\text { June } 24,1947\end{array}$ & $\begin{array}{l}23.0 \\
25.7 \\
26.03 \mathrm{~b}\end{array}$ & $\begin{array}{l}28,700 \\
52,100 \\
55,500\end{array}$ \\
\hline 1948 & $\begin{array}{ll}\text { Feb. } 28, & 1948 \\
\text { Mar. } 20, & 1948\end{array}$ & $\begin{array}{l}21.5 \\
24.5\end{array}$ & $\begin{array}{l}17,600 \\
36,300\end{array}$ \\
\hline 1949 & $\begin{array}{ll}\text { Mar. } & 7,1949 \\
\text { June } 2, & 1949 \\
\text { June } 28, & 1949\end{array}$ & $\begin{array}{l}25.9 \\
19.2 \\
18.5\end{array}$ & $\begin{array}{r}32,200 \\
12,000 \\
9,950\end{array}$ \\
\hline 1950 & 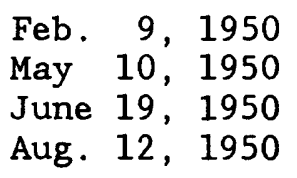 & $\begin{array}{l}18.4 \\
22.5 \\
18.4 \\
19.2\end{array}$ & $\begin{array}{l}10,500 \\
19,900 \\
10,500 \\
12,000\end{array}$ \\
\hline 1951 & $\begin{array}{lrl}\text { Mar. } & 29, & 1951 \\
\text { May } & 2, & 1951 \\
\text { June } & 3, & 1951 \\
\text { June } & 8, & 1951 \\
\text { June } & 20, & 1951 \\
\text { July } & 4, & 1951\end{array}$ & $\begin{array}{l}21.46 \\
24.51 \\
24.32 \\
21.0 \\
17.9 \\
21.89\end{array}$ & $\begin{array}{r}16,200 \\
23,800 \\
23,200 \\
14,500 \\
9,170 \\
16,600\end{array}$ \\
\hline 1952 & $\begin{array}{ll}\text { Mar. } 13, & 1952 \\
\text { June } 23, & 1952 \\
\text { June } 28, & 1952 \\
\text { Aug. } 29, & 1952\end{array}$ & $\begin{array}{l}18.2 \\
20.92 \\
20.6 \\
20.20\end{array}$ & $\begin{array}{l}10,700 \\
12,800 \\
12,300 \\
11,800\end{array}$ \\
\hline 1953 & June 9,1953 & 14.65 & 5,440 \\
\hline 1954 & Aug. 23, 1954 & 21.32 & 12,600 \\
\hline 1955 & $\begin{array}{lrr}\text { Apr. } & 24, & 1955 \\
\text { July } & 9, & 1955\end{array}$ & $\begin{array}{l}18.8 \\
18.64\end{array}$ & $\begin{array}{l}12,000 \\
10,600\end{array}$ \\
\hline 1956 & $\begin{array}{lll}\text { July } & 8, & 1956 \\
\text { Sept. } & 6, & 1956\end{array}$ & $\begin{array}{l}20.90 \\
18.38\end{array}$ & $\begin{array}{r}13,500 \\
9,760\end{array}$ \\
\hline 1957 & $\begin{array}{l}\text { June } 7,1957 \\
\text { June } 16,1957\end{array}$ & $\begin{array}{l}18.90 \\
21.59\end{array}$ & $\begin{array}{l}13,400 \\
20,400\end{array}$ \\
\hline
\end{tabular}


06810000 Nishnabotna River above Hamburg, Iowa--(Continued)

Peak stages and discharges

\begin{tabular}{|c|c|c|c|}
\hline $\begin{array}{l}\text { Water } \\
\text { year }\end{array}$ & Date & $\begin{array}{l}\text { Gage } \\
\text { height } \\
\text { (feet) }\end{array}$ & $\begin{array}{c}\text { Discharge } \\
\left(\mathrm{ft}^{3} / \mathrm{s}\right)\end{array}$ \\
\hline 1958 & $\begin{array}{lrr}\text { July } & 6, & 1958 \\
\text { July } 19, & 1958 \\
\text { July } 30, & 1958 \\
\text { Aug. } & 6, & 1958 \\
\text { Sept. } & 6, & 1958\end{array}$ & $\begin{array}{l}22.75 \\
19.45 \\
20.43 \\
20.53 \\
20.55\end{array}$ & $\begin{array}{l}14,600 \\
10,000 \\
11,300 \\
11,400 \\
10,300\end{array}$ \\
\hline 1959 & $\begin{array}{lll}\text { May } & 19, & 1959 \\
\text { May } & 29, & 1959\end{array}$ & $\begin{array}{l}17.90 \\
21.70\end{array}$ & $\begin{array}{r}9,640 \\
15,400\end{array}$ \\
\hline 1960 & Mar. 31, 1960 & 24.56 & 23,900 \\
\hline 1961 & $\begin{array}{ll}\text { Feb. } 23,1961 \\
\text { Mar. } 13,1961 \\
\text { Mar. } 15,1961\end{array}$ & $\begin{array}{l}18.30 \\
21.33 \\
20.35\end{array}$ & $\begin{array}{r}9,240 \\
14,400 \\
12,600\end{array}$ \\
\hline 1962 & $\begin{array}{lrl}\text { Mar. } & 23, & 1962 \\
\text { May } & 29, & 1962 \\
\text { June } & 9, & 1962\end{array}$ & $\begin{array}{l}21.60 \\
23.62 \\
19.05\end{array}$ & $\begin{array}{r}16,200 \\
18,000 \\
9,500\end{array}$ \\
\hline 1963 & Mar. 12, 1963 & 22.02 & 15,600 \\
\hline 1964 & $\begin{array}{lll}\text { May } & 26, & 1964 \\
\text { June } & 20, & 1964 \\
\text { June } 23, & 1964 \\
\text { July } 11, & 1964\end{array}$ & $\begin{array}{l}23.32 \\
22.02 \\
22.72 \\
21.80\end{array}$ & $\begin{array}{l}19,900 \\
16,200 \\
17,300 \\
15,400\end{array}$ \\
\hline 1965 & 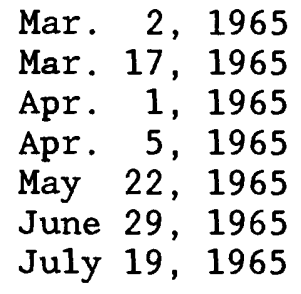 & $\begin{array}{l}24.83 \\
24.20 \\
18.90 \\
21.45 \\
20.69 \\
22.30 \\
20.05\end{array}$ & $\begin{array}{l}24,700 \\
22,600 \\
10,100 \\
14,800 \\
13,200 \\
17,000 \\
11,300\end{array}$ \\
\hline 1966 & $\begin{array}{ll}\text { June } 13, & 1966 \\
\text { June } 26, & 1966\end{array}$ & $\begin{array}{c}18.70 \\
--\end{array}$ & $\begin{array}{r}9,330 \\
16,000 \text { a }\end{array}$ \\
\hline 1967 & $\begin{array}{ll}\text { June } 10, & 1967 \\
\text { June } 12, & 1967 \\
\text { June } 16,1967 \\
\text { June } 21,1967\end{array}$ & $\begin{array}{l}24.53 \mathrm{e} \\
24.47 \mathrm{e} \\
23.40 \mathrm{e} \\
24.17 \mathrm{e}\end{array}$ & $\begin{array}{l}20,000 \mathrm{a} \\
19,000 \mathrm{a} \\
12,500 \mathrm{a} \\
12,000 \mathrm{a}\end{array}$ \\
\hline 1968 & June 27,1968 & 8.85 & 798 \\
\hline
\end{tabular}


06810000 Nishnabotna River above Hamburg, Iowa--(Continued)

Peak stages and discharges

\begin{tabular}{|c|c|c|c|}
\hline $\begin{array}{l}\text { Water } \\
\text { year }\end{array}$ & Date & $\begin{array}{c}\text { Gage } \\
\text { height } \\
\text { (feet) }\end{array}$ & $\begin{array}{c}\text { Discharge } \\
\left(\mathrm{ft}^{3} / \mathrm{s}\right)\end{array}$ \\
\hline 1969 & $\begin{array}{ll}\text { Mar. } 18, & 1969 \\
\text { Ju1y } 10, & 1969 \\
\text { July } 18, & 1969\end{array}$ & $\begin{array}{l}24.19 \\
21.07 \\
23.23\end{array}$ & $\begin{array}{l}19,700 \\
12,300 \\
17,100\end{array}$ \\
\hline 1970 & 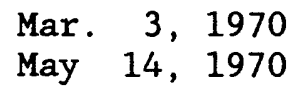 & $\begin{array}{l}20.05 \\
19.12\end{array}$ & $\begin{array}{l}10,300 \\
10,400\end{array}$ \\
\hline 1971 & 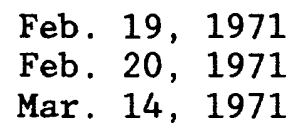 & $\begin{array}{c}26.25 \mathrm{~d} \\
-- \\
18.64\end{array}$ & $\begin{array}{c}c \\
25,000 \text { a } \\
9,700\end{array}$ \\
\hline 1972 & Sept. 15, 1972 & 27.42 & 25,200 \\
\hline 1973 & $\begin{array}{lr}\text { Dec. } 30,1972 \\
\text { Jan. } 18,1973 \\
\text { Feb. } 1,1973 \\
\text { Feb. } 24,1973 \\
\text { Mar. } 15,1973 \\
\text { Apr. 1, } 1973 \\
\text { Apr. 17, } 1973 \\
\text { May } 8,1973 \\
\text { May } 28,1973 \\
\text { Ju1y } 4,1973 \\
\text { Sept.27, } 1973\end{array}$ & $\begin{array}{l}22.19 \\
24.3 \mathrm{~d} \\
19.80 \\
19.72 \\
18.98 \\
20.62 \\
20.75 \\
23.38 \\
19.92 \\
24.39 \\
23.45\end{array}$ & $\begin{array}{r}16,700 \\
16,000 \text { a } \\
11,500 \\
10,000 \\
9,250 \\
11,400 \\
11,600 \\
16,900 \\
10,300 \\
17,300 \\
17,900\end{array}$ \\
\hline 1974 & Oct. 12,1973 & 23.07 & 16,300 \\
\hline 1975 & $\begin{array}{ll}\text { Mar. } 21, & 1975 \\
\text { Apr. } 28, & 1975 \\
\text { June } 25, & 1975\end{array}$ & $\begin{array}{l}18.99 \\
21.63 \\
20.75\end{array}$ & $\begin{array}{l}12,600 \\
13,300 \\
11,600\end{array}$ \\
\hline 1976 & June 14, 1976 & 25.70 & 21,400 \\
\hline 1977 & $\begin{array}{l}\text { Aug. } 31,1977 \\
\text { Sept. 4, } 1977\end{array}$ & $\begin{array}{l}23.16 \\
23.24\end{array}$ & $\begin{array}{l}12,000 \\
12,200\end{array}$ \\
\hline 1978 & $\begin{array}{ll}\text { Mar. } 21, & 1978 \\
\text { Apr. } 17, & 1978 \\
\text { July } 22, & 1978 \\
\text { Sept. } 15, & 1978\end{array}$ & $\begin{array}{l}24.98 \\
22.98 \\
24.72 \\
25.17\end{array}$ & $\begin{array}{l}17,700 \\
15,000 \\
19,300 \\
20,500\end{array}$ \\
\hline 1979 & $\begin{array}{lr}\text { Mar } & 3,1979 \\
\text { Mar. } & 7,1979 \\
\text { Mar. } 13, & 1979 \\
\text { Mar. } & 19,1979 \\
\text { July } 24, & 1979\end{array}$ & $\begin{array}{c}-- \\
27.46 \mathrm{~d} \\
22.43 \\
-- \\
22.43\end{array}$ & $\begin{array}{c}16,000 \\
c \\
13,900 \\
23,000 \\
13,900\end{array}$ \\
\hline
\end{tabular}


06810000 Nishnabotna River above Hamburg, Iowa--(Continued)

Peak stages and discharges

\begin{tabular}{|c|c|c|c|}
\hline $\begin{array}{l}\text { Water } \\
\text { year }\end{array}$ & Date & $\begin{array}{c}\text { Gage } \\
\text { height } \\
\text { (feet) }\end{array}$ & $\begin{array}{c}\text { Discharge } \\
\left(\mathrm{ft}^{3} / \mathrm{s}\right)\end{array}$ \\
\hline 1980 & $\begin{array}{lll}\text { June } & 4,1980 \\
\text { June } 7, & 1980 \\
\text { June } 15, & 1980\end{array}$ & $\begin{array}{l}24.93 \\
-- \\
23.20 \mathrm{~b}\end{array}$ & $\begin{array}{l}19,800 \\
15,000 \\
15,500\end{array}$ \\
\hline 1981 & July 9,1981 & 13.29 & 3,190 \\
\hline 1982 & $\begin{array}{ll}\text { Feb. } 22, & 1982 \\
\text { Apr. } 16,1982 \\
\text { May } 21,1982 \\
\text { June } 15,1982 \\
\text { Aug. } 15,1982\end{array}$ & $\begin{array}{l}23.17 \\
20.63 \\
21.37 \\
27.25 \\
20.73\end{array}$ & $\begin{array}{l}17,000 \\
10,800 \\
11,800 \\
29,100 \\
10,700\end{array}$ \\
\hline 1983 & $\begin{array}{ll}\text { Feb. } 15,1983 \\
\text { Feb. } 20,1983 \\
\text { June } 18,1983 \\
\text { June } 30,1983\end{array}$ & $\begin{array}{l}18.98 \\
20.88 \\
19.78 \\
20.81\end{array}$ & $\begin{array}{r}9,350 \\
12,700 \\
9,040 \\
10,800\end{array}$ \\
\hline 1984 & $\begin{array}{lr}\text { Mar. } 26,1984 \\
\text { Apr. } 27,1984 \\
\text { Apr. } 30,1984 \\
\text { June } 5,1984 \\
\text { June } 8,1984 \\
\text { June } 9,1984 \\
\text { June } 13,1984 \\
\text { June } 16,1984\end{array}$ & $\begin{array}{l}22.29 \\
21.13 \\
25.65 \\
20.96 \\
20.46 \\
23.71 \\
27.08 \\
27.90\end{array}$ & $\begin{array}{l}13,600 \\
11,400 \\
21,800 \\
11,000 \\
10,200 \\
16,700 \\
21,900 \\
24,300\end{array}$ \\
\hline 1985 & Feb. 22, 1985 & $21.90 \mathrm{~d}$ & 12,300 \\
\hline 1986 & $\begin{array}{lr}\text { Feb. } 27, & 1986 \\
\text { May } 11, & 1986 \\
\text { May } 17, & 1986 \\
\text { June } 15, & 1986 \\
\text { July } 6, & 1986 \\
\text { July } 10, & 1986 \\
\text { July } 12, & 1986 \\
\text { July } 14, & 1986 \\
\text { Aug. } 14, & 1986 \\
\text { Sept. 20, } & 1986\end{array}$ & $\begin{array}{c}23.87 \\
23.87 \\
21.71 \\
19.76 \\
19.82 \\
23.18 \\
-- \\
-- \\
19.90 \\
21.08\end{array}$ & $\begin{array}{r}18,300 \\
18,300 \\
13,000 \\
9,150 \\
9,260 \\
16,500 \\
c \\
c \\
9,400 \\
11,600\end{array}$ \\
\hline 1987 & $\begin{array}{lr}\text { Oct. } 12, & 1986 \\
\text { May } 27, & 1987 \\
\text { July } 9, & 1987 \\
\text { July } 13, & 1987 \\
\text { Aug. } 8, & 1987 \\
\text { Aug. } 27, & 1987 \\
\text { Sept.16, } & 1987\end{array}$ & $\begin{array}{l}25.95 \\
28.14 \\
23.78 \\
21.30 \\
22.88 \\
25.35 \\
22.42\end{array}$ & $\begin{array}{l}24,500 \\
31,400 \\
18,000 \\
12,100 \\
15,700 \\
22,600 \\
14,600\end{array}$ \\
\hline
\end{tabular}


06810000 Nishnabotna River above Hamburg, Iowa--(Continued)

Peak stages and discharges

Water
year

a About.

b From floodmark, present site and datum.

c Discharge not determined.

d Affected by ice.

e Backwater from Missouri River. 
Table 5. Temporary bench marks, Nishnabotna River basin, Iowa

The majority of the temporary bench marks listed in this tabulation are those for which elevations have been established by the Water Resources Division, Iowa District, U.S. Geological Survey. For several bridges, bench-mark elevations were obtained from the Iowa Department of Transportation or from county engineers. The work was done as part of a stream profile project cooperatively financed by the Highway Research Advisory Board, Highway Division, Iowa Department of Transportation and the U.S. Geological Survey.

Stream reaches surveyed in the Nishnabotna River basin for the establishment of bench-mark elevations include the west Nishnabotna River from near Manning, and the East Nishnabotna River from near Hamlin, to the Nishnabotna River at Hamburg; and Indian creek from near Emerson to the mouth. Bench marks were set at most bridges and at intermediate points between bridges, generally at road intersections at 1 mile intervals.

Level lines were commenced and terminated at first or second order bench marks that were established and adjusted by the U.S. coast and Geodetic Survey to sea level. Errors of closure found by the level work were adjusted throughout the line, to the elevations published for the higher-order bench marks. The elevations of the temporary bench marks shown herein are of third-order accuracy.

Most of the bench marks listed in this table were established in the 1970's. Since then, some bridges have been abandoned, and a number of old bridges have been repaired or replaced. As a result, many of the original bench marks have been destroyed. The status of the original bench marks at intermediate points between the bridges is not known.

Three bridges on the lower West Nishnabotna River had new bench-mark elevations established for the 1987 flood profiles. During 1989, bridge deck and low-bridge chord elevations were surveyed, and low-water elevations were measured for those bridges indicated on the West and East Nishnabotna River profiles (figs. 11-27). Due to the loss of original bench marks, several supplemental bench-mark elevations were obtained from the aforementioned agencies, and several bridges will require level-line surveys to establish sea-level elevations. Bench marks associated with either of these conditions are noted in the following descriptions. Since bench marks are destroyed during time, the user should be cautious if the given bench-mark descriptions and elevations do not exactly match those in the field. 
The bench marks have been identified by an index number, which is composed of the Congressional township, range, section number and quarter-section in which they are located. The township and range numbers have been combined into a four-digit number, such as 7241 for township 72 north and range 41 west. This is followed by a dash and the section number in which the mark is located. Within the section, the quarter in which the mark is located is designated by $\mathrm{NE}, \mathrm{SE}, \mathrm{SW}$, or $\mathrm{NW}$. A number in parentheses, following this letter designation, indicates the numerical order among the marks located in that particular quarter. The index number describes the landline location of the mark without further reference in the body of the description.

Most of the bridges included in the following list had two original bench marks established for them. Several bridges have had additional bench marks added to them to supplement lost or destroyed marks, or to compensate for shifts in the position of the stream channel. Unless it is known that a bridge has been replaced, all known bench marks are included for a bridge. If a particular bench mark was not located during the 1989 survey work, a note to that effect is included in the description. For some bridges, landline locations may differ for the upstream or downstream, or left or right, parts of the bridge. Therefore, some bridges have bench marks with numerically separated index numbers. In such situations, a cross-reference is provided to enable the user to locate all the bench marks for a bridge.

Standard marks such as chiseled squares and crosses were used on concrete or steel. On trees or poles, a 20-penny pole spike driven through a short piece of 1/8-inch galvanized pipe and placed horizontally was used. Existing marks were used wherever available, and the agency responsible for the mark, when known, is indicated in the description. If the agency is unknown, the mark is indicated as found. Marks indicated as (Reference point) following the index number were established to permit water surface elevations to be determined by use of a tape and weight. The terms "right" and "left" in the descriptions were determined facing in the direction of the flow of the stream.

Additional information, if available, can be obtained by writing to: U.S. Geological Survey, 400 South Clinton Street, P.O. Box 1230, Iowa City, Iowa 52244 . 
6742-22 SE (1) - At Hamburg, on U.S. Highway 275 bridge over Nishnabotna River, at left downstream side of bridge, top of bolt head at left end of bridge, lst bolt from left.

Elev. 922.21 feet.

6742-22 SE (2) - At Hamburg, on U.S. Highway 275 bridge over Nishnabota River, at right downstream end of bridge, top of first bolt head from right. Elev. 922.28 feet.

6742-22 SE (3) - (Reference point) At Hamburg, on U.S. Highway 275 bridge over Nishnabotna River, on downstream guardrail at center of bridge, near vertical truss member, top of bolt. Elev. 931.68 feet.

6742-22 SE (4) - (Reference point) At Hamburg, on U.S. Highway 275 bridge over Nishnabotna River, on downstream guardrail at center of bridge, 7 feet left of vertical truss member, a filed arrow.

Elev. 931.46 feet.

6742-22 SW (1) - At Hamburg, at corner of Washington and G Streets, 40 feet west of U.S. Highway 275 , southeast corner of park, inside the sidewalk line, a standard U.S. Geological Survey tablet stamped "P 9 1933".

Elev. 914.253 feet.

6840-7 SW (1) - At Farragut, 90 feet west of the railroad station, 35 feet west of the centerline of main north-south street, 25 feet south of track and at west edge of sidewalk, a standard disk stamped "F 9 1933" set in top of a concrete post.

Elev. 961.363 feet.

6841-19 SW (1) - About 1 mile west of Riverton, on county road J46 bridge over West Nishnabotna River, on left upstream wingwall curb, an Iowa Highway Commission bench mark. Also see 6842-25 NE (1-6).

Elev. 935.64 feet.

6841-20 NE (1) - About 0.5 mile north of Riverton, on county road L68 bridge over East Nishnabotna River, on left downstream wingwall, a chiseled cross.

(Elevation to be surveyed.)

6841-20 NE (2) - (Reference point) About 0.5 mile north of Riverton, on county road L68 bridge over East Nishnabotna River, on downstream guardrail, between $23 \mathrm{rd}$ and 24 th guardrail posts from right end of bridge, a filed arrow.

(Elevation to be surveyed.)

6841-21 NW (1) - About 0.5 mile north of Riverton, on county road L68 bridge over East Nishnabotna River, on left upstream wingwall, a chiseled cross.

(Elevation to be surveyed.)

6841-29 NW (1) - At Riverton, on county road J46 bridge over East Nishnabotna River, on left downstream abutment, standard disk stamped "J 9 Reset 1975".

Elev. 936.509 feet.

6841-29 NW (2) - (Reference point) At Riverton, on county road J46 bridge over East Nishnabotna River, on 33rd guardrail post from left downstream end of bridge, a filed arrow.

Elev. 938.71 feet. 
6842-25 NE (1) - About 1 mile west of Riverton, on county road J46 bridge over West Nishnabotna River, on downstream wingwa11, at right end of bridge, a chiseled square. Also see 6841-19 SW (1).

E1ev. 937.41 feet.

6842-25 NE (2) - (Reference point) About 1 mile west of Riverton, on county road J46 bridge over West Nishnabotna River, on downstream guardrail, left of 19 th guardrail post from left end of bridge, a filed arrow. Also see 6841-19 SW (1). Elev. 937.76 feet.

6842-25 NE (3) - (Reference point) About 1 mile west of Riverton, on county road J46 bridge over West Nishnabotna River, on 19 th guardrail post from left downstream end of bridge, a filed arrow. Also see 6841-19 SW (1). E1ev. 937.60 feet.

6842-25 NE (4) - (Reference point) About 1 mile west of Riverton, on county road J46 bridge over West Nishnabotna River, on 22 nd guardrail post from left downstream end of bridge, a filed arrow. Also see 6841-19 SW (1).

E1ev. 937.61 feet.

6842-25 NE (5) - (Reference point) About 1 mile west of Riverton, on county road 346 bridge over West Nishnabotna River, on downstream guardrail, between 19 th and 20 th guardrail posts from left end of bridge, a filed arrow. Also see 6841-19 SW (1). Elev. 937.78 feet.

6842-25 NE (6) - About 1 mile west of Riverton, on county road J46 bridge over West Nishnabotna River, on top of downstream wingwall at right end of bridge, a steel disk with chiseled cross. (Unable to locate $6 / 12 / 89$. )

Elev. 930.99 feet.

6939-7 SE (1) - About 1 mile north of Shenandoah, on county road bridge over East Nishnabotna River, on curb at left downstream end of bridge, a chisled cross. Elev. 968.29 feet.

6939-7 SE (2) - (Reference point) About 1 mile north of Shenandoah, on county road bridge over East Nishnabotna River, on 3 rd guardrail post from left downstream pier, a chiseled arrow. Elev. 970.49 feet.

6939-7 SW (1) - About 1.5 miles north of Shenandoah, on railroad bridge over East Nishnabotna River, on left upstream side of bridge on $12 \mathrm{X}$ 12 inch horizontal piling, a bolt head with chiseled cross.

E1ev. 965.45 feet.

6939-7 SW (2) - (Reference point) About 1.5 miles north of Shenandoah, on railroad bridge over East Nishnabotna River, on downstream side of bridge at center of bridge, on top of bolt head with brace, a chiseled arrow.

Elev. 968.78 feet. 
6939-8 SE (1) - About 1.4 miles northeast along Burlington Northern Railroad from crossing with Colorado and Eastern Railroad in Shenandoah, 279 feet west of southwest corner of a large house which sits on a small knoll, 235 feet south of centerline of a private road crossing, 70 feet northwest of centerline of state Highway 48, 17 feet southeast of southeast rail of track, 1.4 feet south of a telephone pole and in line with row of telephone poles, a standard U.S. Geological Survey tablet stamped "Q 161 1949" set in top of a concrete post.

Elev. 973.089 feet.

6940-12 NE (1) - About 1.9 miles north of Shenandoah, at intersection of U.S. Highway 59 and Colorado and Eastern Railroad, at the south abutment, in the top of the curb at the southwest corner of the U.S. Highway 59 bridge, a standard U.S. Geological Survey tablet stamped "D 98 Reset 1958". Elev. 970.240 feet.

6940-13 NE (1) - About 1.5 miles north of Shenandoah, on U.S. Highway 59 bridge over East Nishnabotna River, on curb at left downstream end of bridge, an Iowa Highway Commission bench mark. Elev. 970.68 feet.

6940-13 NE (2) - (Reference point) About 1.5 miles north of Shenandoah, on U.S. Highway 59 bridge over East Nishnabotna River, on downstream guardrail post at center of bridge, a chiseled arrow. (Unable to locate $6 / 6 / 89$.)

Elev. 973.34 feet.

6940-13 NE (3) - (Reference point) About 1.5 miles north of Shenandoah, on U.S. Highway 59 bridge over East Nishnabotna River, on downstream curb, 197 feet from left end of bridge, a chiseled square.

Elev. 971.02 feet.

6940-13 SW (1) About 1 mile west of Shenandoah, on county road J32 bridge over East Nishnabotna River, on right upstream corner of bridge wingwal1, an Iowa Highway Commission bench mark. Elevation obtained from Iowa Dept. of Transportation. Elev. 968.01 feet.

6940-14 SW (1) - About 2.2 miles west on county road J46 from intersection with State Highway 48 at west edge Shenandoah, set in top of west end of north headwall of a 4 foot concrete box culvert, a standard U.S. Geological Survey tablet stamped "J 156 1949". Elev. 1001.212 feet.

6940-24 NW (1) - About 1 mile west of Shenandoah, on county road J32 bridge over East Nishnabotna River, on abutment at left downstream end of bridge, at end of wingwall, an Iowa Highway Commission bench mark. Elevation established from 6940-13 SW (1). Elev. 968.21 feet.

6940-24 NW (2) - (Reference point) About 1 mile west of Shenandoah, on county road J32 bridge over East Nishnabotna River, on downstream concrete guardrail at center of bridge, a chiseled arrow. Elevation established from 6940-13 SW (1). Elev. 972.45 feet. 
6940-24 NW (3) About 1 mile west of Shenandoah, on county road J32 bridge over East Nishnabotna River, on downstream concrete guardrail, 6.5 feet from left end of bridge guardrail, an Iowa Highway Commission bench mark. Elevation established from 6940-13 SW (1).

Elev. 971.01 feet.

6940-24 NW (4) - (Reference point) About 1 mile west of Shenandoah, on county road J32 bridge over East Nishnabotna River, on downstream guardrail, 121 feet from left end of bridge, a chiseled square. Elevation established from 6940-13 SW (1). Elev. 972.27 feet.

6940-28 SE (1) - About 2.5 miles northeast of Farragut, on State Highway 2 bridge over East Nishnabotna River, on right upstream abutment curb, an Iowa Highway Commission bench mark. Elevation obtained from Iowa Dept. of Transportation. Elev. 957.61 feet.

6940-28 SE (2) - (Reference point) About 2.5 miles northeast of Farragut, on State Highway 2 bridge over East Nishnabotna River, on downstream curb, between 6 th and 7 th guardrail posts from right pier, a chiseled square. Elevation established from 6940-28 SE (1).

E1ev. 958.14 feet.

6940-30 SE (1) - About 1.2 miles north of Farragut, on county road M16 bridge over East Nishnabotna River, on abutment at left downstream end of bridge, an U.S. Army Corps of Engineers control plate.

Elev. 953.56 feet.

6940-30 SE (2) - (Reference point) About 1.2 miles north of Farragut, on county road M16 bridge over East Nishnabotna River, on downstream curb, at 18 th guardrail post from left end of bridge, a chiseled square.

E1ev. 953.96 feet.

6940-31 NE (1) - About 0.8 mile north of Farragut, along county road M16, on west side of highway, west of cemetary, in telephone pole east of building, a pole spike and collar. Elev. 952.14 feet.

6941-8 NW (1) - About 4 miles northeast of Sidney, on county road J26 bridge over West Nishnabotna River, on downstream wingwall at right end of bridge, a chiseled square. Elev. 948.62 feet.

6941-8 NW (2) - (Reference point) About 4 miles northeast of Sidney, on county road J26 bridge over West Nishnabotna River, on 25 th guardrail post from right downstream end of bridge, a filed arrow.

E1ev. 950.97 feet.

6941-8 NW (3) - (Reference point) About 4 miles northeast of Sidney, on county road J26 bridge over West Nishnabotna River, on downstream guardrail, near 35 th guardrail post from right end of bridge, a filed arrow.

Elev. 950.96 feet.

6941-8 NW (4) - About 4 miles northeast of Sidney, on county road J26 bridge over West Nishnabotna River, on 1st guardrail post from right downstream end of bridge, a chiseled mark. E1ev. 950.32 feet. 
6941-9 NW (1) - About 4.5 miles northeast of Sidney and 1 mile west of county road L68, at southeast corner of intersection of T-road south, in north side of power pole 1 foot above ground, a pole spike and collar.

Elev. 938.80 feet.

6941-10 NW (1) - From Randolph, 5.2 miles south along county road L68, 0.3 mile north of Walnut Creek, 176 feet east of county road centerline, 41 feet north of driveway centerline, a standard disk set in concrete post and stamped "S 157 1949".

Elev. 941.327 feet.

6941-29 SW (1) - About 2 miles east of Sidney, on State Highway 2 bridge over West Nishnabotna River, on left downstream wingwa11, an Iowa Highway Commission bench mark. Elev. 946.78 feet.

6941-30 NE (1) - About 2 miles east of Sidney, on State Highway 2 bridge over West Nishnabotna River, on end of right downstream abutment, a disk with chiseled cross. Elev. 946.62 feet.

6941-30 NE (2) - (Reference point) About 2 miles east of Sidney, on State Highway 2 bridge over West Nishnabotna River, on downstream curb, at 26 th guardrail post from right end bridge, a chiseled arrow. Also a chiseled square on downstream curb at 27 th guardrail post from right end bridge, at same elevation. Elev. 947.89 feet.

6941-30 NE (3) - About 2 miles east of Sidney, on State Highway 2 bridge over West Nishnabotna River, on right upstream abutment curb, an Iowa Highway Commission bench mark.

Elev. 946.73 feet.

7039-1 SW (1) - About 2 miles southwest of Coburg, on county road J14 bridge over East Nishnabotna River, on left upstream abutment curb, a bolt in concrete. (Elevation to be surveyed.)

7039-12 SE (1) - About 2.9 miles southwest along railroad from station at Colburg, 31 feet west of east right-of-way fence, 18.4 feet east of east rail of track, 3.7 feet north of a telephone pole, and in line with row of telephone poles, about 2 feet lower than track, 1.2 feet south of reference post, set in top of concrete post projecting 0.3 foot above ground, a standard U.S. Geological Survey tablet stamped "P 161 1949".

Elev. 997.964 feet.

7039-12 NW (1) - (Reference point) About 2 miles southwest of Coburg, on county road J14 bridge over East Nishnabotna River, on downstream guardrail, between 16 th and 17 th guardrail posts from right end of bridge, a filed arrow.

(Elevation to be surveyed.)

7039-22 NE (1) - (Reference point) About 1 mile northeast of Essex, on county road M41 bridge over East Nishnabotna River, on downstream guardail, between 21 st and 22 nd guardrail posts, a chiseled arrow. E1ev. 995.17 feet.

7039-23 NW (1) - About 1 mile northwest of Essex, on county road M41 bridge over East Nishnabotna River, on curb behind left upstream wingwall, a bolt head.

Elev. 992.45 feet. 
7039-26 NW (1) - About 0.8 mile northeast along railroad from station at Essex, on a $5 \times 20$ foot concrete culvert along State Highway 48, opposite a small railroad trestle, set in top of southwest end of the northwest headwal1, a standard tablet stamped "M 161 1949".

Elev. 998.853 feet.

7039-27 SE (1) - At Essex, northwest of railroad station, at intersection of county road M4l, at southwest corner of intersection, in north side of power pole, a pole spike and collar. Elev. 993.61 feet.

7039-27 NW (1) - About 0.3 mile west of Essex, on county road bridge over East Nishnabotna River, on curb at right downstream end of bridge, an Iowa Highway Commission bench mark. Elev. 984.30 feet.

7039-27 NW (2) - (Reference point) About 0.3 mile west of Essex, on county road bridge over East Nishnabotna River, on downstream guardrail post at center of bridge, a chiseled arrow.

Elev. 986.62 feet.

7039-27 NW (3) - (Reference point) About 0.3 mile west of Essex, on county road bridge over East Nishnabotna River, on downstream guardrail, between 8 th and 9 th guardrail posts, a filed arrow.

Elev. 986.58 feet.

7041-8 SE (1) - About 0.5 mile west of Randolph, on State Highway 184 bridge over West Nishnabotna River, on upstream curb near left end of bridge, a standard U.S. Coast and Geodetic Survey brass tablet stamped "AA-158, 1973".

Elev. 967.309 feet.

7041-17 NW (1) - About 0.5 mile west of Randolph, on State Highway 184 bridge over West Nishnabotna River, on downstream curb near right end of bridge, an Iowa Highway Commission bench mark.

Elev. 967.24 feet.

7041-31 NW (1) - About 5.5 miles north of Sidney, at intersection of county roads at northeast corner of section 31 , at southeast corner of intersection, in north side of power pole 1 foot above ground, a pole spike and collar.

Elev. 1042.31 feet.

7041-32 NW (1) - About 3 miles south of Randolph, on county road J22 bridge over West Nishnabotna River, on downstream guardrail, on top of first bolt from left end of guardrail.

Elev. 958.42 feet.

7041-32 NW (2) - (Reference point) About 3 miles south of Randolph, on county road J22 bridge over West Nishnabotna River, on downstream guardrail at center of bridge, 3 filed marks. Elev. 959.29 feet.

7041-32 NW (3) - About 3 miles south of Randolph, on county road J22 bridge over West Nishnabotna River, on downstream wingwall at left end bridge, a chiseled square.

E1ev. 956.22 feet.

7042-26 SE (1) - About 6 miles north of Sidney, near southeast corner of section 26, 34 feet north and 236 feet west of $T$-road north, 11 feet east of field entrance, 1 foot south of fence line, standard disk stamped "95 RC 1955 1106".

Elev. 1106.103 feet. 
7138-18 SE (1) - About 1.9 miles northeast along the Burlington and Northern Railroad from station at Coburg, at a road crossing, 855 feet north of railroad trestle, 129 feet south of centerline of east-west road, 10.7 feet west of west rail, 2.0 feet lower than railroad, set in top of center of west headwall of a $9 \times 3$ foot concrete culvert, a standard U.S. Geological Survey tablet stamped "G $1611949 "$.

Elev. 1011.442 feet.

7138-19 NW (1) - About 1.5 miles north of Coburg, on county road H46 bridge over East Nishnabotna River, on top of first bolt on guardrail from left downstream end of bridge, a chiseled cross on bolt.

Elev. 1020.64 feet.

7138-19 NW (2) - (Reference point) About 1.5 miles north of Coburg, on county road H46 bridge over East Nishnabotna River, on downstream guardrail, centered between piers, a chiseled arrow.

Elev. 1020.89 feet.

7138-30 SW (1) - At Coburg, 153 feet northeast of station at Coburg, 49 feet north of centerline of road, 33 feet east of east rail of main track, 9.3 feet northwest of a telephone pole, 1.0 foot west of reference post, set in top of a concrete post projecting 0.3 foot above ground, a standard U.S. Geological Survey tablet stamped "M 98 1935".

Elev. 1003.600 feet.

7139-36 NE (1) - About 0.5 mile west of Coburg, on county road H54 bridge over East Nishnabotna River, on left downstream abutment, a chiseled square.

Elev. 1009.58 feet.

7139-36 NE (2) - (Reference point) About $0.5 \mathrm{mile}$ west of Coburg, on county road H54 bridge over East Nishnabotna River, on 2nd guardrail post right of left downstream pier, a chiseled arrow.

Elev. 1013.53 feet.

7141-2 NW (1) - About 3 miles northwest of strahan, on county road bridge over West Nishnabotna River, on downstream end of left pier, on top of left downstream corner of I-beam. Also see 7241-35 SW (1).

Elev. 989.60 feet.

7141-2 NW (2) - (Reference point) About 3 miles northwest of Strahan, on county road bridge over West Nishnabotna River, on downstream guardrail at center of bridge, on horizontal bolt, a chiseled arrow. Also see 7241-35 SW (1).

Elev. 995.30 feet.

7141-2 NW (3) - About 3 miles southeast of Malvern, on railroad bridge over West Nishnabotna River, on top of left downstream abutment, a chiseled square.

Elev. 987.10 feet.

7141-2 NW (4) - (Reference point) About 3 miles southeast of Malvern, on railroad bridge over West Nishnabotna River, at downstream side of bridge at center of bridge, on top of bolt head at vertical upright, a chiseled cross.

Elev. 991.84 feet. 
7141-4 NE (1) - From Malvern, 2.2 miles southeast along railroad track from station, at a road crossing, 51 feet west of centerline of road, 42 feet south of centerline of track, 7 feet southeast of a pole, 4 feet north of a fence, and about 2.5 feet lower than the track, a standard disk stamped "S 971935 " set in top of a concrete post.

Elev. 1001.373 feet.

7141-16 NE (1) - About 3.5 miles south of Malvern, on county road L68 bridge over West Nishnabotna River, on top of left downstream pier, a chiseled square. E1ev. 974.83 feet.

7141-16 NE (2) - (Reference point) About 3.5 miles south of Malvern, on county road L68 bridge over West Nishnabotna River, on downstream guardrail, between 2 nd and $3 \mathrm{rd}$ vertical truss member from left end of bridge, a filed arrow.

Elev. 982.81 feet.

7141-22 NW (1) - About 3 miles west of Strahan, 42 feet north of intersection of county roads $\mathrm{L} 68$ and $\mathrm{H46}$, at $10 \mathrm{X} 12$ foot concrete culvert, on top of south end of east culvert headwall, a standard disk stamped "FF 1581949 ".

E1ev. 973.597 feet.

7238-8 SE (1) - (Reference point) About 1.5 miles north of Red Oak, on State Highway 48 bridge over East Nishnabotna River, on downstream curb, at 2nd guardrail post from right pier, a chiseled square.

E1ev. 1051.83 feet.

7238-9 SW (1) - About 1.5 miles north of Red Oak, on State Highway 48 bridge over East Nishnabotna River, on left upstream abutment, an Iowa Highway Commission bench mark. Elev. 1049.50 feet.

7238-9 SW (2) - About 1.5 miles north of Red Oak, on State Highway 48 bridge over East Nishnabotna River, on upstream curb at left end of bridge, an Iowa Highway Commission bench mark. (Unable to locate $6 / 2 / 89$.)

Elev. 1054.88 feet.

7238-16 NW (1) - About 1.3 miles north along State Highway 48 from U.S. Highway 34 at Red Oak, at railroad signa1, on top of threaded bolt on northwest corner of railroad signal base, a chiseled cross.

Elev. 1053.54 feet.

7238-20 NE (1) - At Red Oak, 0.4 mile west of intersection of U.S.Highways 34 and 48, 10 feet west of railroad track, at railroad crossing signal south of U.S. Highway 34, on top of threaded bolt on northeast corner of railroad signal base, a chiseled cross. Elev. 1036.63 feet.

7238-20 SE (1) - At Red Oak, on county road H34 bridge over East Nishnabotna River, on upstream end of right pier, a brass disk. Also see $7238-29$ SE (1).

Elev. 1033.60 feet.

7238-20 NW (1) - About 1 mile northwest of Red Oak, on U.S. Highway 34 bridge over East Nishnabotna River, on left downstream abutment, an Iowa Highway Commission bench mark.

Elev. 1041.91 feet. 
7238-20 NW (2) - (Reference point) About 1 mile northwest of Red Oak, on U.S. Highway 34 bridge over East Nishnabotna River, on downstream curb at left pier, a chiseled square. Elev. 1042.60 feet.

7238-29 SE (1) - (Reference point) At Red Oak, on county road H34 bridge over East Nishnabotna River, on downstream guardrail, near 2nd vertical truss member from right end of bridge, a filed arrow. Also see 7238-20 SE (1).

Elev. 1041.68 feet.

7238-29 SE (2) - At west edge of Red Oak, on Coolbaugh Street bridge over East Nishnabotna River, on left upstream concrete abutment, a standard U.S. Army Corps of Engineers disk.

Elev. 1032.74 feet.

7239-19 NW (1) - About 1 mile northeast of Emerson, on U.S. Highway 34 bridge over Indian Creek, on right downstream wingwall, an Iowa Highway Commission bench mark.

Elev. 1068.08 feet.

7239-19 NW (2) - (Reference point) About 1 mile northeast of Emerson, on U.S. Highway 34 bridge over Indian Creek, on downstream curb, 70 feet from left abutment, a $3 / 8$ inch bolt.

Elev. 1068.67 feet.

7240-5 SW (1) - About 2.5 miles north of Hastings, on county road M16 about 0.2 mile north of county road $\mathrm{H} 26$, about 0.1 mile north of farmhouse, 35 feet east of centerline of road, 28 feet north of centerline of field entrance road, 1 foot west of east right of way fence, 1.3 feet south of a reference post, a standard disk stamped "Z $1471949 "$ set in top of a concrete post. Elev. 1032.688 feet.

7240-7 NW (1) - About 2 miles north of Hastings, on county road $\mathrm{H} 26$ bridge over West Nishnabotna River, near left downstream corner of right downstream pier, a chiseled square.

Elev. 1011.33 feet.

7240-7 NW (2) - (Reference point) About 2 miles north of Hastings, county road H26 bridge over West Nishnabotna River, on downstream guardrail, at 2 nd vertical truss member from right end of bridge, a filed arrow.

Elev. 1016.99 feet.

7240-19 NW (1) - At Hastings, on county road Ml6 bridge over Indian Creek, about 250 feet south of U.S. Highway 34, on right upstream abutment about 0.5 foot above road level, a standard disk stamped "W 147 1949".

Elev. 1008.236 feet.

7240-20 SE (1) - (Reference Point) About 3 miles west of Emerson, on county road bridge over Indian Creek, on 8 th guardail post from right downstream abutment, 3 chisel marks.

E1ev. 1026.05 feet.

7240-21 SE (1) - About 2 miles west of Emerson, on county road M21 bridge over Indian Creek, on end of left downstream wingwall, top of nut.

Elev. 1040.89 feet.

7240-21 SE (2) - (Reference point) About 2 miles west of Emerson, on county road M21 bridge over Indian Creek, on downstream guardrail, 40 feet from right abutment, a chiseled ledge.

Elev. 1040.40 feet. 
7240-21 SW (1) - About 3 miles west of Emerson, on county road bridge over Indian Creek, on top of right upstream wingpost, a painted square.

Elev. 1026.63 feet.

7240-23 SE (1) - At west edge of Emerson, at northwest corner of Harris and Morton Streets, on fire hydrant, top of nut. Elev. 1073.35 feet.

7240-24 SW (1) - At Emerson, at northeast corner of Howland and Morton Streets, on fire hydrant, "O" in "IOWA". 1055.92 feet.

7240-24 SW (2) - (Reference point) Near east edge of Emerson, on county road $\mathrm{H} 34$ bridge over Indian Creek, on center of downstream guardrail, a chiseled arrow.

Elev. 1060.00 feet.

7240-24 SW (3) - Near east edge of Emerson, on county road H34 bridge over Indian Creek, on left downstream abutment, a chiseled square.

E1ev. 1057.10 feet.

7240-24 NW (1) - About 0.5 mile northeast of Emerson and about 0.5 mile downstream from U.S. Highway 34 , on unimproved county road culvert over Indian Creek, on top of culvert. Elev. 1064.34 feet.

7240-26 NE (1) - Near southwest corner of Emerson, on county road bridge over Indian Creek, on right downstream curb, a chiseled cross.

Elev. 1050.75 feet.

7240-26 NE (2) - (Reference point) Near southwest corner of Emerson, on county road bridge over Indian Creek, on center downstream guardrail post, 3 chise1 marks.

Elev. 1052.81 feet.

7240-26 NW (1) - About 1 mile west of Emerson, on county road concrete culvert over Indian Creek, on downstream headwall, a painted square.

Elev. 1050.02 feet.

7240-26 NW (2) - About 1 mile west of Emerson, on county road bridge over Indian Creek, on right upstream abutment pile cap, a railroad spike. Elev. 1041.07 feet.

7240-27 NE (1) - (Reference point) About 1 mile west of Emerson, on county road bridge over Indian Creek, on 3rd angle iron support from right downstream abutment, 3 chisel marks.

Elev. 1046.33 feet.

7240-30 SW (1) - About 1.5 miles south of Hastings, at intersection of county roads $\mathrm{M} 16$ and $\mathrm{H} 38$, in northeast corner of intersection, 78 feet east of centerline of county road M16, 30 feet north of centerline of county road H38, 44 feet east of a power pole, 1 foot south of the north right-of-way fence, 1.4 feet west of a witness post, a standard disk stamped "U 1471949 " set in top of a concrete post.

Elev. 1077.724 feet.

7241-13 SE (1) - (Reference point) Near northwest corner of Hastings, on U.S. Highway 34 bridge over Indian Creek, on center of downstream guardrail, 3 chisel marks.

Elev. 1010.50 feet. 
7241-23 SE (1) - About 1.5 miles west of Hastings, on railroad bridge over West Nishnabotna River, on left downstream wingwall, a chiseled square.

Elev. 1002.92 feet.

7241-23 SE (2) - (Reference point) About 1.5 miles west of Hastings, on railroad bridge over West Nishnabotna River, at center of downstream side of bridge, on top of a bolt head across from a guardrail post, a chiseled arrow. Elev. 1007.50 feet.

7241-24 NE (1) - Near north edge of Hastings, on U.S. Highway 34 bridge over Indian Creek, on left upstream wingwall, a chiseled cross.

Elev. 1008.08 feet.

7241-24 NE (2) - (Reference point) Near north edge of Hastings, on county road M16 bridge over Indian Creek, on downstream guardrail, about 25 feet from right pier, a chiseled arrow. Elev. 1011.25 feet.

7241-24 NW (1) - About 0.5 mile west of Hastings, on U.S. Highway 34 bridge over West Nishnabotna River, on right upstream wingwall guardrail, an Iowa Highway Commission bench mark (7/90). Elev. 1009.57 feet.

7241-24 NW (2) - (Reference point) About 0.5 mile west of Hastings, on U.S. Highway 34 bridge over West Nishnabotna River, on center of downstream guardrail, a chiseled square (7/90). Elev. 1009.93 feet.

7241-24 NW (3) - About 0.5 mile west of Hastings, on U.S. Highway 34 bridge over West Nishnabotna River, on left downstream wingwall guardrail, an Iowa Highway Commission bench mark (7/90). Elev. 1009.84 feet.

7241-35 NE (1) - About 2 miles southwest of Hastings, on county road H38 bridge over West Nishnabotna River, on end of left downstream abutment, a chiseled square. Elev. 996.02 feet.

7241-35 NE (2) - (Reference point) About 2 miles southwest of Hastings, on county road H38 bridge over West Nishnabotna River, on downstream guardrail, at 1st vertical truss member from left end of bridge, a filed arrow.

E1ev. 1002.18 feet.

7241-35 SW (1) - About 3 miles northwest of Strahan, on county road bridge over West Nishnabotna River, on upstream guardrail at right end of bridge, on first bolt head from end of guardrail, a chiseled cross. Also see 7141-2 NW (1-2). Elev. 995.47 feet.

7338-1 SE (1) - At Elliott, southeast of railroad, 42 feet south of south curb of street and 36 feet west of east rail of main track, a standard U.S. Geological Survey tablet stamped "Y 98 1935" set in a concrete post. Elev. 1075.415 feet.

7338-2 SE (1) - About 0.5 mile west of Elliott, on State Highway 48 bridge over East Nishnabotna River, on right upstream abutment, an Iowa Highway Commission bench mark.

Elev. 1081.33 feet. 
7338-11 NE (1) -(Reference point) About 0.5 mile west of Elliott, on State Highway 48 bridge over East Nishnabotna River, on downstream curb at center guardrail post, a chiseled square. Elev. 1079.21 feet.

7338-12 NW (1) - South of Elliott, at intersection of State Highway 48 and county road M55, southwest of intersection, in tallest of 2 telephone poles, in north side of pole, a pole spike and collar.

Elev. 1070.52 feet.

7338-14 SE (1) - About 2 miles north of stennett, on railroad bridge over creek, over right upstream pile cap, a 1.5 inch drift pin.

Elev. 1059.12 feet.

7338-14 SE (2) - About 2 miles north of Stennett, at a private-road crossing, 48 feet southeast of centerline of railroad track, 21 feet northeast of centerline of private road, 57 feet southwest of a pole, 5 feet northwest of right-of-way fence, a standard disk stamped

"W 98 1935" set in top of a concrete post. Elev. 1059.827 feet.

7338-23 SW (1) - About 1 mile north of Stennett, on dead-end road, in pole west of railroad, nail and disk in pole 1 foot above ground.

Elev. 1060.51 feet.

7338-34 NW (1) - About 0.5 mile southwest of Stennett, on county road H20 bridge over East Nishnabotna River, on curb at left downstream end of bridge, a chiseled cross.

Elev. 1058.64 feet.

7338-34 NW (2) - (Reference point) About 0.5 mile southwest of Stennett, on county road $\mathrm{H} 20$ bridge over East Nishnabotna River, on downstream guardrail, centered between 2 left piers, a chiseled arrow.

Elev. 1060.97 feet.

7338-34 NW (3) - About 0.5 mile southwest of Stennett, on county road $\mathrm{H} 20$ bridge over East Nishnabotna River, on downstream wingwall curb, at left end of bridge, a chiseled square. Elev. 1058.60 feet.

7340-3 SW (1) - About 1 mile northwest of Henderson, on county road H12 bridge over West Nishnabotna River, at left end of upstream guardrail, a chiseled square in concrete. Also see 7340-10 NW (1-4).

Elev. 1045.38 feet.

7340-3 NW (1) - About 1.2 miles north of Henderson, on county road M21 bridge over small creek, on left downstream side, a chiseled square.

Elev. 1044.78 feet.

7340-10 NW (1) - About 1 mile northwest of Henderson, on county road H12 bridge over West Nishnabotna River, at right end of downstream guardrail, a chiseled square in concrete. Also see 7340-3 SW (1).

Elev. 1043.65 feet.

7340-10 NW (2) - (Reference point) About 1 mile northwest of Henderson, on county road H12 bridge over West Nishnabotna River, on downstream guardrail, 137 feet east of 7340-10 NW (1), a filed arrow. Also see 7340-3 SW (1).

Elev. 1044.33 feet. 
7340-10 NW (3)- About 1 mile northwest of Henderson, on county road $\mathrm{H} 12$ bridge over West Nishnabotna River, at left end of downstream guardrail, a chiseled square in concrete. Also see 7340-3 SW (1).

Elev. 1045.42 feet.

7340-10 NW (4) - About 1 mile northwest of Henderson, on county road H12 bridge over West Nishnabotna River, on concrete centerline of bridge deck, 143 feet east of 7340-10 NW (1), a chiseled cross. Also see 7340-3 SW (1).

Elev. 1041.90 feet.

7340-30 SE (1) - About 4 miles north of Hastings, on county road M16 bridge over West Nishnabotna River, on downstream end of right pier, a standard tablet stamped "C 148 1949". Elev. 1014.047 feet.

7340-30 SE (2) - (Reference point) About 4 miles north of Hastings, on county road M16 bridge over West Nishnabotna River, on downstream guardrail, at 5 th vertical truss member from left end of bridge, a filed arrow.

Elev. 1022.64 feet.

7437-6 SE (1) - At Griswold, at the public school, 102 feet west of main entrance, 1 foot southwest of flag pole, a standard U.S. Geological Survey tablet stamped "Griswold 1934". Elev 1103.147 feet.

7438-1 SE (1) - About 1 mile west of Griswold, on State Highway 92 bridge over East Nishnabotna River, on right downstream abutment, an Iowa Highway Commission bench mark. (Unable to locate 6/1/89.)

Elev. 1100.77 feet.

7438-1 SE (2) - (Reference point) About 1 mile west of Griswold, on State Highway 92 bridge over East Nishnabotna River, on 10th guardrail post from right downstream end of bridge, a chiseled arrow.

Elev. 1103.42 feet.

7438-1 SE (3) - About 1 mile west of Griswold, on State Highway 92 bridge over East Nishnabotna River, on right downstream wingwall, a chiseled cross.

(Elevation to be surveyed.)

7438-36 NE (1) - About 1.7 miles north of Elliott, along Chicago, Burlington and Quincy Railroad, southwest of county road G66 crossing, 34.5 feet south of centerline of county road G66 and 35.5 feet west of centerline of tracks, a standard U.S. Geological Survey tablet stamped "Z 98 1935" set in a concrete post. Elev. 1086.195 feet.

7438-36 NW (1) - About 1.5 miles north of Elliott, on county road G66 bridge over East Nishnabotna River, on right downstream wingwall, top of bolt head.

Elev. 1081.38 feet.

7438-36 NW (2) - (Reference point) About 1.5 miles north of Elliott, on county road G66 bridge over East Nishnabotna River, on downstream guardrail, between 24 th and 25 th guardrail posts from right end of bridge, a chiseled arrow. (Unable to locate 6/2/89.)

Elev. 1083.62 feet. 
7438-36 NW (3) - (Reference point) About 1.5 miles north of Elliott, on county road G66 bridge over East Nishnabotna River, on downstream curb, between 14 th and 15 th guardrail posts from left end of bridge, a chiseled square.

Elev. 1081.77 feet.

7440-3 NE (1) - At northwest edge of Carson, on county road bridge over West Nishnabotna River, on right downstream pier, a chiseled square. Elev. 1063.66 feet.

7440-3 NE (2) - (Reference point) At northwest edge of Carson, on county road bridge over West Nishnabotna River, on 3rd vertical truss member from right downstream end of bridge, a filed mark.

Elev. 1070.28 feet.

7440-10 NE (1) - At Carson, at intersection of State Highway 92 and Commercial street, southwest of intersection, 57 feet south of centerline of State Highway 92, 47 feet east of east rail of the railroad tracks, 1 foot northwest of a driven railroad rail projecting 0.6 foot above ground, a standard disk stamped "H 149 reset 1955 " set in top of a concrete post. Elev. 1067.428 feet.

7440-10 NE (2) - At Carson, on State Highway 92 bridge over West Nishnabotna River, on left downstream abutment, a steel pin.

Elev. 1076.38 feet.

7440-10 NE (3) - (Reference point) At Carson, on State Highway 92 bridge over West Nishnabotna River, on downstream curb, at 20 th guardrail post from left end of bridge, a chiseled square. Elev. 1081.19 feet.

7440-21 SE (1) - About 1 mile west of Macedonia, on county road G66 bridge over West Nishnabotna River, on top of left downstream wingwall, a chiseled square.

Elev. 1057.70 feet.

7440-21 SE (2) - (Reference point) About 1 mile west of Macedonia, on county road G66 bridge over West Nishnabotna River, on 22nd guardrail post from left downstream end of bridge, a filed arrow.

Elev. 1060.34 feet.

7440-22 SE (1) - At Macedonia, at intersection of county roads M21 and G66, 29 feet east and 55 feet south of intersection, at base of power pole, a pole spike and collar. Elev. 1105.57 feet.

7440-27 SE (1) - About 1 mile south of Macedonia, on county road M21 culvert over drainage ditch, in center of east headwall, an Iowa Highway Commission bench mark.

Elev. 1043.55 feet.

7537-3 SE (1) - About 0.5 mile north of Lewis, on U.S. Highway 6 bridge over East Nishnabotna River, on right upstream wingwall, an Iowa Highway Commission bench mark. Also see 7537-10 NE (1).

Elev. 1132.95 feet. 
7537-4 SW (1) - About 2 miles west of Lewis, along U.S. Highway 6, 297 feet north and 34 feet east of intersection with north-south county road, north of a telephone pole, 2 feet north of a witness post, a standard U.S. Geological Survey tablet stamped " 3 PJH 1968".

Elev. 1209.445 feet.

7537-9 NW (1) - About 2 miles northwest of Lewis, on U.S. Highway 6 bridge over small creek, on left upstream abutment, a chiseled square.

Elev. 1146.22 feet.

7537-10 NE (1) - (Reference point) About 0.5 mile north of Lewis, on U.S. Highway 6 bridge over East Nishnabotna River, on downstream curb, near 20 th guardrail post from right end of bridge, a chiseled square. Also see 7537-3 SE (1).

E1ev. 1132.66 feet.

7537-10 SW (1) - About 0.2 mile west of Lewis, on county road bridge over East Nishnabotna River, on I-beam at right downstream end of truss, a chiseled arrow.

Elev. 1122.78 feet.

7537-10 SW (2) - (Reference point) About 0.2 mile west of Lewis, on county road bridge over East Nishnabotna River, on downstream guardrail, centered between $3 \mathrm{rd}$ and 4 th vertical truss members from right end of bridge, a chiseled arrow. E1ev. 1127.78 feet.

7537-18 SE (1) - About 3.5 miles north of Griswold, on State Highway 48 bridge over East Nishnabotna River, on top of right downstream abutment, a chiseled square.

E1ev. 1116.39 feet.

7537-19 NE (1) - (Reference point) About 3.5 miles north of Griswold, on State Highway 48 bridge over East Nishnabotna River, on downstream curb, at center guardrail post, a chiseled square.

Elev. 1122.01 feet.

7537-20 SW (1) - About 2.5 miles north of Griswold, along State Highway 48, 27 feet north and 45 feet west of $T$-road east, on top of north end of a 1.5 foot corrugated pipe culvert, a punched hole "UE 4 PJH A".

E1ev. 1124.71 feet.

7537-29 SW (1) - About 1.5 miles north of Griswold, on State Highway 48, 78 feet north, 70.5 feet east and 4.3 feet below crossroads, about 4 feet southeast of power pole, a standard U.S. Geological Survey tablet stamped "5 PJH 1968".

Elev. 1110.912 feet.

7537-30 NE (1) - About 2.5 miles north of Griswold, on county road bridge over East Nishnabotna River, above first pier from right downstream side of bridge, top of right downstream bolt. Elev. 1104.43 feet.

7537-30 NE (2) - (Reference point) About 2.5 miles north of Griswold, on county road bridge over East Nishnabotna River, on downstream guardrail, centered between 4 th and 5 th vertical truss members from right end of bridge, a chiseled arrow. Elev. 1108.35 feet. 
7537-30 NE (3) - About 2.5 miles north of Griswold, on county road bridge over East Nishnabotna River, above first pier from right downstream side of bridge, top of left downstream bolt, a filed cross.

Elev. 1104.43 feet.

7537-30 NE (4) - (Reference point) About 2.5 miles north of Griswold, on county road bridge over East Nishnabotna River, on downstream guardrail, between 1 st and 2nd vertical truss members from left end of bridge, a filed arrow.

Elev. 1108.26 feet.

7537-35 SW (1) - About 1.5 miles northwest of Lewis, at intersection of U.S. Highway 6 and north-south county road, at northeast corner of intersection, in south side of telephone pole, a pole spike and collar.

Elev. 1198.36 feet.

7540-11 SE (1) - At Oakland, on county road G42 bridge over West Nishnabotna River, on left downstream wingwall, a chiseled square.

Elev. 1089.72 feet.

7540-11 SE (2) - (Reference point) At Oakland, on county road G42 bridge over West Nishnabotna River, on 25 th guardrail post from left

downstream end of bridge, a filed arrow. Elev. 1092.98 feet.

7540-11 SE (3) - (Reference point) At Oakland, on county road G42 bridge over West Nishnabotna River, on 13 th guardrail post from left

downstream end of bridge, a filed arrow. Elev. 1092.55 feet.

7540-12 SW (1) - At Oakland, on U.S. Highway 59 at the old Citizens State Bank building, in the east face at the southeast corner of the building, a standard tablet stamped "P 147 1949".

Elev. 1102.619 feet.

7540-14 SE (1) - About 0.5 mile south of Oakland, on U.S. Highways 6 and $59,0.2$ mile north of junction of U.S. Highways 6 and 59 , about 150 feet south of a large red stone farmhouse on west side of highway, 42 feet west of centerline of highway, 19 feet northeast of centerline of a gate, 3 feet west of a corner fence post, 3 feet east of a telephone pole, 1.6 feet south of a fence, a standard tablet stamped "R 149 1949" set in top of a concrete post.

E1ev. 1093.059 feet.

7540-14 SE (2) - About 1 mile south of Oakland, on U.S. Highway 6 bridge over West Nishnabotna River, on upstream side of right upstream wingwal1, top of large nut. Elevation established from 7540-23 NE (1).

Elev. 1086.78 feet.

7540-23 NE (1) - About 1 mile south of Oakland, on U.S. Highway 6 bridge over West Nishnabotna River, on top of corner of right downstream wingpost, an Iowa Highway Commission bench mark. Elevation obtained from Iowa Dept. of Transportation.

E1ev. 1087.52 feet.

7540-23 NE (2) - (Reference point) About 1 mile south of Oakland, on U.S. Highway 6 bridge over West Nishnabotna River, on downstream concrete guardrail, 148 feet from left end of bridge, a chiseled square. Elevation established from 7540-23 NE (1).

Elev. 1088.01 feet. 
7636-6 SE (1) - At Atlantic, on State Highway 83 bridge over East Nishnabotna River, on curb at right upstream end of bridge, a standard U.S. Geological Survey tablet stamped "X 162 1950". (Unable to locate $6 / 1 / 89$.) Elev. 1152.98 feet.

7636-6 SE (2) At Atlantic, on State Highway 83 bridge over East Nishnabotna River, on top of wingwall, at right downstream end of bridge, a standard National Geodetic Survey brass tablet. Elevation obtained from Iowa Dept. of Transportation.

Elev. 1155.112 feet.

7636-6 SE (3) - (Reference point) At Atlantic, on State Highway 83 bridge over East Nishnabotna River, on 15th guardrail post from right downstream end of bridge, a filed arrow. Elevation established from 7636-6 SE (2). Elev. 1155.17 feet.

7636-6 SE (4) - At Atlantic, on State Highway 83 bridge over East Nishnabotna River, on curb at left upstream end of bridge, an Iowa Highway Commission bench mark.

Elev. 1152.91 feet.

7636-6 SE (5) - At west edge of Atlantic, on 6th Street bridge over East Nishnabotna River, on top of left upstream wingpost, a chiseled square. Elev. 1154.18 feet.

7636-6 SE (6) - (Reference point) At west edge of Atlantic, on 6th Street bridge over East Nishnabotna River, on downstream guardrail, near 21st guardrail post from left end of bridge, a filed arrow.

Elev. 1154.39 feet.

7637-14 SE (1) - About 3 miles southwest of Atlantic, on county road bridge over East Nishnabotna River, on upstream curb, at right end of bridge, a chiseled square.

Elev. 1140.00 feet.

7637-23 NE (1) - About 3 miles southwest of Atlantic, on county road bridge over East Nishnabotna River, on downstream curb, at right end of bridge, a chiseled square.

E1ev. 1140.02 feet.

7637-23 NE (2) - About 3 miles southwest of Atlantic, on county road bridge over East Nishnabotna River, on downstream curb, between 2 nd and $3 \mathrm{rd}$ vertical truss members from right end of bridge, a chiseled square.

Elev. 1141.13 feet.

7637-26 SW (1) About 2.5 miles north of Lewis, on county road bridge over East Nishnabotna River, on inside of left upstream bridge abutment, a chiseled square. Elev. 1131.76 feet.

7637-26 SW (2) About 2.5 miles north of Lewis, on county road bridge over East Nishnabotna River, on left upstream corner of right upstream bridge abutment, a chiseled square. Elev. 1134.12 feet.

7639-18 NE (1) - About 0.5 mile west of Hancock, on county road G30 bridge over West Nishnabotna River, on downstream curb at right end of bridge, a chiseled square.

Elev. 1115.76 feet. 
7639-18 NE (2) - About 0.5 mile west of Hancock, on county road G30 bridge over West Nishnabotna River, on downstream curb at left end of bridge, painted "UE 1116.2" from Avoca Quad.84, line 7 in 1962, a chiseled square. Elev. 1115.92 feet.

7639-18 SE (1) - About 0.8 mile south of the Hancock school in Hancock along U.S. Highway 59, 137 feet south of the south edge of railroad bridge, 15 feet northeast of centerline of a field road entrance, 1.5 feet northwest of southeast right of way fence, a standard tablet stampled "J 1471949 " set in top of a concrete post.

Elev. 1108.397 feet.

7639-18 SW (1) - About 1 mile southwest of Hancock, on railroad bridge over West Nishnabotna River, on left upstream wingpost, a chiseled square. Elev. 1127.34 feet.

7639-18 SW (2) - (Reference point) About 1 mile southwest of Hancock, on railroad bridge over West Nishnabotna River, at center of bridge on downstream side, on top of a bolt head which is on top of a steel crossbar support, a chiseled arrow.

Elev. 1129.07 feet.

7736-1 SE (1) - About 0.8 mile east of intersection of Interstate 80 and U.S. Highway 71 , on downstream Interstate 80 bridge over East Nishnabotna River, on downstream abutment at right end of bridge, an Iowa Highway Commission bench mark.

Elev. 1194.95 feet.

7736-1 SE (2) - (Reference point) About 0.8 mile east of intersection of Interstate 80 and U.S. Highway 71, on downstream Interstate 80 bridge over East Nishnabotna River, on downstream curb, near 19th guardrail post from right end of bridge, a chiseled square.

E1ev. 1195.24 feet.

7736-12 NW (1) - About 0.5 mile downstream of Interstate 80, on county road G16 bridge over East Nishnabotna River, on downstream curb at right end of bridge, top of bolt. (Unable to locate $5 / 31 / 89$. )

Elev. 1191.47 feet.

7736-12 NW (2) - (Reference point) About 0.5 mile downstream of Interstate 80 , on county road G16 bridge over East Nishnabotna River, on 14th guardrail post from right downstream end of bridge, a filed arrow. Elev. 1193.98 feet.

7736-12 NW (3) - About 0.5 mile downstream of Interstate 80 , on county road G16 bridge over East Nishnabotna River, on downstream guardrail, at right of 3 rd guardrail post from right end of bridge, top of guardrail.

Elev. 1193.62 feet.

7736-12 NW (4) - About 0.5 mile downstream of Interstate 80, on county road G16 bridge over East Nishnabotna River, behind wingwall at right downstream end of bridge, a chiseled square.

(Elevation to be surveyed.) 
7736-13 SW (1) - About 2.3 miles south of Interstate 80, on U.S. Highway 71 bridge over East Nishnabotna River, on right upstream abutment, a chiseled cross.

Elev. 1184.92 feet.

7736-13 NW (1) - About 2 miles south of Interstate 80 , on county road bridge over East Nishnabotna River, on right downstream bridge seat, a chiseled cross on bolt. Elev. 1182.09 feet.

7736-13 NW (2) - (Reference point) About 2 miles south of Interstate 80, on county road bridge over East Nishnabotna River, on 3 rd vertical truss member from right downstream end of bridge, a chiseled notch at guardrail height. (Unable to locate 5/31/89.) Elev. 1188.02 feet.

7736-13 NW (3)- About 2 miles south of Interstate 80, on county road bridge over East Nishnabotna River, under bridge, in pile at end of right truss, a nail and disk.

Elev. 1177.83 feet.

7736-13 NW (4) - About 2 miles south of Interstate 80, on county road bridge over East Nishnabotna River, on 3 rd guardrail post from left downstream end of bridge, a filed arrow. $\quad$ Elev. 1187.79 feet.

7736-14 SE (1) - (Reference point) About 2.3 miles south of Interstate 80 , on U.S. Highway 71 bridge over East Nishnabotna River, on downstream curb, at 19 th guardrail post from right end of bridge, a chiseled arrow. Elev. 1185.08 feet.

7736-27 SW (1) - About 1.5 miles northeast of Atlantic, on county road bridge over East Nishnabotna River, at left end of downstream side of bridge truss, a chiseled cross on bolt. Elev. 1164.62 feet.

7736-27 SW (2) - (Reference point) About 1.5 miles northeast of Atlantic, at county road bridge over East Nishnabotna River, on center vertical truss member on downstream side of bridge, a filed mark.

Elev. 1169.68 feet.

7736-27 SW (3) - (Reference point) About 1.5 miles northeast of Atlantic, on county road bridge over East Nishnabotna River, on downstream low-steel, at 5 th vertical truss member from left end of bridge, a filed arrow.

Elev. 1164.70 feet.

7736-28 SW (1) - About 1.5 miles north of Atlantic, on county road over East Nishnabotna River, on bridge truss seat at left downstream end of bridge, a chiseled cross on bolt.

Elev. 1158.92 feet.

7736-28 SW (2) - (Reference point) About 1.5 miles north of Alantic, on county road over East Nishnabotna River, on downstream side of bridge, on left leg of middle cross member of bridge truss, a filed mark at guardrail height.

Elev. 1164.17 feet.

7736-32 NE (1)- (Reference point) About 1.2 miles north of Atlantic, on county road N16 bridge over East Nishnabotna River, on downstream guardrail post at center of bridge, a chiseled arrow.

Elev. 1163.41 feet. 
7736-32 SW (1) - About 0.5 mile northwest of Atlantic, on county road bridge over East Nishnabotna River, on bridge seat at left downstream end of bridge truss, a filed cross on bolt. Elev. 1149.76 feet.

7736-32 SW (2) - (Reference point) About 0.5 mile northwest of Atlantic, on county road bridge over East Nishnabotna River, on downstream guardrail, between center vertical truss members, a chiseled arrow. Elev. 1155.42 feet.

7736-33 NW (1) - About 1.2 miles north of Atlantic, on county road N16 bridge over East Nishnabotna River, on top of left upstream wingwall, a chiseled square.

Elev. 1161.05 feet.

7739-5 SE (1) - About 1.5 miles north of Avoca, on downstream Interstate 80 bridge over West Nishnabotna River, on right downstream wingwall behind wingpost, an Iowa Highway Commission bench mark.

Elev. 1147.87 feet.

7739-5 SE (2) - (Reference point) About 1.5 miles north of Avoca, on downstream Interstate 80 bridge over West Nishnabotna River, on downstream curb, between 16 th and 17 th guardrail posts from right end of bridge, a chiseled square.

Elev. 1148.89 feet.

7739-5 NW (1) - About 2 miles northwest of Avoca, at a concrete culvert under railroad, about 300 feet north of center of Interstate 80 overpass, 39 feet south of centerline of east-west gravel road, 16 feet south of center of railroad bridge 88, on east end of culvert headwal1, a standard disk stamped "T 146 RESET 1964".

Elev. 1158.296 feet.

7739-16 NW (1) - At Avoca, about 0.2 mile west along railroad from station in Avoca, at a road crossing, 148 feet south of south rail of main track, 52 feet east of centerline of Pine street, 65 feet southeast of a railroad crossing sign post, 1.1 feet north of a fence, 1.2 feet east of a reference post, a standard tablet stamped "R 1461949 " set in top of a concrete post.

Elev. 1137.681 feet.

7739-17 NE (1) - About 1 mile west of Avoca, on State Highway 83 bridge over West Nishnabotna River, on right downstream wingpost, an Iowa Highway Commission bench mark.

Elev. 1142.02 feet.

7739-17 NE (2) - (Reference point) About 1 mile west of Avoca, on State Highway 83 bridge over West Nishnabotna River, on 14 th guardrail post from right downstream end of bridge, a chiseled arrow.

Elev. 1140.07 feet.

7739-17 SE (1) - About 1 mile west of Avoca, on Union Street bridge over West Nishnabotna River, at left downstream corner of bridge, top of bolt painted "UE". (Bridge abandoned 6/13/89.) Elev. 1131.20 feet.

7739-17 SE (2) - (Reference point) About 1 mile west of Avoca, on Union Street bridge over West Nishnabotna River, on downstream side of bridge, on 3 rd vertical truss member from left end of bridge, a filed notch. (Bridge abandoned 6/13/89.) Elev. 1135.68 feet. 
7835-SW (1) - At Exira, on U.S. Highway 71 bridge over East Nishnabotna River, on left downstream wingwall curb, a standard National Geodetic Survey brass tablet. Elevation obtained from Iowa Dept. of Transportation.

Elev. 1233.506 feet.

7835-4 SW (2) - (Reference point) At Exira, on U.S. Highway 71 bridge over East Nishnabotna River, on downstream concrete guardrail, 159 feet from brass tablet on left downstream end of bridge, a chiseled square. Elevation established from 7835-4 SW (1). Elev. 1236.40 feet.

7835-4 NW (1) - At Exira, on county road F58 bridge over East Nishnabotna River, on downstream abutment at right end of bridge, a chiseled square.

Elev. 1228.13 feet.

7835-4 NW (2) - (Reference point) At Exira, on county road F58 bridge over East Nishnabotna River, on 13 th guardrail post from right downstream end of bridge, a filed arrow. Elev. 1230.07 feet.

7835-19 NE (1) - About 0.5 mile east of Brayton, on county road bridge over East Nishnabotna River, on downstream wingwall at right end of bridge, a chiseled square. (Unable to locate 5/31/89.) Elev. 1214.69 feet.

7835-19 NE (2) - (Reference point) About 0.5 mile east of Brayton, on county road bridge over East Nishnabotna River, on 17 th guardrail post from right downstream end of bridge, a filed arrow.

Elev. 1216.77 feet.

7835-19 NE (3) - (Reference point) About 0.5 mile east of Brayton, on county road bridge over East Nishnabotna River, on 20th guardrail post from right downstream end of bridge, a filed cross.

(Elevation to be surveyed.)

7835-19 NE (4) - About 0.5 mile east of Brayton, on county road bridge over East Nishnabotna River, on right downstream wingwall, a chiseled cross.

(Elevation to be surveyed.)

7835-19 NE (5) - About 0.5 mile east of Brayton, on county road bridge over East Nishnabotna River, at extreme end of right downstream wingwall, a deeply chiseled cross.

(Elevation to be surveyed.)

7835-19 SW (1) - About 0.4 mile south along railroad from intersection with East Main Street in Brayton, on railroad bridge marked 451.7, at upstream side of bridge at right abutment, a pole spike and collar in top beam.

Elev. 1206.90 feet.

7835-29 NW (1) - About 1 mile southeast of Brayton, near north sixteenth corner between sections 29 and 30,32 feet north and 510 feet east of T-road east, 172 feet east of fence, 2 feet west of power pole, a standard disk stamped "11 LB 1956" set in a concrete post.

Elev. 1356.660 feet. 
7836-24 SE (1) - About 0.2 mile south of Brayton, on railroad bridge over creek, at upstream side of bridge on right abutment, a pole spike and collar.

Elev. 1204.66 feet.

7836-25 SW (1) - About 1.8 mile south along railroad from intersection with East Main Street in Brayton, on railroad bridge marked 450.3, at upstream side of bridge at right abutment, a pole spike and collar in top beam.

Elev. 1195.77 feet.

7836-36 SE (1) - About 2 miles south of Brayton and 1 mile north of Interstate 80 , on county road bridge over East Nishnabotna River, on end of left downstream wingwall, a chiseled square.

(Elevation to be surveyed.)

7836-36 SE (2) - (Reference point) About 2 miles south of Brayton and 1 mile north of Interstate 80 , on county road bridge over East Nishnabotna River, on 17 th guardrail post from right downstream end of bridge, a filed arrow.

(Elevation to be surveyed.)

7836-36 SW (1) - Near county line about 2.5 miles south of Brayton, at pipeline crossing over East Nishnabotna River, most westerly pipeline support, filed cross on center I-beam. Elev. 1193.57 feet.

7839-4 SE (1) - At the Harlan Municipal airport, about 4 miles south of Harlan, 2.1 feet north of northwest corner of main hanger and office, 3 feet northeast of the northeast corner of concrete sidewalk on west side of hangar, a standard tablet stamped "L 1501949 " set in a concrete post.

Elev. 1192.895 feet.

7839-9 SE (1) - About 5 miles south of Harlan, on U.S. Highway 59 bridge over West Nishnabotna River, on right downstream wingpost, a chiseled square. Elev. 1175.06 feet.

7839-9 SE (2) - (Reference point) About 5 miles south of Harlan, on U.S. Highway 59 bridge over West Nishnabotna River, on 30 th guardrail post from right downstream end of bridge, a chiseled arrow.

E1ev. 1172.74 feet.

7839-9 SE (3) - About 5 miles south of Harlan, on U.S. Highway 59 bridge over West Nishnabotna River, on upstream curb at left end of bridge, a chiseled cross. Elevation obtained from Iowa Dept. of Transportation.

Elev. 1172.67 feet.

7839-10 NW (1) - At Corley, on county road F58 bridge over West Nishnabotna River, on right downstream wingpost, a chiseled square. (Unable to locate 6/9/89.)

Elev. 1177.74 feet.

7839-10 NW (2) - (Reference point) At Corley, on county road F58 bridge over West Nishnabotna River, on 15th guardrail post from right downstream end of bridge, a filed arrow.

Elev. 1177.94 feet.

7839-10 NW (3) - At Corley, on county road F58 bridge over West Nishnabotna River, on downstream curb, behind wingwall at right end of bridge, a chiseled square.

(Elevation to be surveyed.) 
7839-28 SW (1) - About 3 miles north of Avoca along U.S. Highway 59, at a road crossing, 89 feet north of center of farm driveway, 80 feet west of center of U.S. Highway 59, 1 foot north of a witness post, a standard tablet stamped "83-19 RESET 1970" set in a concrete post.

Elev. 1161.886 feet.

7839-32 SE (1) - About 2 miles north of Avoca, on county road F66 bridge over West Nishnabotna River, on downstream concrete guardrail, at right end of bridge, a chiseled square. Elevation established from 7839-32 SE (3).

Elev. 1153.50 feet.

7839-32 SE (2) - (Reference point) About 2 miles north of Avoca, on county road F66 bridge over West Nishnabotna River, on downstream concrete guardrail, 151 feet from right end of bridge, a chiseled square. Elevation established from 7839-32 SE (3). Elev. 1152.70 feet.

7839-32 SE (3) - About 2 miles north of Avoca, at a grain bin about 600 feet east of county road F66 bridge over West Nishnabotna River, on southeast corner of cement platform, a chiseled cross. Elevation obtained from Shelby County Engineer. Elev. 1143.01 feet.

7839-33 NW (1) - About 2 miles north of Avoca, at northwest corner of intersection of U.S. Highway 59 and county road F66, on north side of pole 2 feet above ground, a nail and disk. Elev. 1146.31 feet.

7935-15 SE (1) - About 0.5 mile south of Hamlin, on U.S. Highway 71 bridge over East Nishnabotna River, on upstream abutment at left end of bridge, a chiseled square. (Unable to locate 5/30/89.)

Elev. 1258.47 feet.

7935-15 SE (2) - (Reference point) About 0.5 mile south of Hamlin, on U.S. Highway 71 bridge over East Nishnabotna River, on downstream curb, at 13 th guardrail post from left end of bridge, a chiseled arrow.

Elev. 1258.57 feet.

7935-15 SE (3) - About 0.5 mile south of Hamlin, on U.S. Highway 71 bridge over East Nishnabotna River, on upstream abutment at left end of bridge, a chiseled cross.

(Elevation to be surveyed.)

7935-15 SE (4) - (Reference point) About 0.5 mile south of Hamlin, on U.S. Highway 71 bridge over East Nishnabotna River, on downstream curb, at 16 th guardrail post from left end of bridge, a chiseled cross.

(Elevation to be surveyed.)

7935-22 NE (1) - About 1.5 miles south of Hamlin, on county road bridge over East Nishnabotna River, on right end of truss on downstream side of bridge, a filed cross. (Unable to locate 5/30/89.)

E1ev. 1251.48 feet.

7935-22 NE (2) - (Reference point) About 1.5 miles south of Hamlin, on county road bridge over East Nishnabotna River, on downstream guardrail, between 2 nd and 3 rd vertical truss members from right end of bridge, a filed arrow.

Elev. 1255.14 feet. 
7935-22 NE (3) - About 1.5 miles south of Hamlin, on county road bridge over East Nishnabotna River, on right end of bridge truss 0.9 feet below downstream bridge deck, a filed arrow.

(Elevation to be surveyed.)

7935-27 NE (1) - About 2.5 miles north of Exira, on county road bridge over East Nishnabotna River, on top of downstream wingwall at left end of bridge, a chiseled square. Elevation established from 7935-27 NE (3).

Elev. 1249.77 feet.

7935-27 NE (2) - (Reference point) About 2.5 miles north of Exira, on county road bridge over East Nishnabotna River, at center of downstream concrete guardrail, a chiseled cross. Elevation established from 7935-27 NE (3).

Elev. 1249.90 feet.

7935-27 NE (3) - About 2.5 miles north of Exira, about 100 feet east of county road bridge over East Nishnabotna River, a spike in corner post of fence. Elevation obtained from Audubon County Engineer.

Elev. 1246.52 feet.

7935-34 NE (1) - About 1.5 miles north of Exira, on county road bridge over East Nishnabotna River, at right end of truss on downstream side of bridge, a filed cross on bolt.

Elev. 1241.16 feet.

7935-34 NE (2) - (Reference point) About 1.5 miles north of Exira, on county road bridge over East Nishnabotna River, on downstream guardrail at center of bridge, a filed arrow. Elev. 1244.78 feet.

7935-35 SW (1) - About 0.5 mile north of Exira, on county road bridge over East Nishnabotna River, at right end of truss on downstream side of bridge, on top of landward bolt, a filed cross. Elev. 1232.17 feet.

7935-35 SW (2) - (Reference point) About 0.5 mile north of Exira, on county road bridge over East Nishnabotna River, on downstream guardrail, between 1 st and 2 nd vertical truss members from right end of bridge, a filed arrow.

Elev. 1235.90 feet.

7938-4 SE (1) - About 2 miles northeast of Harlan, on county road bridge over West Nishnabotna River, on left upstream bridge seat, on 1 inch bolt head, a chiseled cross.

Elev. 1204.41 feet.

7938-4 SE (2) - (Reference point) About 2 miles northeast of Harlan, on county road bridge over West Nishnabotna River, on downstream guardrail, at 3 rd vertical truss member from left end of bridge, a filed arrow.

Elev. 1210.70 feet.

7938-8 SE (1) - About 1 mile northeast of Harlan, on county road bridge over West Nishnabotna River, on left downstream most landward bolt head, a chiseled cross.

Elev. 1200.16 feet. 
7938-8 SE (2) - (Reference point) About 1 mile northeast of Harlan, on county road bridge over West Nishnabotna River, on downstream guardrail, at 4 th vertical truss member from left end of bridge, a filed arrow.

Elev. 1204.07 feet.

7938-8 SW (1) - At northeast edge of Harlan, on bridge over West Fork West Nishnabotna River, on top of right downstream railing, near bridge span, a chiseled cross.

Elev. 1202.72 feet.

7938-19 NE (1) - At Harlan, about 1 mile east along State Highway 44 from junction with U.S. Highway 59 , on a steel power line tower, on northwest concrete base, a standard disk stamped "V 1501949 ".

Elev. 1191.458 feet.

7938-19 NE (2) - At Harlan, on State Highway 44 bridge over West Nishnabotna River, on right upstream concrete abutment, an Iowa Highway Commission bench mark. Elevation obtained from Iowa Dept. of Transportation.

Elev. 1200.07 feet.

7938-19 NE (3) - (Reference point) At Harlan, on State Highway 44 bridge over West Nishnabotna River, on downstream concrete guardrail, near light base bolts, a chiseled square. Elevation established from 7938-19 NE (2).

Elev. 1200.13 feet.

8038-3 SW (1) - About 2 miles north of Kirkman, on county road bridge over West Nishnabotna River, at left upstream corner of bridge, at upstream landward corner, on top of 0.5 inch bolt at left end of truss, a filed cross.

Elev. 1240.59 feet.

8038-10 NW (1) - (Reference point) About 2 miles north of Kirkman, on county road bridge over West Nishnabotna River, on 2 nd vertical truss member from right downstream end of bridge, a filed mark.

Elev. 1244.60 feet.

8038-22 NE (1) - About 0.3 mile northeast of Kirkman, on county road F32 bridge over West Nishnabotna River, on right downstream wingwal1, a chiseled square.

(Elevation to be surveyed.)

8038-22 NE (2) - (Reference point) About 0.3 mile northeast of Kirkman, on county road F32 bridge over West Nishnabotna River, on right half of downstream concrete guardrail, a chiseled square.

(Elevation to be surveyed.)

8038-22 NE (3) - At east edge of Kirkman, on county road bridge over West Nishnabotna River, at left downstream end of truss, on top of pile cap, a chiseled cross on top of 1 inch bolt head. (Unable to locate 6/8/89.) Elev. 1226.48 feet.

8038-22 NE (4) - (Reference point) At east edge of Kirkman, on county road bridge over West Nishnabotna River, on 2 nd vertical truss member from right downstream end of bridge, a filed mark. Elev. 1233.01 feet. 
8038-33 NE (1) - About 1.5 miles south of Kirkman, on county road bridge over West Nishnabotna River, on downstream landward corner of left downstream steel pile cap, a chiseled square. Elev. 1216.40 feet.

8038-33 NE (2) - (Reference point) About 1.5 miles south of Kirkman, on county road bridge over West Nishnabotna River, on 4th vertical truss member from left downstream end of bridge, a filed mark.

Elev. 1222.30 feet.

8038-33 NE (3) - (Reference point) About 1.5 miles south of Kirkman, on county road bridge over West Nishnabotna River, on downstream guardrail, between 2nd and 3rd vertical truss members from left end of bridge, a filed arrow.

Elev. 1222.20 feet.

8137-2 NE (1) - (Reference point) About 7 miles northeast of Irwin, at county-1ine road bridge over West Nishnabotna River, on 2nd vertical truss member from left downstream end of bridge, a filed mark. Also see 8237-35 SE (1).

Elev. 1309.40 feet.

8137-2 SW (1) - About 0.5 mile south of Botna, 0.4 mile south of a church at Botna, 33 feet east of centerline of county road, 81 feet northeast of the third power line pole north of intersection, 3 feet north of third telephone pole north of intersection, 0.8 feet west of a fence, 1.3 feet south of a reference post, a standard tablet stamped

"Q 151 1949" set in top of a concrete post. Elev. 1344.558 feet.

8137-3 NE (1) - About 6 miles northeast of Irwin, on county road M56 bridge over West Nishnabotna River, on curb at right downstream end of bridge, a chiseled square.

Elev. 1300.50 feet.

8137-3 NE (2) - (Reference point) About 6 miles northeast of Irwin, on county road M56 bridge over West Nishnabotna River, on 13th guardrail post from right downstream end of bridge, a filed arrow.

Elev. 1302.34 feet.

8137-3 NE (3) - (Reference point) About 6 miles northeast of Irwin, on county road M56 bridge over West Nishnabotna River, on 8 th guardrail post from left downstream end of bridge, a filed arrow.

Elev. 1302.32 feet.

8137-3 SW (1) - About 1 mile southwest of Botna, on coutny road F16 bridge over West Nishnabotna River, on steel pile cap at left upstream end of bridge, a chiseled cross on a rivet. (Unable to locate $6 / 8 / 89$.)

Elev. 1294.60 feet.

8137-4 SE (1) - About 1 mile west of Botna, at southeast corner of section 4, near northeast corner of a schoolyard, 204 feet north of centerline of county road F16, 62 feet west of centerline of north-south county road, 30 feet west of a corner fence post, 1.2 feet south of a fence, a standard tablet stamped "P 151 1949" set in a concrete post.

Elev. 1339.961 feet. 
8137-10 NW (1) - (Reference point) About 1 mile southwest of Botna, on county road F16 bridge over West Nishnabotna River, on downstream side of bridge, on 2nd vertical truss member from left downstream end of bridge, on right side of truss member, a filed notch.

Elev. 1300.29 feet.

8137-16 NE (1) - About 4 miles northeast of Irwin, on county road bridge over West Nishnabotna River, on top of steel pile cap at right downstream end of truss, a chiseled square. (Bridge abandoned 6/8/89.)

Elev. 1286.12 feet.

8137-16 NE (2) - (Reference point) About 4 miles northeast of Irwin, on county road bridge over West Nishnabotna River, on center downstream bridge truss member, on right side of truss member, a filed mark. (Bridge abandoned 6/8/89.) Elev. 1290.58 feet.

8137-21 NE (1) - About 3 miles northeast of Irwin, on county road bridge over West Nishnabotna River, on downstream side of bridge, 3 feet left of right end of center span, on horizontal I-beam support, a chiseled cross.

Elev. 1284.82 feet.

8137-21 NE (2) - (Reference point) About 3 miles northeast of Irwin, on county road bridge over West Nishnabotna River, on downstream guardrail, 4 inches left of 5 th guardrail post from right end of bridge, a filed arrow.

Elev. 1289.44 feet.

8137-21 NE (3) - (Reference point) About 3 miles northeast of Irwin, on county road bridge over West Nishnabotna River, on 3 rd guardrail post from left downstream end of bridge, a chiseled arrow.

Elev. 1289.38 feet.

8137-29 SW (1) - (Reference point) About 1 mile northeast of Irwin, on county road bridge over West Nishnabotna River, on 2nd bridge truss member from left downstream end of bridge, a filed notch. (Bridge abandoned 6/8/89.)

Elev. 1270.71 feet.

8137-29 NW (1) - About 1 mile northeast of Irwin, on county road bridge over West Nishnabotna River, on pile cap at left upstream end of bridge, a chiseled cross on rivet. (Bridge abandoned 6/8/89.)

Elev. 1266.45 feet.

8137-31 NE (1) - At east edge of Irwin, on county road F24 bridge over West Nishnabotna River, on curb at right downstream abutment, a 2 inch brass cap.

Elev. 1264.92 feet.

8137-31 NE (2) - (Reference point) At east edge of Irwin, on county road F24 bridge over West Nishnabotna River, on 2 nd guardrail post from right of left downstream pier, a filed arrow. (Unable to locate $6 / 8 / 89$.

Elev. 1266.89 feet.

8137-31 NE (3) - (Reference point) At east edge of Irwin, on county road F24 bridge over West Nishnabotna River, on 3rd guardrail post from left downstream pier, a filed arrow. Elev. 1267.00 feet. 
8138-25 SE (1) - At Irwin, at northwest corner of intersection of county road M47 and county road F24, 52 feet north of centerline of county road F24, 43 feet west of centerline of county road M47, 10.4 feet west of west edge sidewalk, 3.1 feet southwest of corner fence post, 1.6 feet south of a fence, a standard tablet stamped "L 151 1949" set in a concrete post.

Elev. 1263.793 feet.

8138-36 SE (1) - At south edge of Irwin, on county road M47 bridge over West Nishnabotna River, on curb of right downstream abutment, a standard tablet stamped USC\&GS.

Elev. 1262.02 feet.

8138-36 SE (2) - (Reference point) At south edge of Irwin, on county road M47 bridge over West Nishnabotna River, on downstream guardrail at center of bridge, a filed arrow. Elev. 1263.79 feet.

8236-19 NE (1) - About 0.5 mile south of Manning, on county road M66 bridge over West Nishnabotna River, on top of right downstream wingpost, a chiseled square.

Elev. 1324.97 feet.

8236-19 NE (2) - (Reference point) About 0.5 mile south of Manning, on county road M66 bridge over West Nishnabotna River, on 6 th guardrail post from left downstream end of bridge, a chiseled arrow.

Elev. 1325.10 feet.

8236-20 SW (1) - At the Manning airport about 1 mile southwest of Manning, 79 feet north of the northeast corner of the south hanger, 6.8 feet east of the southeast corner of the main office, 9.0 feet south of the southeast corner of a gas pump, 0.5 foot east of the southeast corner of a concrete slab, a standard tablet stamped "W 1511949 " set in top of a concrete post. Elev. 1315.48 feet.

8236-20 NW (1) - At Manning, on State Highway 141 bridge over West Nishnabotna River, on right downstream wingwa11, an Iowa Highway Commission bench mark. E1ev. 1326.33 feet.

8236-20 NW (2) - (Reference point) At Manning, on State Highway 141 bridge over West Nishnabotna River, on downstream curb, at 7 th guardrail post from right end of bridge, a chiseled arrow. (Unable to locate 6/8/89.)

Elev. 1326.98 feet.

8236-20 NW(3) - (Reference point) At Manning, on State Highway 141 bridge over West Nishnabotna River, on downstream curb, near 7 th guardrail post from right end of bridge, a chiseled square.

E1ev. 1326.96 feet.

8236-31 NW (1) - About 2 miles southwest of Manning, on east-west county road bridge over West Nishnabotna River, on top of left downstream wingpost, a chiseled square.

Elev. 1316.80 feet.

8236-31 NW (2) - (Reference point) About 2 miles southwest of Manning, on east-west county road bridge over West Nishnabotna River, on 7 th guardrail post from right downstream side of bridge, a filed arrow.

Elev. 1316.74 feet. 
8237-35 SE (1) - About 7 miles northeast of Irwin, at county-1ine road bridge over West Nishnabotna River, on right upstream bridge seat, a chiseled cross on rivet. Also see $8137-2 \mathrm{NE}(1)$.

Elev. 1305.60 feet.

8237-35 SE (2) - About 7.5 miles northeast of Irwin, on north-south county road M64 bridge over West Nishnabotna River, on top of right downstream wingpost, a chiseled square. Elev. 1310.19 feet.

8237-35 SE (3) - (Reference point) About 7.5 miles northeast of Irwin, on north-south county road M64 bridge over West Nishnabotna River, on 9 th guardrail post from right downstream end of bridge, a filed arrow. Elev. 1310.10 feet.

8237-36 NE (1) - About 3 miles southeast of Aspinwa11, on county-1ine road bridge over West Nishnabotna River, on top of right downstream wingpost, a chiseled square. Elev. 1315.17 feet.

8237-36 NE (2) - (Reference point) About 3 miles southeast of Aspinwall, on county-1ine road bridge over West Nishnabotna River, on downstream guardrail, at left of 9 th guardrail post from right end of bridge, a filed arrow. Elev. 1315.06 feet. 